Florida International University FIU Digital Commons

3-22-2013

\title{
Optimization of Wireless Power Transfer via Magnetic Resonance in Different Media
}

Olutola Jonah

Florida International, ojona001@fiu.edu

DOI: $10.25148 /$ etd.FI13042506

Follow this and additional works at: https:// digitalcommons.fiu.edu/etd

Part of the Electromagnetics and Photonics Commons, Other Electrical and Computer Engineering Commons, and the Power and Energy Commons

\section{Recommended Citation}

Jonah, Olutola, "Optimization of Wireless Power Transfer via Magnetic Resonance in Different Media" (2013). FIU Electronic Theses and Dissertations. 876.

https://digitalcommons.fiu.edu/etd/876

This work is brought to you for free and open access by the University Graduate School at FIU Digital Commons. It has been accepted for inclusion in FIU Electronic Theses and Dissertations by an authorized administrator of FIU Digital Commons. For more information, please contact dcc@fiu.edu. 


\section{FLORIDA INTERNATIONAL UNIVERSITY}

Miami, Florida

\section{OPTIMIZATION OF WIRELESS POWER TRANSFER VIA MAGNETIC RESONANCE IN DIFFERENT MEDIA}

A dissertation submitted in partial fulfillment of the

requirements for the degree of

DOCTOR OF PHILOSOPHY

in

ELECTRICAL ENGINEERING

by

Olutola Jonah

2013 
To: Dean Amir Mirmiran

College of Engineering and Computing

This dissertation, written by Olutola Jonah, and entitled Optimization of Wireless Power Transfer via Magnetic Resonance in Different Media, having been approved in respect to style and intellectual content, is referred to you for judgment.

We have read this dissertation and recommend that it be approved.

Osama A. Mohammed

Nezih Pala

Berrin Tansel

Stavros V. Georgakopoulos, Major Professor

Date of Defense: March 22, 2013

The dissertation of Olutola Jonah is approved.

Dean Amir Mirmiran College of Engineering and Computing

Dean Lakshmi N. Reddi University Graduate School

Florida International University, 2013 
C Copyright 2013 by Olutola Jonah

All rights reserved. 


\section{DEDICATION}

I dedicate this dissertation to the Almighty God for he is my All. And to my

lovely family, for their love, forbearance, understanding and cooperation, without which the completion of this work would not have been achievable. 


\section{ACKNOWLEDGMENTS}

I would like to convey my profound gratitude to my major professor, Dr. Stavros V. Georgakopoulos, for his outstanding guidance and support throughout my Ph.D. program at FIU. He provided me the favorable research conditions and constructive academic advice, with the latest research tools, which enabled the quality of my research and education. I am sincerely grateful for his assistance and patience during these four crucial years of my life. I am also appreciative of Dr. Osama A. Mohammed, Dr. Nezih Pala, and Dr. Berrin Tansel for serving on my dissertation defense committee and for their illuminating comments.

I am exceedingly thankful for my family who encouraged me during the research. Without my wife's love and understanding, it would have been difficult for me to conduct this research. I also deeply thank all the members of the FIU Electrical Engineering Department and special thanks to Maria Benicasa, Dr Shan Jiang, Dr. Charles Kamhoua, Yipeng Qu, Oscar Silveira, Arvind Merwaday, Shun Yao and Hao Hu.

Finally, I would like to thank FIU's Graduate School for awarding me a Dissertation Year Fellowship, which supported me in the last year of my Ph.D research. 


\title{
ABSTRACT OF THE DISSERTATION \\ OPTIMIZATION OF WIRELESS POWER TRANSFER VIA MAGNETIC \\ RESONANCE IN DIFFERENT MEDIA
}

\author{
by
}

Olutola Jonah

Florida International University, 2013

Miami, Florida

\section{Professor Stavros. V. Georgakopoulos, Major Professor}

A wide range of non-destructive testing (NDT) methods for the monitoring the health of concrete structure has been studied for several years. The recent rapid evolution of wireless sensor network (WSN) technologies has resulted in the development of sensing elements that can be embedded in concrete, to monitor the health of infrastructure, collect and report valuable related data. The monitoring system can potentially decrease the high installation time and reduce maintenance cost associated with wired monitoring systems. The monitoring sensors need to operate for a long period of time, but sensors batteries have a finite life span. Hence, novel wireless powering methods must be devised.

The optimization of wireless power transfer via Strongly Coupled Magnetic Resonance (SCMR) to sensors embedded in concrete is studied here. First, we analytically derive the optimal geometric parameters for transmission of power in the air. This specifically leads to the identification of the local and global optimization parameters and conditions, it was validated through electromagnetic simulations. Second, the optimum conditions were employed in the model for propagation of energy through plain and reinforced concrete at different humidity conditions, and frequencies with 
extended Debye's model. This analysis leads to the conclusion that SCMR can be used to efficiently power sensors in plain and reinforced concrete at different humidity levels and depth, also validated through electromagnetic simulations.

The optimization of wireless power transmission via SMCR to Wearable and Implantable Medical Device (WIMD) are also explored. The optimum conditions from the analytics were used in the model for propagation of energy through different human tissues. This analysis shows that SCMR can be used to efficiently transfer power to sensors in human tissue without overheating through electromagnetic simulations, as excessive power might result in overheating of the tissue.

Standard SCMR is sensitive to misalignment; both 2-loops and 3-loops SCMR with misalignment-insensitive performances are presented. The power transfer efficiencies above $50 \%$ was achieved over the complete misalignment range of $0^{\circ}-90^{\circ}$ and dramatically better than typical SCMR with efficiencies less than $10 \%$ in extreme misalignment topologies. 


\section{TABLE OF CONTENTS}

CHAPTER

PAGE

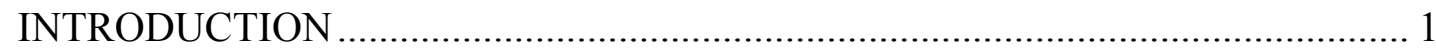

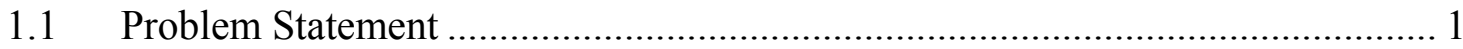

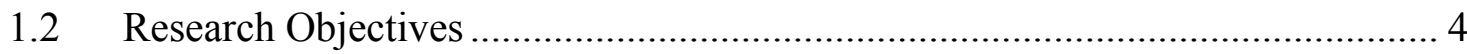

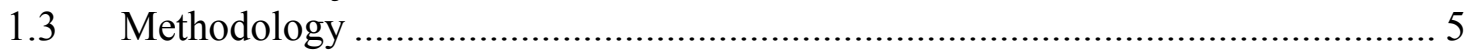

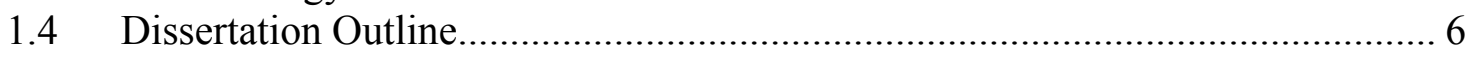

RELATED WORK

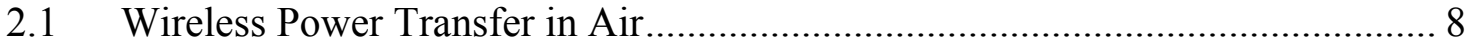

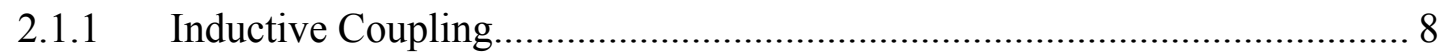

2.1.2 Electromagnetic Radiation.................................................................. 9

2.1.3 Strongly Coupled Magnetic Resonance (SCMR) ……………................... 9

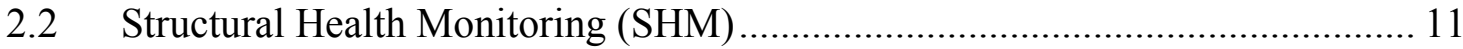

2.2.1 Wireless Sensor Networks …………………..................................... 13

2.2.2 Wireless Power Transmission for SHM ………........................................ 14

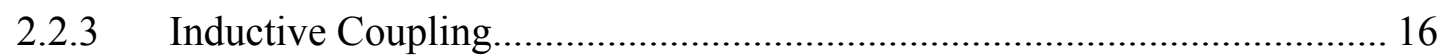

2.2.4 Electromagnetic Radiation .................................................................... 16

2.3 Wearable and Implantable Medical Devices (WIMD) ..................................... 17

2.3.1 Inductive Coupling............................................................................. 18

2.3.2 Strongly Coupled Magnetic Resonance (SCMR) ……………………....... 19

2.3.3 Electromagnetic (EM) Radiation …………….................................... 20

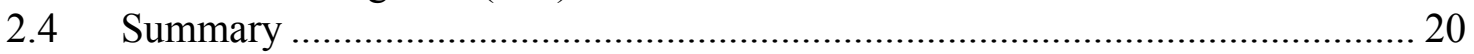

OPTIMIZATION FOR STRONGLY COUPLED MAGNETIC RESONANCE ..... 21

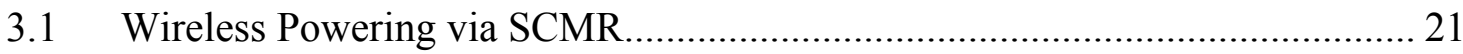

3.2 Optimal Loop and Helical Structures for SCMR .............................................. 23

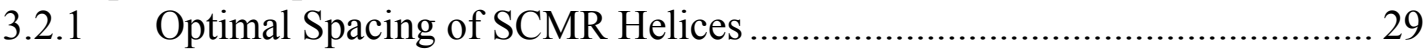

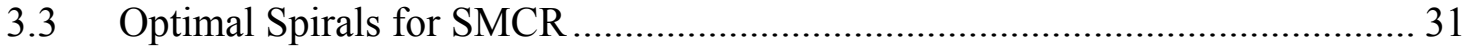

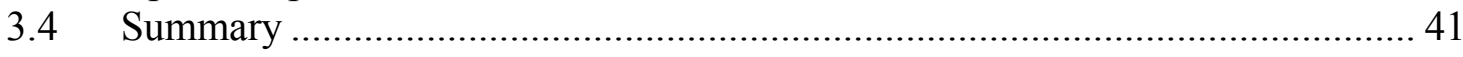

WIRELESS POWER TRANSFER IN CONCRETE STRUCTURES ……….......... 42

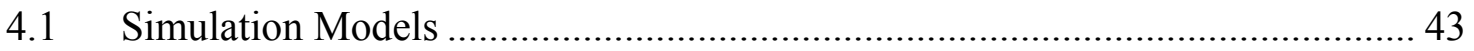

4.1.1 The Plain and Reinforced Concrete Models ............................................... 44

4.2 Optimization of Wireless Power Transfer in Concrete ...................................... 45

4.3 Wireless Power Transfer with Loops ................................................................. 47

4.3.1 Effects of varying TX loop's Placement Height.......................................... 51

4.3.2 Effects of varying RX loop's Placement Depth in Concrete ....................... 53

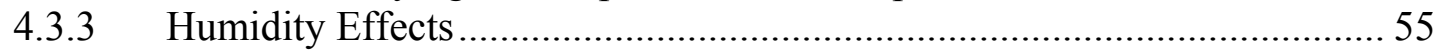

4.3.4 Effects of Reinforced Bars on Wireless Power Transfer ............................. 58

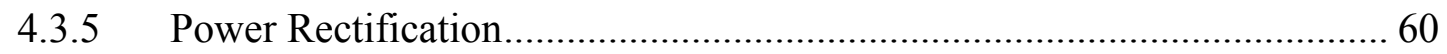

4.4 Wireless Power Transfer with Helix Structures .................................................... 61

4.5 Wireless Power Transfer with Spiral............................................................. 64 


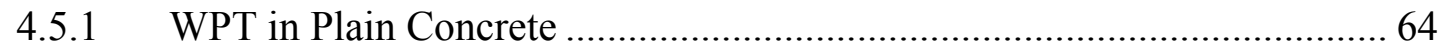

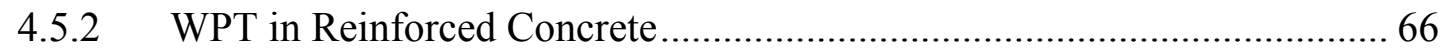

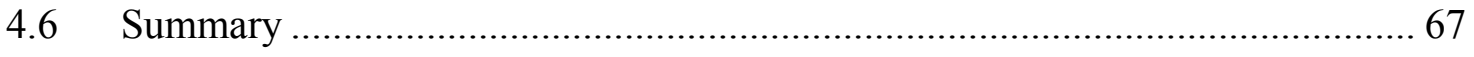

WIRELESS POWERING OF WEARABLE AND IMPLANTABLE MEDICAL

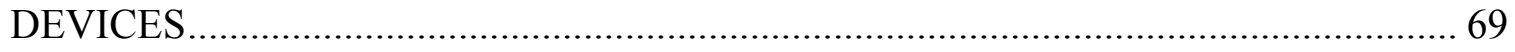

5.1 Powering of Wearable and Implantable Medical Devices ................................ 69

5.2 Wireless Power Transfer in Air-Tissue Interface via SCMR ............................ 70

5.3 Wireless Powering with Standard SCMR .................................................. 71

5.3.1 Wireless Power Transfer......................................................................... 71

5.3.2 Specific Absorption Rate (SAR) Analysis.................................................... 73

5.4 Conformal Strongly Coupled Magnetic Resonance (CSCMR) ……………..... 75

5.4.1 Conformal Structure for Wireless Power Transfer ...................................... 75

5.4.2 Conformal Bifilar Structure for Wireless Power Transfer.......................... 79

5.4.3 Specific Absorption Rate (SAR) Analysis................................................ 82

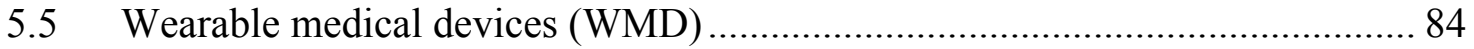

5.5.1 Wireless Powering of Wearable Devices.................................................... 85

5.5.2 Specific Absorption Rate (SAR) Analysis.................................................. 86

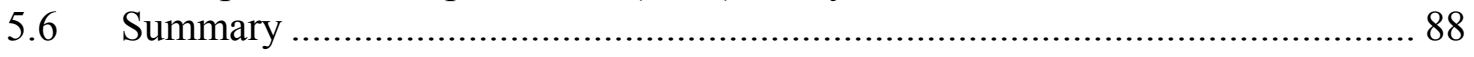

SIMULTANEOUS WIRELESS POWERING AND COMUNICATION IN RFID

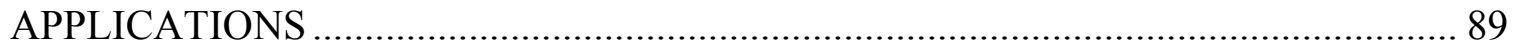

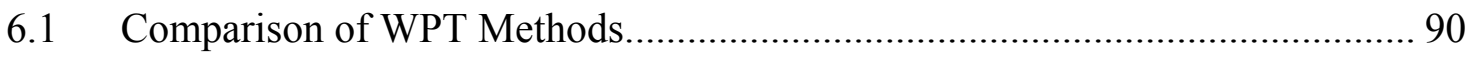

6.2 Wireless Powering with Resonant Inductive Coupling........................................ 92

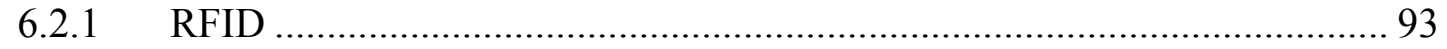

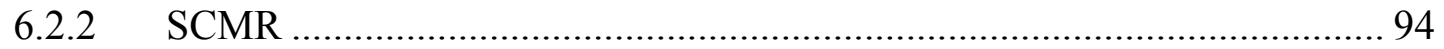

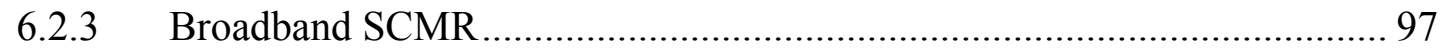

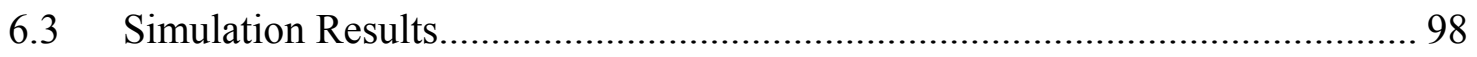

6.3.1 Modulation and data transfer (High Bandwidth) ..................................... 100

6.3.2 Modulation and data transfer (Low Bandwidth)...................................... 103

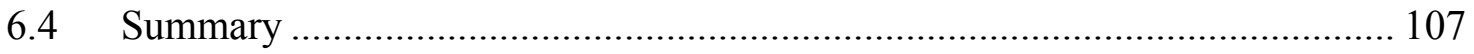

MISALIGNMENT INSENSITIVE SCMR SYSTEM ......................................... 108

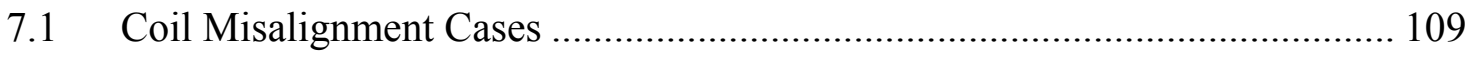

7.1.1 Angular elevation misalignment...................................................... 110

7.1.2 Angular azimuth misalignment.......................................................... 110

7.1.3 Lateral misalignment ……………………….................................... 110

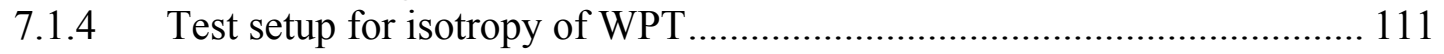

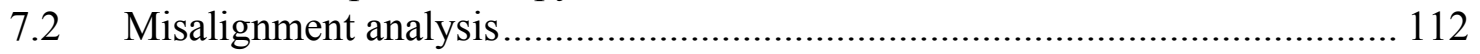

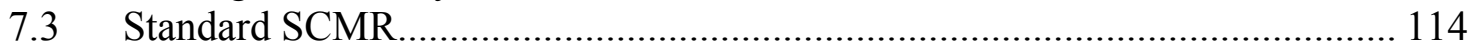

7.4 SCMR with four disconnected orthogonal loop pairs ........................................ 116

7.5 SCMR with two orthogonal and two parallel loops .......................................... 116

7.6 SCMR with embedded orthogonal loops ..................................................... 118

7.7 Misalignment Insensitive SCMR device (2- loop structure)............................ 120

7.8 Misalignment Insensitive SCMR (3- loop structure) ..................................... 122 


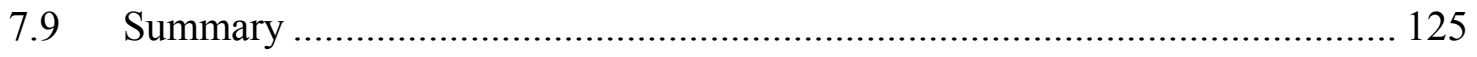

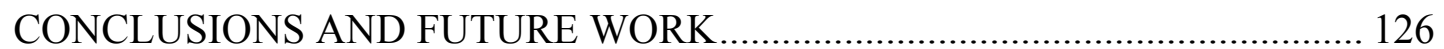

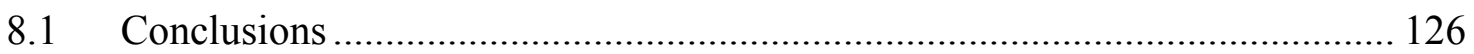

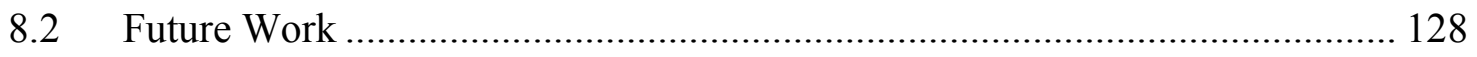

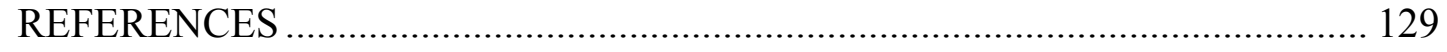

VITA................................................................... 144 


\section{LIST OF FIGURES}

FIGURE

PAGE

Figure 3.1: Schematic of an SCMR power transfer system in air.. .............................. 22

Figure 3.2: $Q_{\max }$ vs. electrical length of the loop.................................................... 27

Figure 3.3: Local maximum Q-factor $\left(Q_{\max }\right)$ vs. $\left(r_{c}\right)$ and optimal frequency $\left(f_{\max }\right) \ldots \ldots \ldots .27$

Figure 3.4: The efficiency of an SCMR system for different $r / r_{c}$ ratios...................... 29

Figure 3.5: The Q-factor and efficiency of helical SCMR with different spacing......... 31

Figure 3.6: Spiral schematic of an SCMR system with spirals in the air ...................... 32

Figure 3.7: The RLC representation of a spiral. .................................................... 32

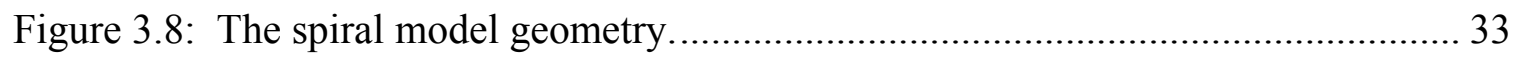

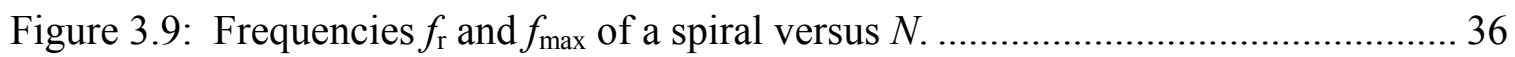

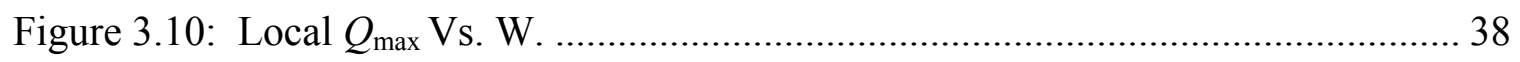

Figure 3.11: The efficiency of the SCMR system for $W=0.2,3.6$ and $9.0 \mathrm{~mm}$........... 39

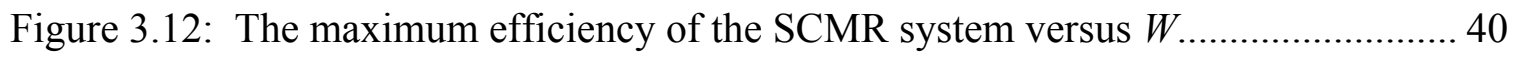

Figure 3.13: The frequency of maximum efficiency versus width of the spiral, $W . \ldots \ldots . .41$

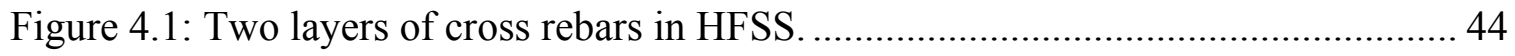

Figure 4.2: Reinforced concrete simulation setup in HFSS..................................... 45

Figure 4.3: An SCMR system for air-to-concrete transmission. ................................ 47

Figure 4.4: An SCMR system for air-to-concrete transmission. ................................50

Figure 4.5: Measurement setup of an SCMR system for air-to-concrete interface. ........ 51

Figure 4.6: Efficiency for various placement heights $(H)$ of the TX loop $(h=0.2 \%) \ldots 52$

Figure 4.7: Measured efficiency versus the placement height, $H$, of TX loop ................ 53

Figure 4.8: Efficiency for various placement depths, $D$, of the RX loop in concrete....... 54 
Figure 4.9: Measured efficiency versus the placement depth, $D$, of RX loop ................. 54

Figure 4.10: Efficiency vs. frequency in optimized SCMR designs............................ 57

Figure 4.11: Efficiency vs. distance in optimized SCMR designs $(H=10 \mathrm{~cm}) \ldots \ldots \ldots \ldots . . . .57$

Figure 4.12: An SCMR system inside concrete with parallel rebars............................. 59

Figure 4.13: An SCMR system inside concrete with parallel crossbars........................ 59

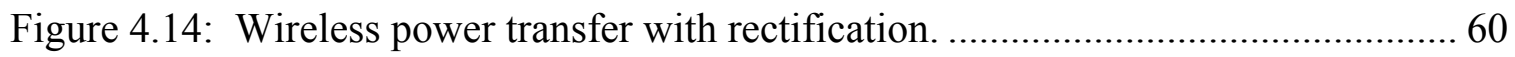

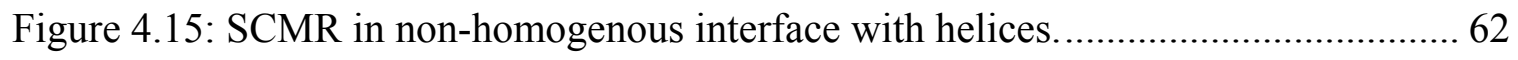

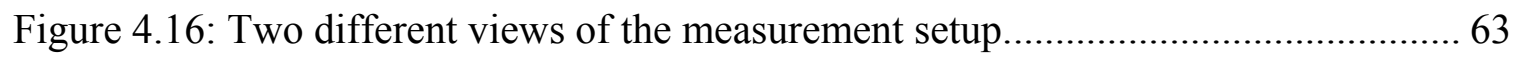

Figure 4.17: Efficiency of simulation and measurement results for $27.2 \mathrm{MHz} . \ldots \ldots \ldots \ldots . . . . .63$

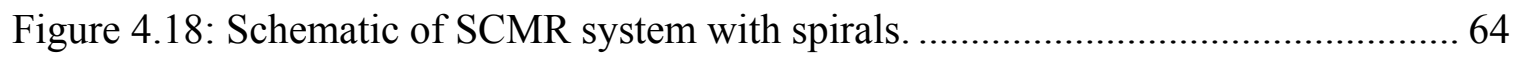

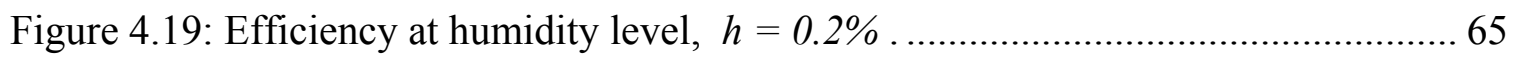

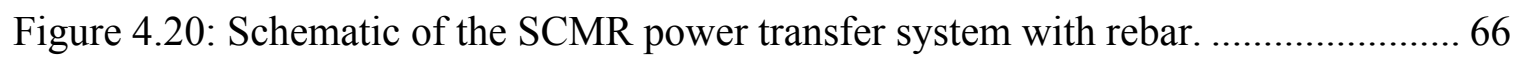

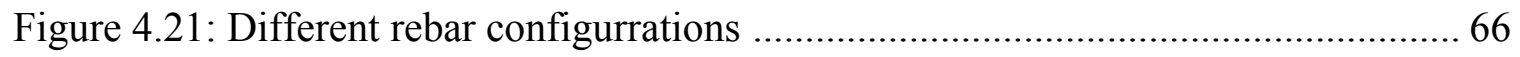

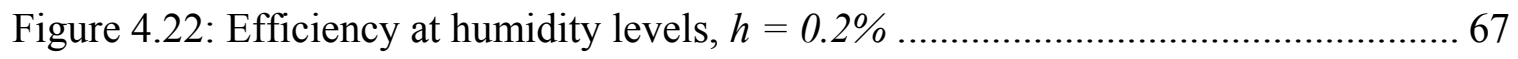

Figure 5.1: Schematic of SCMR power transfer system for tissue............................. 70

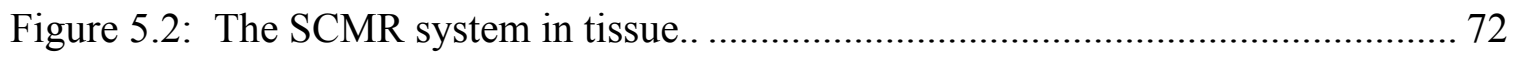

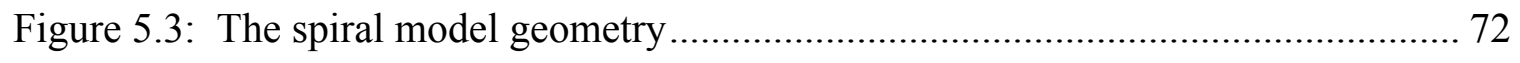

Figure 5.4: The Efficiency plots of the three different tissue types.............................. 73

Figure 5.5: The SAR distribution for SCMR system with implants........................... 74

Figure 5.6: Schematic of an SCMR system in air................................................. 76

Figure 5.7: Loop-based wireless powering systems. (a) SCMR, and (b) CSCMR. ....... 77

Figure 5.8: Performance comparison of SCMR and CSCMR ................................. 78

Figure 5.9: Multiband CSCMR: (a) Model (b) efficiency......................................... 79 


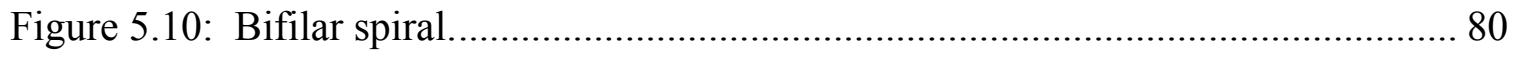

Figure 5.11: Models of three wireless powering systems. ………………………....... 81

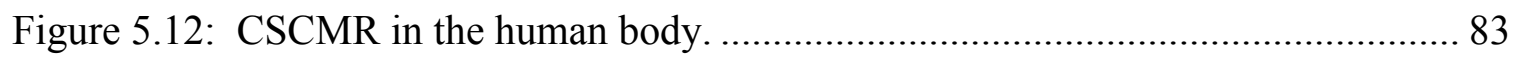

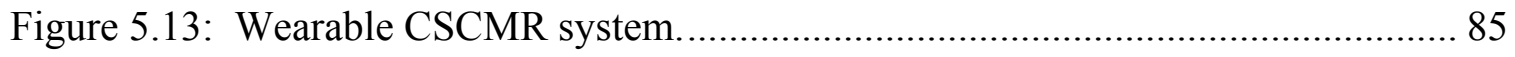

Figure 5.14: Efficiency plot human chest at $3 \mathrm{~mm}$ distance......................................... 86

Figure 5.15: Efficiency plot human head at $3 \mathrm{~mm}$ distance. ........................................ 86

Figure 5.16: Magnetic field distribution in the chest distance of $20 \mathrm{~cm}$........................ 87

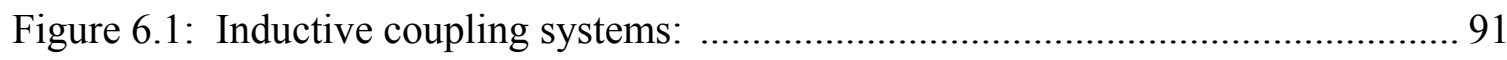

Figure 6.2: Comparison of the efficiency of the resonant and SCMR coupling........... 91

Figure 6.3: SCMR system measurement setup........................................................ 92

Figure 6.4: Q-factor of SCMR TX and RX resonators............................................... 94

Figure 6.5: Q-factor of modified TX and RX resonator................................................ 96

Figure 6.6: Q-factor of modified TX and RX resonator.............................................. 98

Figure 6.7: The three models (a) Standard RFID, standard SCMR and broadband ........ 99

Figure 6.8: The efficiency plot of the three models at $\ell_{3}=100 \mathrm{~mm}$............................ 99

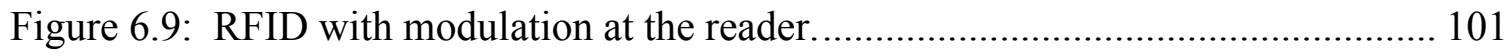

Figure 6.10: RFID with modulation at the tag........................................................... 101

Figure 6.12: Standard SCMR with modulation at the tag........................................... 102

Figure 6.14: Broadband SCMR with modulation at the tag. ....................................... 103

Figure 6.15: RFID with modulation at the reader..................................................... 104

Figure 6.16: RFID with modulation at the tag........................................................... 104

Figure 6.17: Standard SCMR with modulation at the reader....................................... 105

Figure 6.18: Standard SCMR with modulation at the tag............................................. 105 
Figure 6.19: Broadband SCMR with modulation at the reader................................ 106

Figure 6.20: Broadband SCMR with modulation at the tag. ................................... 106

Figure 7.1: SCMR system with angular elevation and angular azimuth misalignment. 109

Figure 7.2: SCMR power transfer system with lateral misalignment........................ 111

Figure 7.3: SCMR power transfer system with test for isotropy of misalignment........ 111

Figure 7.4: Schematic of an SCMR power transfer system in air. ............................ 112

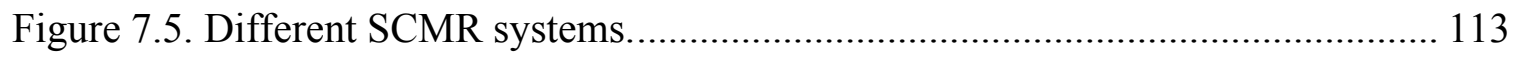

Figure 7.6: Standard SCMR with the coils different misalignment............................ 115

Figure 7.7: The modified SCMR with misalignment. ............................................ 117

Figure 7.8: SCMR with two connected orthogonal loops and two parallel................. 119

Figure 7.9: SCMR with four connected orthogonal loops ..................................... 121

Figure 7.10: The measurement setup of the misalignment insensitive SCMR ........... 122

Figure 7.11: SCMR structures with misalignment insensitivity............................... 123

Figure 7.12: SCMR with orthogonal (connected) loops, the source and load ............. 124

Figure 7.13: shows the measurement setup misalignment insensitive SCMR (3- loop) 125 


\section{LIST OF TABLES}

TABLE

PAGE

Table 2-1. Comparison of inductive coupling and SCMR ........................................ 19

Table 3-1. Helix with varying spacing, $s$............................................................. 31

Table 4-1. Extended debye's model parameters values for concrete. ........................... 47

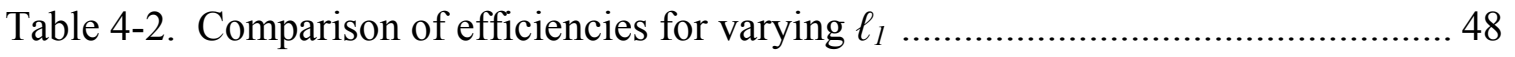

Table 4-3. Comparison of efficiencies $\ell_{3}\left(H=10 \mathrm{~cm}, D=10 \mathrm{~cm}, \ell_{3}=4 \mathrm{~cm}\right) \ldots \ldots \ldots \ldots . . . .48$

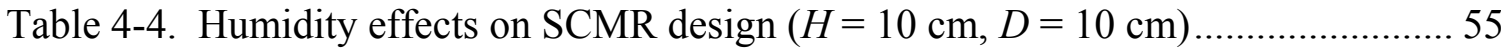

Table 4-5. Humidity effects on optimized SCMR designs $(H=10 \mathrm{~cm}, D=10 \mathrm{~cm}) \ldots . .55$

Table 4-6. Comparison of Efficiencies for varying RX depth $\left(\ell_{1}=4 \mathrm{~cm}, \ell_{3}=2 \mathrm{~cm}\right) \ldots .58$

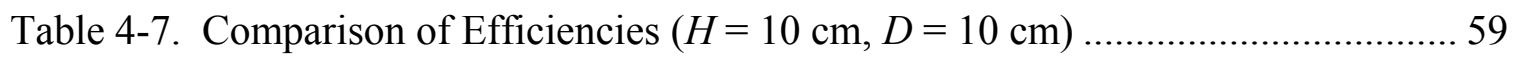

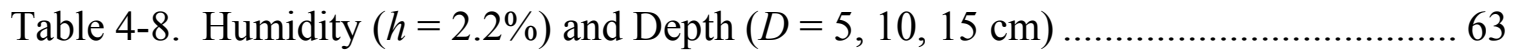

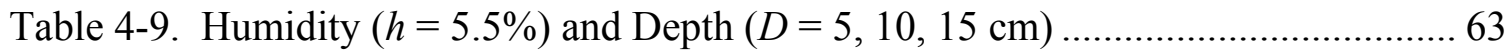

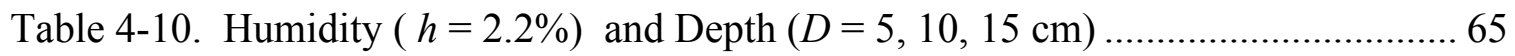

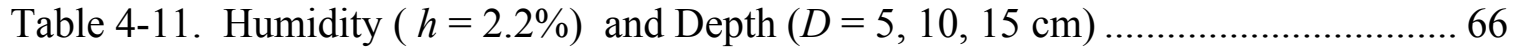

Table 5-1. Conductivities (Siemens $/ \mathrm{m}$ ) at different Tissue and Frequencies ................. 70

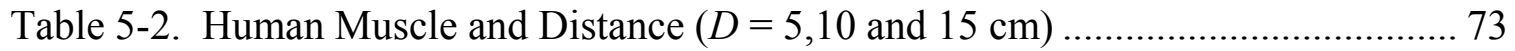

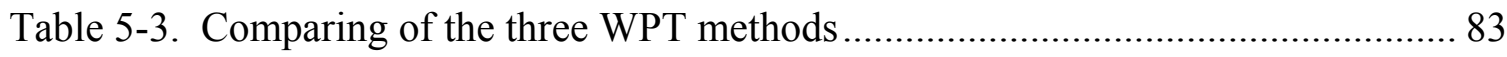

Table 5-4. Comparison of different WPT systems for IMD applications....................... 84

Table 5-5. CSCMR system at 3mm from Chest ( 1 Watt input power)......................... 86

Table 5-6. CSCMR system at $3 \mathrm{~mm}$ from Head (1 Watt input power) ......................... 86

Table 6-1. Q-factor and bandwidth of SCMR elements. .......................................... 94

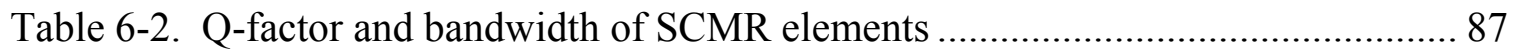




\section{CHAPTER 1}

\section{INTRODUCTION}

\subsection{Problem Statement}

A variety of non-destructive techniques (NDT) have been studied for many decades. Specifically, NDT methods, which are based on wireless solutions, have also been deployed on a wide range of applications. The three popular wireless powering techniques, which have been previously proposed and used for non-homogenous interface are: (a) inductive coupling, (b) Strongly Coupled magnetic resonance (SCMR) and (c) electromagnetic radiation. Recently, SCMR has become the subject of research work in the context of homogenous and non-homogenous interfaces. This increased interest in SCMR stems from its higher wireless powering efficiency compared to the other two methods and its ability to transfer larger amounts of power safely in the midrange. Some possible important applications of SCMR include Structural Health Monitoring (SHM) of concrete structures and Wearable and Implantable Medical Devices (WIMD). In concrete, recent scientific developments in sensors, microelectronics, and wireless communications systems coupled with several incidents of large structures (buildings, bridges, dams and airport infrastructures) collapsing without prior warning and resulting in substantial financial and great human loss of life, prompted the need for

continuous SHM. Real-time SHM systems can locate potential defects and provide crucial warnings, that will allow engineers to intervene and correct dangerous structural problems on time. SHM systems rely on sensors that can assess and report certain physical parameters, such as temperature, force, displacement, stress, humidity [1]. Unfortunately, traditional SHM systems use wired sensors that are linked by wires to a 
central station, which power the sensors and retrieve their data. The installation of wired SHM systems is expensive and time consuming. The installation of wires costs up to $25 \%$ of the total system cost and take approximately $75 \%$ of the installation time [2]. In addition, if some of the wires linked to the sensors are corroded, then these sensors become useless. Hence, novel technologies and methods need to be developed for monitoring the health of structures with minimum installation costs and with reliable performance.

SHM systems based on wireless embedded sensors have many advantages. They can be used to power sensors wirelessly, as well as retrieve the required data. SHM technologies can also be used during construction to update contractors and inspectors of vital information about the structure. In addition, wireless SHM systems allows industry and government to collect data for future construction planning, management and performance benchmarking. Hence, the development of new wireless SHM methods for construction monitoring, will provide significant benefits including reliability and cost minimization.

Wireless sensors have great potential for providing novel SHM systems. However, one of the main challenges of wireless SHM systems involves the supply of power to the embedded sensors. Traditionally, batteries can be used to power wireless sensor; however, batteries have limited lifespan. Also, the battery replacement is difficult if the sensors are embedded inside concrete structures. Hence, in order to enable a long life of the wireless sensors, novel wireless powering solutions, that can charge the rechargeable batteries of the sensors in a wireless manner are needed. Most WPT systems have been developed for transmission in the air and do not have the same performance in air-to-concrete 
transmission. Hence, the air-to-concrete transmission must be rigorously analyzed and studied in order to develop efficient and economical WPT systems.

Also, in biomedical applications, implantable medical devices (IMDs) are becoming very important as they can support artificial functions thereby saving and extending lives. IMDs can monitor, stimulate and regulate vital internal organs, and transmit to an external system detailed information about the state of health of the particular organs. IMDs have found applications in a wide range of areas, including pacemakers, physiological monitoring devices, pain relief devices, drug infusion pumps, cochlear hearing implants, functional electrical stimulators (FES), ventricular assist devices (VAD), artificial hearts, bladder-pressure monitoring devices and neurostimulators.

The power requirements of IMDs depend on the need of each application, and the typical range is from microwatts to milliwatts. Conventional operation of most IMDs relies heavily on continuous power supply from (rechargeable) batteries, which limits their applications due to their sizes and lifespan. A promising solution for this limitation is the utilization of WPT methods, which can eliminate transcutaneous wiring, and the replacement of device batteries. When developing WPT systems for IMDs, the power level is stringently restricted. This happens because too little power will result in malfunction of the IMDs, while excessive power might cause overheating of the tissue and resulting in significant tissue damage. Infact, power restrictions of IMDs require designs of low power transmitters with high power transmission efficiency as specified by the Federal Communications Commission (FCC). In addition, Wearable Medical Devices (WMD) have an increasing number of applications, WMDs are used to monitor the health 
status of elderly patients or patients receiving medical care at their house, which can reduce health care cost and improve the comfort of patients. WMDs can also be used for tracking human body movements to allow health care providers to classify and analyze a stroke patient's progress over a period of time.

Therefore, the tasks of wireless SHM and WIMD applications can be classified into the following two groups. The first group involves monitoring based on data that can be received from sensors that are placed or mounted on the surface of structures and human bodies; in such cases, the sensors in concrete can read surface: temperature, vibration, displacement, and sensors on the body can read: surface temperature, blood oxygen saturation, pulse rate and blood pressure. The second group involves monitoring based on data collected from: (a) sensors embedded in concrete that can read volumetric data, such as temperature, $\mathrm{pH}$, strain, reinforced bar corrosion, cracks, pressure, and (b) sensors embedded in the human body that monitor vital signs and functionality of organs. It should be noted that the volumetric data from embedded sensors can depict the health more accurately. Therefore, wireless embedded sensors are more suitable in many applications than surface sensors.

\section{$1.2 \quad$ Research Objectives}

The rigorous investigation of wireless power transfer systems in sensors embedded in different media (such as concrete and tissue) will lead to the development of novel and efficient non-invasive solutions in terms of cost and performance. Our research 
will concentrate on the development of novel WPT system for non-homogenous interface power transmission. Specifically, the following research tasks will be performed:

(i) Derive the optimal conditions for optimal transmission of power in homogeneous and non-homogeneous media.

(ii) Develop highly efficient WPT systems for air-to-concrete power transmission in plain and reinforced concrete.

(iii) Develop highly efficient WPT systems for air-to-human tissue power transmission.

(iv) Develop novel RFID systems that can simultaneously transfer power and data using highly efficient WPT methods.

(v) Develop novel misalignment insensitive and highly efficient WPT.

This work is expected to have significant impact to applications that strongly rely on noninvasive or non-destructive testing of concrete volumetric characteristics using wireless embedded sensors. Some of the applications include the following in concrete: (a) monitoring of temperature and humidity level (moisture) of the concrete structure, (b) collecting real-time data, such as, loads on concrete pavements, stress and displacement. In addition, our work will impact the following biomedical applications: (a) monitoring, stimulation and regulation of various vital internal organs, (b) bio-monitoring and bio sensing.

\subsection{Methodology}

This dissertation uses the Strongly Coupled Magnetic Resonance (SCMR) method to efficiently and wirelessly power sensors embedded in different media (such as, 
concrete and tissue). This work demonstrates that rigorous analysis of the air-to-concrete and air-tissue interface is critical for identifying the optimum conditions for efficient WPT.

In this research, analytical and computational methods are used to develop and design highly efficient SCMR systems in various media. In concrete, the extended Debye model is used, the model is frequency dependent. This models the properties of the concrete more accurately than fixed permittivity models. The Debye model that is used here is available for six different humidity levels. Also, the effects of reinforced bars on SCMR WPT systems are analyzed. Our results are validated by comparison of simulation with measurements.

Analytical formulations in conjunction with simulation software are also used in our analysis. Our simulations are performed using full-wave simulation software, such as, MATLAB, Mathematica, Ansoft HFSS and Ansoft Designer with Nexxim.

\subsection{Dissertation Outline}

The chapters are arranged in the following order. Chapter 2 surveys the related previous work in the field. In Chapter 3, analytical models for optimal WPT are formulated and derived. Chapter 4 develops optimal WPT systems for plain and reinforced concrete using loops, helices and spiral structures. Different rebar configurations, concrete humidity levels and placement depths are also considered. In Chapter 5, optimal WPT system for Wearable and Implantable Medical Devices (WIMD) are developed. The Specific Absorption Rate (SAR), of our systems is compared to SAR of the traditional WPT systems. In Chapter 6, novel RFID systems that simultaneously 
transfer power and data through wireless channels are developed using SCMR. In Chapter 7, misalignment insensitive and highly efficient WPT systems are developed. Chapter 8 summarizes the conclusions of this research and identifies future research directions. 


\section{CHAPTER 2}

\section{RELATED WORK}

This chapter performs the literature review of previous related work. Initially, an introduction to different wireless power transfer methods in air is presented. This is followed by a discussion of Structural Health Monitoring (SHM) in concrete including SHM wireless sensor networks with different power transfer methods. In addition, an introduction to Wearable or Implantable Medical Devices (WIMD) is presented, and it is followed by a review of different power transfer methods for biomedical applications.

\subsection{Wireless Power Transfer in Air}

\subsubsection{Inductive Coupling}

Inductive coupling has been used for several decades for WPT to two or more devices in the air. An inductive coupled power transmission system consists of two coils that are referred to as the primary and secondary coils. An overview of near field UHF RFID was presented in [3] that discusses the advantages and disadvantages of near- and far-field coupling in RFID applications. A misalignment analysis for loop antenna, which are inductively coupled, was presented in [4], which also compares the power transfer between different coil structures, such as short solenoids, with air or ferromagnetic core, planar and printed spirals. This paper also shows the maximum degree of misalignment permissible in a given application. In recent years, there has been significant interest in the development of wireless charging platforms for electric automobiles. A system that can recharge electric automobiles overnight was developed in [5]. This paper showed a 
system that delivered $2 \mathrm{~kW}$ using $700 \mathrm{~mm}$ diameter pad at a $200 \mathrm{~mm}$ distance. A contactless power system with a combination of inductive coupling technique and power electronics was developed in [6] to eliminate the risk of electric shock and short circuit. This paper also derived the optimal geometrical and electrical parameters for energy transmission using combination of finite element and theoretical analysis.

\subsubsection{Electromagnetic Radiation}

Wireless power transfer in the far-field has been performed for several decades. In 1888, Heinrich hertz transferred power via electromagnetic radiation [7]. The optimum setup for maximum power transfer between two spherical antennas was presented in [8]. In addition, the use of low-earth-orbit for the transmission of transfer power between two satellites was investigated in [9], with each satellite having a $20 \mathrm{~m}$ diameter antenna and $30 \mathrm{~m}$ rectenna with an operating range of $5-10 \mathrm{~km}$.

\subsubsection{Strongly Coupled Magnetic Resonance (SCMR)}

Strong resonant coupling employs strongly coupled magnetic elements to transmit power wirelessly and efficiently over a mid-range distance [10], where the adverse effects of the low coupling coefficient between two coils traditionally used for inductive coupling are compensated by the high-quality factor of the four-coil system to achieve higher efficiency. The strongly coupled magnetic resonance (SCMR) method is a novel non-radiative wireless mid-range power transfer method $(10-300 \mathrm{~cm})$ that has been recently developed [11]. The SCMR method achieved $40 \%$ wireless power transfer efficiency in air at a distance of $2 \mathrm{~m}$ with a single receiver element. Subsequently, this 
technique was extended to power multiple devices simultaneously in the air, and $60 \%$ efficiency was achieved at a distance of $2 \mathrm{~m}$ [12]. In addition, SCMR provides efficiencies much greater than the efficiencies of conventional resonant and non-resonant inductive coupling [13].

In [14] a three-coil multiband system was embedded in a ferromagnetic material and achieved a combined energy and data transmission with multiple receivers. Wireless power transfer system was analyzed in [15] with band-pass filter models. The paper also described a methodology for multi-receiver system using band-pass filter models and impedance matching networks to support multiple loads. The physical limitations of WPT between two electrically small devices was presented in [16], where the maximum power transfer efficiency and the optimum load impedance were described as a function of the distance between the two devices. A high power SCMR system was presented in [17] with high Q-factor spiral coils that delivered $3 \mathrm{~kW}$ power across $30 \mathrm{~cm}$ air gap with approximately $95 \%$ efficiency.

A main disadvantage of conventional SCMR systems is that they are highly sensitive to the alignment between transmitter and receiver. An optimization technique for improving the efficiency of SCMR systems under lateral misalignment was presented in [18]. Specifically, $48.4 \%$ efficiency was achieved by using an adaptive matching network. However, [18] did not provide any solution for angular misalignment. Analytical formulations for the power transfer efficiencies of loosely coupled inductive links under lateral and angular coil misalignment were presented in [19]. However, no solutions that address the effects of misalignment were discussed in [19]. The effects of changing the coil separation distance as well as the lateral and angular alignment, 
between transmitter and receiver in resonant coupling system were also examined. In addition, the ability to transfer power and data was demonstrated via resonant coupling systems in [20]. Furthermore, the effects of misalignment on the efficiency of resonant and inductively coupled RFID systems were examined using analytical formulations and FEM simulations in [21].

SCMR's radial and angular misalignment sensitivity were examined by [22], [23] respectively. Only [23], attempted to correct SCMR's angular misalignment sensitivity by using tuning circuits, which were not able to maintain high efficiency above $60^{\circ}$ of misalignment. In addition, tuning circuits add to the complexity of SCMR RX systems and they cannot compensate for large angular and radial misalignments, as they cannot recover the lost flux density between TX and RX. However, tuning circuits can be useful for compensating the effects of variable axial distance between TX and RX [24]. These papers have examined the effects of both lateral and angular misalignment of SCMR systems. However, no solutions have been proposed to address the sensitivity of SCMR systems to lateral, angular and test the performance isotropy of such systems.

\subsection{Structural Health Monitoring (SHM)}

The process of SHM necessitates the observation of a structure over a period of time for the identification of damages before a failure. It can be done repeatedly at specific times with spaced measurements to extract damage-related parameters and implement a statistical analysis of the damage-related features [25]. In [26] an adaptive learning method is presented, where stochastic models continuously evolve with the timevarying environment and Dirichlet process models are used to self-adapt to the structure 
from the data collected. In concrete structures, a variety of methods has been employed to perform the measurements and acquire damage-related data. Acoustic transducers were used in [2], to determine the corrosion of reinforced concrete structures. A wireless sensor network for SHM was designed in [27] using commercially available sensors to measure and extract the vibration characteristics of bridges with distributed processing algorithms for early damage discovery and estimation. Vehicles with ground penetrating radar (GPR) were employed in [28] to implement the nondestructive testing of bridge decks. Optical sensors were also used in [29], [30] for monitoring reinforced concrete structures. These systems can measure various quantities such as strain, temperature, moisture, and rebar corrosion. The first stage of the SHM process is sensing followed by data collection [34-36].

Extraction is the process of locating damage and fatigue area of structures after the measurement and data retrieval process. The measured data are then combined to generate their statistical distribution that will lead to the identification of possible structural damages and/or weakness. Principal component analysis was used in [34] to reduce the amount of data collected from several measurement points on a bridge column. Wavelet transforms as a time-frequency analysis method was applied in [35], [36] to find the damage location in different structures. The extraction process transforms the measured data into damage-related information, which leads to the regression analysis.

Statistical analysis is used to categorize the extracted data distributions into damaged or not damaged groups. This can be done by using either of the following methods: (a) supervised learning, and (b) unsupervised learning [37]. Supervised learning includes neural network methods [38], genetic algorithms [39], or support vector machine 
methods [40]. Unsupervised learning includes control chart analysis [15] and hypothesis testing [41], which can be used for high-level detection, such as damage, identification and classification.

Hence, the three stages of SHM enable decision making concerning structural and operational maintenance, thereby potentially saving lives, protecting investments and supporting the vitality of our economy [42], [43]. Novel SHM systems that can reduce the installation cost and complexity are needed to ensure efficient implementation of such systems. Wireless sensor networks are very suitable for such SHM systems and will be discussed in what follows [44].

\subsubsection{Wireless Sensor Networks}

Wireless sensor networks (WSNs) methods have been implemented in a wide range of applications [45], including biomedical monitoring [46], home surveillance [47], battlefield surveillance [48]. In addition, WSNs have been used and embedded in various media for monitoring purposes. Underwater sensor networks were used in oil-rig maintenance and underwater environment observations for exploration purposes in [49]. Sensors with microstrip antennas were proposed in [50] for simultaneous detection of moisture of soil and communication for agricultural research purposes, and antennas were implanted in the human body to monitor several biomedical parameters and health signals in [51]. 
Wireless monitoring systems can be classified into two groups [52]: (a) systems with surface-mounted wireless sensors, and (b) system with wireless embedded sensors [53]. Some of the advantages of WSNs are the followings:

(i) They can significantly reduce the deployment time and lower costs since wired connections are not required.

(ii) They can use wireless embedded sensors that enable monitoring process throughout the construction period, thereby enhancing the quality of the construction.

(iii) They can utilize sensors that can perform monitoring without disturbing the normal operation of the construction process.

(iv) They can be also be miniaturized in size by using compact and inexpensive sensors such as, micro-electro-mechanical systems (MEMS).

(v) They can use wireless embedded sensors that read volumetric data inside concrete thereby providing more accurate measurement results.

\subsubsection{Wireless Power Transmission for SHM}

Even though wireless sensor technologies are very suitable for SHM applications, they also have some major challenges including limited lifetime. WPT can address this issue by either charging the batteries of wireless sensors or by combining powering and communication through the same channel, thereby eliminating batteries altogether. Typical traditional wireless sensors are powered by small batteries, that can supply 
enough power for approximately 2 years with sleeping mode and energy-aware routing algorithms [54]. However, wireless sensors for SHM are required to operate up to 50 years or more, and throughout the lifespan of the structures. The process of replacing the batteries of the embedded wireless sensors is labor intensive and time consuming if not impossible. In order to ensure the long lifespan of embedded wireless sensors, novel wireless powering methods must be developed to efficiently charge sensor batteries.

Wireless powering of sensors can be done by one of the following approaches: (a) power is scavenged from contiguous energy sources, and (b) power is received from power sources via a WPT system. For example, power was scavenged from solar energy to charge a sensor less than $16 \mathrm{~mm}^{3}$ in [55], and thermal energy was converted to electrical energy by a thermo-electric generator to power micro-sensors in [56]. Thermal gradients were also converted by thermocouples to generate electrical power of approximately 2 microwatt in [57] in order to operate a small preamplifier and sensor control system. Power from human heel-strike energy was converted to electrical energy through a flexible piezoelectric foil maintained on a shoe to charge wearable microelectronic devices in [58]. Furthermore, radio frequency waves at $2.45 \mathrm{GHz}$ were harvested by a rectangular stacked patch array to charge a sensor in [59].

In addition, different WPT methods have been proposed. Inductive coupling was employed in [60] to charge and communicate with RFID tags. SCMR was used in [61] to perform power transmission in mid-range. Electromagnetic radiation was used to charge RFID tags in [62]. Traditionally, these WPT methods have been applied to air-to-air WPT. However, in many practical applications sensors are buried inside dispersive and lossy media, such as, concrete and human tissue. In order to design optimal WPT 
methods for non-homogenous power transmission, various considerations must be taken into account, such as material properties, size and the penetration of EM waves from one medium to another. In what follows, a more detailed literature review is performed for each WPT method mentioned.

\subsubsection{Inductive Coupling}

Inductive coupling has often been used in RFID, telemetry, myriad of biomedical [63] and several industrial applications [64] for decades. Inductive coupling was employed in [65] to charge SHM sensors embedded in concrete, where the reader coil was connected to a power source outside the concrete, and it was used for both powering and interrogating the embedded sensor through the magnetic coupling between the reader coil and receiving coils. A novel WSN architecture for SHM was presented in [66], and the system was based on the adoption of contactless sensors that rely on resonant coupling to sense the displacements and communicate. Passive inductive coupling systems can simultaneously perform wireless, power transmission and communication.

\subsubsection{Electromagnetic Radiation}

Electromagnetic radiation can be used to wirelessly transfer power by unitizing antennas. A $14.5 \mathrm{~dB}$ Yagi antenna transmitted $1 \mathrm{~W}$ power at $2.5 \mathrm{GHz}$ to a $19 \mathrm{~dB}$ patch antenna at a distance of $2 \mathrm{~m}$ away, to deliver power to sensors mounted on the surface of the Alamosa Canyon Bridge [67]. In addition, a stacked patch antenna with $75.8 \%$ WPT for efficiency was used to power embedded sensors experiments on the Alamosa Canyon 
Bridge in 2007 [68], and the sensor node could be charged to $3.6 \mathrm{~V}$ in $27 \mathrm{~s}$ when the power source was $1 \mathrm{~W}$ and a distance of $1.2 \mathrm{~m}$. Furthermore, a DC-to-AC converter was designed to operate at $5.7 \mathrm{GHz}$ for WPT for sensors embedded in concrete [69]. Both dry and wet concrete with different thicknesses and air-gaps were studied, and $10.37 \mathrm{~mW}$ was received at $0.6 \mathrm{~m}$ for an input power of $7 \mathrm{~W}$. A microstrip antenna operating at 2.45 GHz was embedded in concrete for SHM in [70], and the performance was dependent on the concrete dielectric constant and loss tangent. Also, in [71] dipole and PIFA antennas operating at $915 \mathrm{MHz}$ were embedded inside a concrete platform.

Even though several electromagnetic radiation WPT systems have been proposed, they all exhibit very low efficiencies thereby wasting a significant amount of power and limiting the maximum power that can be transmitted while satisfying the pertinent RF safety standards.

\subsection{Wearable and Implantable Medical Devices (WIMD)}

Wearable and Implantable Medical Devices (WIMD) are gaining prominence and are expected to play a significant role in saving and extending human lives, due to their ability to monitor, stimulate and regulate vital internal organs, and also communicate with an external host about the state of health of the these internal organs. They have been applied in a wide range of areas, including pacemakers, physiological monitoring devices, pain relief devices, drug infusion pumps, cochlear hearing implants, functional electrical stimulators (FES), left ventricular assist devices (LVAD) [72], artificial hearts, bladder-pressure monitoring devices [73], and Neuro-stimulators [74]. 
The power requirement of WIMDs depends on the specific application, and the typical range is from microwatts [75] to milliwatts [76]. Conventional operation of most WIMDs relies heavily on continuous power supply from batteries, which, however, limit the range applications due to the size and/or lifetime. A promising solution for this limitation is the use of WPT systems that can avoid transcutaneous wiring and the replacement of batteries. When developing WPT systems for WIMDs, the power levels are strictly regulated. Too little power can lead to malfunction of the device, while extra power can cause excessive heat or damage in the surrounding tissues [77]. This power restriction requires the design of low power transmitters that can wirelessly transfer power with high efficiency [78]. Three WPT methods are reviewed below in the context of air-to-tissue transmission.

\subsubsection{Inductive Coupling}

Inductive coupling was first used to charge an artificial heart in 1961 and since then has been commonly used for wireless charging of IMDs. An inductive coupled power transmission system consists of two coils that are referred to as primary and secondary coils. The efficiency of the system is closely related to the quality factor of coils, and depends on the size, geometry, physical spacing, relative location and surrounding material [79]. A dual band telemetry for a retinal prosthetic device is proposed in [80], [81] to ensure both the power transmission efficiency and higher data rate. The optimization of WPT through inductive links along with their sensitivity is studied in [82]. An inductive WPT system for endoscope micro-robot was proposed in [83]. Optimal printed spiral coils were presented in [84], [85] to provide maximum WPT 
efficiency. Numerical methods that can be used for the design of coupled coils for single and bi-directional communication links via electromagnetic energy transfer at low frequencies in homogenous and non-homogenous media were presented in [86].

\subsubsection{Strongly Coupled Magnetic Resonance (SCMR)}

The SCMR method was extended to in-vitro and in-vivo experiments in [87]. In addition, the SCMR method was optimized for biomedical implant applications in [60]. A comparison of the performance of two-coil inductive coupling methods with SCMR is performed in Table 2-1.

Table 2-1. Comparison of inductive coupling and SCMR

\begin{tabular}{|c|c|c|c|c|}
\hline Reference & Dimension $\left(\mathrm{r}_{\mathrm{p}}, \mathrm{r}_{\mathrm{s}}\right)(\mathrm{mm})$ & Freq. $(\mathrm{MHz})$ & Efficiency & Distance $\left(\mathrm{d}(\mathrm{mm}), \mathrm{d} / \mathrm{r}_{m}\right)$ \\
\hline$[79]$ & $(15,15)$ & 4.5 & $54 \%$ & $(10,0.67)$ \\
\hline$[80]$ & $(8.5,6)$ & 1.0 & $30 \%$ & $(7,0.98)$ \\
\hline$[81]$ & $(30,10)$ & 0.7 & $36 \%$ & $(30,1.73)$ \\
\hline$[82]$ & $(26,5)$ & 6.78 & $22 \%$ & $(15,1.73)$ \\
\hline$[83]$ & $(12,12)$ & 2.0 & $40 \%$ & $(12,1)$ \\
\hline$[84]$ & $(35,10)$ & 5.0 & $30 \%$ & $(20,1.07)$ \\
\hline$[78]$ & $(32,10)$ & 0.7 & $82 \%$ & $(20,1.07)$ \\
\hline
\end{tabular}

$r_{p}$ and $r_{s}$ are the radius of primary and secondary coils, while $r_{m}$ is the geometric mean of $r_{p}$ and $r_{s}$.

In addition, an SCMR system for wireless power transfer to small biomedical implantable was presented in [88], it also examined axial and angular misalignments at $15 \mathrm{~mm}$ axial distance and misalignments angle of $50^{\circ}$. A miniaturized SCMR system operating at $6.78 \mathrm{MHz}$ integrated with a Medical Implant Communication Service (MICS) antenna and system operating in the $402-405 \mathrm{MHz}$ band was presented in [89]. The SCMR system was used for WPT and the MICS system was used for the data communication. This system achieved a WPT efficiency of $35 \%$ at $20 \mathrm{~mm}$ distance . 


\subsubsection{Electromagnetic (EM) Radiation}

WPT based on EM radiation has also been used for powering WIMDs. A full wave analysis was performed in [90] in order to identify the optimal frequency for power transmission in biological media at GHz-range and at a depth of $2 \mathrm{~cm}$. A PIFA antenna operating at $2.45 \mathrm{GHz}$ was proposed in [91] for microwave heating, radiometry and implant communication applications, with the dimension of $5 \mathrm{~mm} \times 4 \mathrm{~mm}$.

\subsection{Summary}

In this chapter, the literature review of SHM methods has been presented. In addition, various WPT and power harvesting methods were reviewed for charging embedded sensors wirelessly. Furthermore, inductive coupling, electromagnetic radiation and SCMR methods are reviewed in the context of for WIMDs. Inductive coupling is widely used and several designs have been proposed based on it in the past. The electromagnetic radiation method has small antenna size, and can support longer distance of power transmission, but its efficiency is very small (low). SCMR has the largest power transmission efficiency in the air, and should be explored for applications in SHM and WIMD. 


\section{CHAPTER 3}

\section{OPTIMIZATION FOR STRONGLY COUPLED MAGNETIC RESONANCE}

In this chapter, the conditions for the optimal design of the SCMR elements are

derived. Specifically, the conditions for optimal Q-factor and maximum efficiency are derived using analytical formulations for loops, helices and spirals.

\subsection{Wireless Powering via SCMR}

Numerous wireless power transfer methods have been proposed and studied in the past for various applications. Specifically, wireless power transfer has been achieved using near-field coupling in several applications such as, RFID tags, telemetry and implanted medical devices [92], [3]. In addition, certain inductive coupling techniques have been reported to exhibit high power transfer efficiencies greater than $90 \%$ at very short distances $(1-3 \mathrm{~cm})$ [93]. However, the efficiency of such techniques drops drastically for longer distance since it decays as $1 / \mathrm{r}^{6}$ [94], [95].

The SCMR method is a novel non-radiative mid-range wireless power transfer method $(10-300 \mathrm{~cm})$ that has been recently developed [10]. In fact, the SCMR method has achieved $40 \%$ wireless power transfer efficiency in the air at a distance of $2 \mathrm{~m}$ for a single receiver [11] where it delivered $60 \mathrm{~W}$. Also, SCMR technique was used to simultaneously power multiple receivers in air, and achieving $60 \%$ efficiency at a distance of $2 \mathrm{~m}$ [12]. Recent papers have shown that SCMR provides wireless power transfer efficiencies that are significantly larger than efficiencies of conventional inductive coupling methods [13], [96]. In order for SCMR to achieve high efficiency, it requires that the transmitting and receiving elements (typically loops, coils or spirals) are 
designed so that they resonate at the desired operating frequency that must coincide with the frequency where the elements exhibit maximum Q-factor. In this chapter, we analytically derive equations that must be satisfied by the geometrical parameters of loops, helices or spirals in order to guarantee maximum efficiency of the SCMR systems.

Strongly coupled systems are able to transfer energy efficiently, because resonant objects exchange energy efficiently versus non-resonant objects that only interact weakly. A standard SCMR system consists of four elements (typically four loops or two loops and two coils) as shown in Figure 3.1 [10].

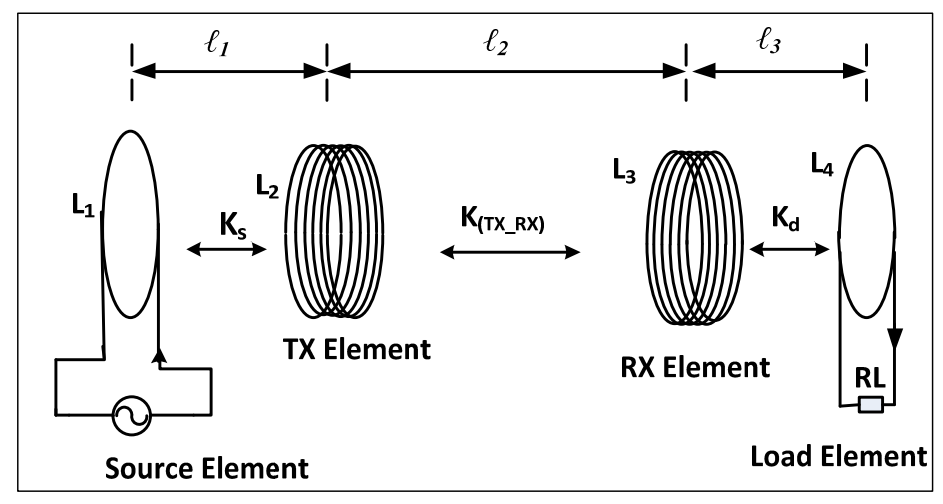

Figure 3.1: Schematic of an SCMR power transfer system in air. $K_{\mathrm{S}}, K_{\mathrm{TX} \_\mathrm{RX}}$ and $K_{\mathrm{d}}$ are the respective coupling coefficients.

The source element is connected to the power source, and it is inductively coupled to the TX element. The TX element exhibits a resonant frequency that coincides with the frequency, where its Q-factor is naturally maximum. Similarly, the RX element exhibits a resonant frequency that coincides with the frequency where its Q-factor is naturally maximum. Furthermore, the load element is terminated with a load. For our analysis, we assume that the entire system operates in air. Also, SCMR requires that the TX and RX elements are resonant at the same frequency in order to achieve the efficient wireless power transfer. The optimization analysis of the different elements is discussed next. 


\subsection{Optimal Loop and Helical Structures for SCMR}

In this section, we will derive the guidelines for designing SCMR systems that use loops and helices as TX and RX elements. The TX and RX elements can be equivalently represented by a series RLC circuit. Helices are often preferred in SCMR system TX and RX elements because they exhibit both distributed inductance and capacitance and therefore, they can be designed to self-tune to a desired resonant frequency, $f_{r}$, without the need of external capacitors. In addition, external capacitors have losses, which in practice can reduce the Q-factor of the TX and RX elements and in turn decrease the efficiency of SCMR systems. Based on the equivalent RLC circuit of an SCMR system, its resonant frequency, $f_{r}$, can be calculated by the following equation:

$$
f_{r}=\frac{1}{2 \pi \sqrt{L C}}
$$

The resonant frequency, $f_{r}$, is also the operational frequency of the SCMR wireless powering system. The Q-factor of a resonant RLC circuit is given by:

$$
Q=\frac{\omega_{r} L}{R}=\frac{2 \pi f_{r} L}{R}
$$

Therefore, the Q-factor of a resonant helix (i.e., self-resonant) can be written as:

$$
Q=\frac{2 \pi f_{r} L}{R_{o h m}+R_{r a d}}
$$

where $L, R_{\text {rad }}$, and $R_{o h m}$ are the self-inductance, radiation resistance and ohmic resistance of the helix, which are given by [102-104] [94].

$$
L=\mu_{o} r N^{2}\left[\ln \left(\frac{8 r}{r_{c}}\right)-2\right]
$$




$$
\begin{gathered}
R_{\text {rad }}=(\pi / 6) \eta_{o} N^{2}\left(2 \pi f_{r} r / c\right)^{4} \\
R_{o h m}=\left(\sqrt{\mu_{o} \rho \pi f_{r}}\right) N r / r_{c}
\end{gathered}
$$

where $\mu$ is the permeability of free space, $\rho$ is the helix's material resistivity, $r$ is the radius of the helix, $r_{c}$ is the cross sectional wire radius, $N$ is the number of turns, $f$ is the frequency, $\eta_{o}$ is the impedance of free space and $c$ is the speed of light. It should also be noted that (3.3)-(3.6) are valid only when $r<\lambda / 6 \pi$ [93]. It is important to note (3.1) -(3.6) can be used to describe a loop $(N=1)$, which will have a negligible selfcapacitance. Therefore, in order to resonate a loop element, it must be connected to an external capacitor in order to form a resonant series RLC circuit.

SCMR requires that both $\mathrm{RX}$ and $\mathrm{TX}$ helices also exhibit maximum Q-factor at their resonant frequency $f_{r}$, in order to achieve maximum wireless power efficiency. This can also be seen from the following equation that describes the efficiency of an SCMR system at its operational frequency, $f r$, as follows [100]:

$$
\eta\left(f_{r}\right)=\frac{k_{\left(T X_{-} R X\right)}^{2}\left(f_{r}\right) Q_{T X}\left(f_{r}\right) Q_{R X}\left(f_{r}\right)}{1+k_{\left(T X_{-} R X\right)}^{2}\left(f_{r}\right) Q_{T X}\left(f_{r}\right) Q_{R X}\left(f_{r}\right)}
$$

where $K_{T X \_} R X$ is the mutual coupling between the RX and TX helices, whereas $Q_{T X}$ and $Q_{R X}$ are the Q-factors of the RX and TX helices respectively. If the TX and RX helices are identical their Q-factors are equal, i.e., $Q_{T X}=Q_{R X}=Q$; therefore equation (3.7) can be written as:

$$
\eta\left(f_{r}\right)=\frac{k_{\left(T X_{-} R X\right)}^{2}(f) Q_{T X}^{2}\left(f_{r}\right)}{1+k_{\left(T X_{-} R X\right)}^{2}\left(f_{r}\right) Q_{T X}^{2}\left(f_{r}\right)}
$$


Equation (3.8) shows that in order to maximize the efficiency of an SMCR system, the operation frequency $f_{r}$ must be equal to the frequency $f_{\max }$, where the Q-factor is maximum. In what follows, the maximum Q-factor of a resonant helix is derived. The Q-factor of a resonant helix can be expressed in terms of its geometric parameters using $(3.3)-(3.6)$ as:

$$
Q\left(f_{r}, r, r_{c}, N\right)=\frac{2 \pi f_{r} \mu_{0} r N^{2}\left[\ln \left(\frac{8 r}{r_{c}}\right)-2\right]}{\left(\frac{\mu_{0} \rho \pi f_{r} r^{2} N}{r_{c}^{2}}\right)^{\frac{1}{2}}+20 \pi^{2} N^{2}\left(\frac{2 \pi f_{r} r}{c}\right)^{4}}
$$

The maximum Q-factor, $Q_{\max }$, and the frequency, $f_{\max }$, where $Q_{\max }$ occurs, can be derived from (3.9) using calculus as:

$$
\begin{gathered}
f_{\max }\left(r, r_{c}, N\right)=\frac{c^{8 / 7} \mu^{1 / 7} \rho^{1 / 7}}{4 \cdot 15^{2 / 7} N^{2 / 7} r_{c}^{2 / 7} \pi^{11 / 7} r^{6 / 7}} \\
Q_{\max }\left(r, r_{c}, N\right)=\frac{2 \pi f_{\max } \mu_{0} r N^{2}\left[\ln \left(\frac{8 r}{r_{c}}\right)-2\right]}{\left(\frac{\mu_{0} \rho \pi r^{2} f_{\max } N}{r_{c}^{2}}\right)^{\frac{1}{2}}+20 \pi^{2} N^{2}\left(\frac{2 \pi f_{\max } r}{c}\right)^{4}}
\end{gathered}
$$

Based on the above discussion, an SCMR system requires that the following equations should be satisfied for maximum power transfer:

$$
f_{r}=f_{\max }
$$

which can be written based on (3.10) as:

$$
f_{r}\left(r, r_{c}, N\right)=\frac{c^{8 / 7} \mu^{1 / 7} \rho^{1 / 7}}{4 \cdot 15^{2 / 7} N^{2 / 7} r_{c}^{2 / 7} \pi^{11 / 7} r^{6 / 7}}
$$


Therefore, (3.13) shows that the geometrical parameters of a helix can be appropriately chosen so that the helix has maximum Q-factor at a chosen frequency, $f_{r}$. For example, if the parameters $f_{r}, r_{c}, N$ and $\rho$ are specified by a designer, (3.13) can be solved for the radius of the maximum Q-factor, $r_{\max }$, as follows:

$$
r_{\max }=\left[\frac{c^{8 / 7} \mu^{1 / 7} \rho^{1 / 7}}{4 \cdot 15^{2 / 7} r_{c}^{2 / 7} N^{2 / 7} \pi^{11 / 7} f_{r}}\right]^{7 / 6}
$$

Similar equations to (3.10), (3.11) and (3.13) have been presented in [101] in order to obtain the local maximum for the Q-factor. Next, the case of a loop (with $N=1$ ) is analyzed using (3.10), (3.11) and (3.14) to study the behavior of the maximum Qfactor, $Q_{\max }$, versus the electrical length of the loop $\left(C_{\text {dev }} / \lambda_{Q_{\max }}\right)$ at $f_{\max }$, which can be written as:

$$
\frac{C_{d e v}}{\lambda_{\max }}=\frac{2 \pi r_{\max }}{\lambda_{\max }}=\frac{2 \pi r_{\max } f_{\max }}{c}
$$

where $C_{d e v}$ is the circumference of the loop (device), $\lambda_{\max }$ is the wavelength corresponding to $f_{\max }$ given by (3.10). Here, optimum SCMR loops are designed in the frequency range $100 \mathrm{KHz} \leq f \leq 100 \mathrm{MHz}$ for four values of the cross-sectional radius, $r_{c}$ $=0.01,0.1,1.0$ and $5 \mathrm{~mm}$. The material of the loops is assumed copper and for each pair of $f_{\max }$ and $r_{c}$, the optimum $r$ is calculated by (3.14). Then $Q_{\max }$ from (3.11) is plotted in Figure 3.2 versus the electrical length of the loop $\left(C_{d e v} / \lambda_{Q_{\max }}\right)$, which is calculated by (3.15). Specifically, Figure 3.2 illustrates that for each pair of $f_{\max }$ and $r_{c}$ there is an $r_{\max }$ that provides the global maximum for the Q-factor, $Q_{G \max }$. The 3D plot of the local, global optimal resonant frequency and set of optimal Q-factor are shown in Figure 3.3. 


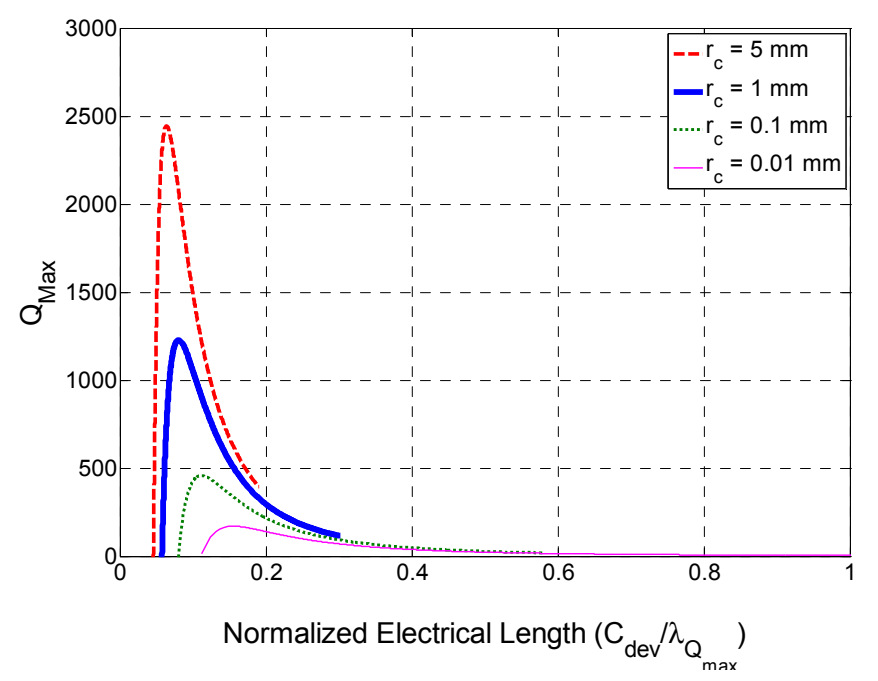

Figure 3.2: $Q_{\max }$ vs. electrical length of the loop.

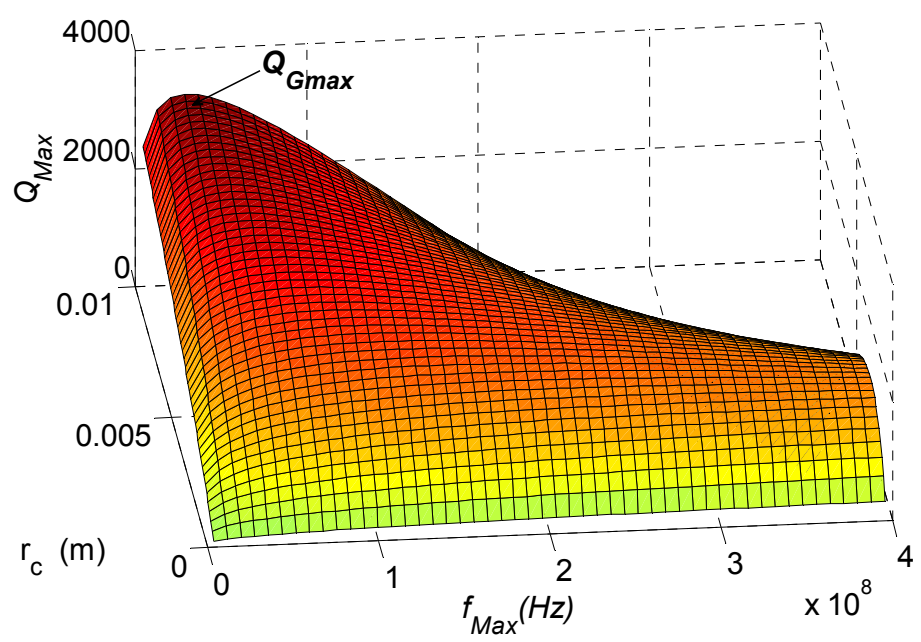

Figure 3.3: Local maximum Q-factor $\left(Q_{\max }\right)$ vs. cross-sectional radius $\left(r_{c}\right)$ and optimal frequency $\left(f_{\max }\right)$.

In what follows the global maximum Q-factor of the helix, $Q_{G \max }$, is formulated.

First, the local maximum Q-factor, $Q_{L \max }$, is derived by substituting (3.10) into (3.11):

$$
\left.Q_{L \max }=\frac{2 \cdot 3^{6 / 7} r_{c}^{6 / 7} c^{8 / 7} \mu^{8 / 7} N^{6 / 7} \rho^{1 / 7}\left[\ln \left(\frac{8 r}{r_{c}}\right)-2\right]}{5^{1 / 7} \pi^{2 / 7} r^{3 / 7}\left[c^{4 / 7} \mu^{4 / 7} \rho^{4 / 7}+6 r_{c}^{1 / 7} N^{1 / 7} r^{3 / 7} \sqrt{\frac{c^{8 / 7} \mu^{8 / 7} \rho^{8 / 7}}{r_{c}^{2 / 7} N^{2 / 7} r^{6 / 7}}}\right.}\right]
$$


Using again calculus, we can find out that the global maximum of Q-factor occurs when:

$$
\frac{r_{(G \max )}}{r_{c}}=\frac{e^{13 / 3}}{8} \approx 9.52
$$

This is an important finding, which shows that the ratio between the helix radius, $r$, and the cross-sectional radius, $r_{c}$, must be approximately 9.52 in order to achieve the maximum Q-factor. This ratio is also independent of frequency and material. Also, by substituting (3.17) into (3.16) we can write the global maximum for the Q-factor as:

$$
Q_{G \max }=\frac{28 \cdot 2^{2 / 7} r_{c}^{3 / 7} c^{8 / 7} \mu^{8 / 7} N^{6 / 7} \rho^{1 / 7}}{15^{1 / 7} e^{13 / 7} \pi^{2 / 7}\left[c^{4 / 7} \mu^{4 / 7} \rho^{4 / 7}+6 r_{c}^{4 / 7} N^{1 / 7} \sqrt{\frac{c^{8 / 7} \mu^{8 / 7} \rho^{8 / 7}}{r_{c}^{8 / 7} N^{2 / 7}}}\right]}
$$

Therefore, if a helix is designed to operate at the global maximum Q-factor it will yield the maximum possible wireless efficiency for the corresponding SCMR system. In order to verify the global maximum design of (3.17), we assume that an arbitrary ratio of $r / r_{c}=t$, and solve (3.13) to obtain the $r$ and $r_{c}$ given the number of turns, $N$, and the desired frequency of operation, $f_{o}$ :

$$
\begin{gathered}
r_{c_{\max }}=\frac{c \mu^{1 / 8} \rho^{1 / 8}}{2 \cdot 2^{3 / 4} \cdot 15^{1 / 4} N^{1 / 4} f_{o}^{7 / 8} \pi^{11 / 8} t^{3 / 4}} \\
r_{\max }=\frac{c \mu^{1 / 8} \rho^{1 / 8} t^{1 / 4}}{2 \cdot 2^{3 / 4} \cdot 15^{1 / 4} N^{1 / 4} f_{o}^{7 / 8} \pi^{11 / 8}}
\end{gathered}
$$

Based on (3.19) and (3.20), SCMR systems were designed and simulated in Ansoft HFSS for different ratios $r / r_{c}(2 \leq \mathrm{t} \leq 50)$ and assuming the number of turns, $N=$ 1, distances, $\ell_{1}=\ell_{3}=2 \mathrm{~cm}, \ell_{2}=7 \mathrm{~cm}$ (see Figure 3.1), and operational frequency, $f_{o}=$ $197 \mathrm{MHz}$. The efficiency of these designs is compared in Figure 3.4. The results clearly 
illustrate that the maximum efficiency is achieved for a ratio of $t=9.52$ that matches our derived global maximum condition of (3.17).

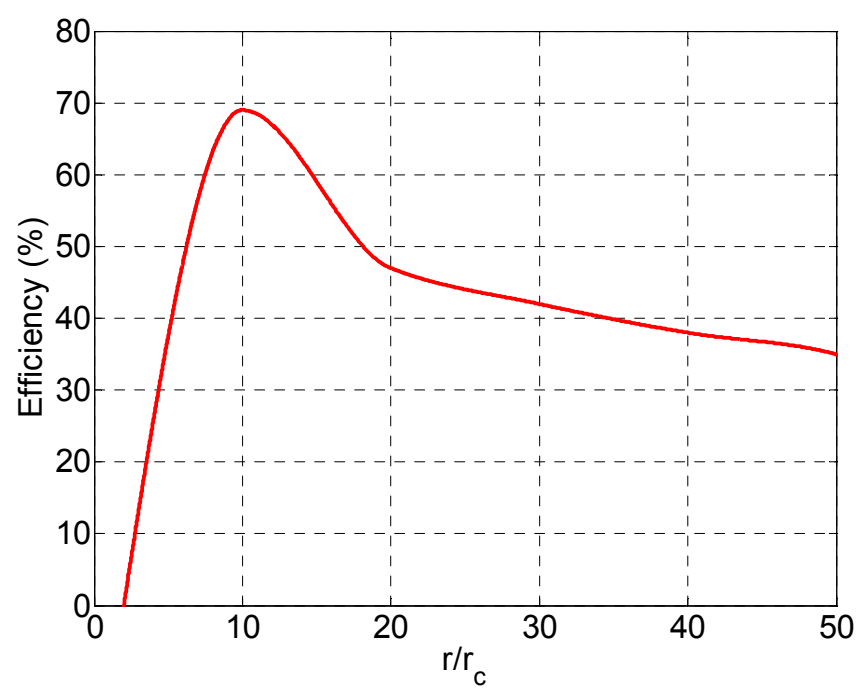

Figure 3.4: The efficiency of an SCMR system for different $r / r_{c}$ ratios.

\subsubsection{Optimal Spacing of SCMR Helices}

In this section, we will examine the guidelines for designing helical TX and RX elements of SCMR wireless powering systems. An SCMR system based on helices will not be optimal unless the spacing, $s$, is picked so that the helices exhibit the appropriate capacitance in order to resonate at the desired operating frequency of the system. In [102], it was also shown that the spacing, $s$ of an SCMR helix is an important parameter that should be carefully picked to ensure optimal wireless power transfer efficiency. The capacitance formula for closely wound helix was presented in [103] as follows:

$$
C_{t}=\frac{2 \pi^{2} r \varepsilon_{0}}{\ln \left[s / 2 r_{c}+\sqrt{\left(s / 2 r_{c}\right)^{2}-1}\right]}
$$


where $r$ is the radius of the helix, $r_{c}$ is the cross sectional wire radius, $\varepsilon_{0}$ is the permittivity of free space, $s$ is the spacing between adjacent turns of the helix, $C_{t}$ is the total distributed capacitance of the helix, and $t$ is the thickness of the insulation coating.

The capacitance formula of (3.21) is valid when $s / 2 r_{c} \leq 2$ and $t<<s-2 r_{c}$. In order to resonate the helix at a desired frequency $f$, the spacing between two adjacent turns, $s$, can be adjusted to provide the required capacitance calculated from (3.1) as:

$$
C_{t}=\frac{1}{4 \pi^{2} f^{2} L_{h e l i x}}
$$

Then equation (3.21) can be solved for the optimal spacing, $\mathrm{s}_{\max }$, as follows:

$$
S_{\max }=\frac{\left[e^{\left(\frac{4 \pi^{4} r^{2} \varepsilon_{0}^{2}}{C_{t}^{2}}\right)}+1\right] r_{c}}{e^{\left(\frac{2 \pi^{2} r \varepsilon_{0}}{C_{t}}\right)}}
$$

Therefore, the spacing, s, can be adjusted using (3.23) independently from the other geometrical parameters to achieve the necessary capacitance and without affecting the frequency where a helix exhibits maximum Q-factor since (3.13) shows that the $f_{\max }$ does not depend on $s$.

In the case of a loop $(N=1)$, only self- inductance, $L$, exists and negligible selfcapacitance. Therefore, in order to resonate a loop element, it must be connected to a capacitor in order to form a resonant series RLC circuit. The capacitance required to resonate a loop at a frequency, $f$, is calculated from (3.22).

Next, we examine the performance of SCMR system using helices with number of turns, $N=6$, coil radius, $r=10 \mathrm{~cm}$, cross-sectional radius, $r_{c}=2.2 \mathrm{~mm}$, distance 
between helices, $\ell_{2}=40 \mathrm{~cm}$ and the spacing, $\mathrm{s}$ is varied. The table $3-1$ clearly shows that there is an optimal spacing for efficient power transfer. Figure 3.5 also illustrates the effect of spacing on SCMR's efficiency.

Table 3-1. Helix with varying spacing, $s$

\begin{tabular}{|l|l|l|l|l|l|}
\hline Helix spacing & $6 \mathrm{~mm}$ & $10 \mathrm{~mm}$ & $20 \mathrm{~mm}$ & $30 \mathrm{~mm}$ & $50 \mathrm{~mm}$ \\
\hline Efficiency (HFSS) & $10.7 \%$ & $59 \%$ & $80.1 \%$ & $61.8 \%$ & $34.7 \%$ \\
\hline $\begin{array}{l}\text { Frequency of } \\
\text { Maximum } \\
\text { efficiency (HFSS) }\end{array}$ & $15.6 \mathrm{MHz}$ & $22.5 \mathrm{MHz}$ & $28.1 \mathrm{MHz}$ & $31.7 \mathrm{MHz}$ & $37.1 \mathrm{MHz}$ \\
\hline $\begin{array}{l}Q_{\max } \text { and } f_{\max } \\
\text { (Analytical) (MHz) }\end{array}$ & $\begin{array}{l}Q_{\max }=4913, \\
f_{\max }=26.94\end{array}$ & $\begin{array}{l}Q_{\max }=2948, \\
f_{\max }=26.94\end{array}$ & $\begin{array}{l}Q_{\max }=1474, \\
f_{\max }=26.94\end{array}$ & $\begin{array}{l}Q_{\max }=983, \\
f_{\max }=26.94\end{array}$ & $\begin{array}{l}Q_{\max }=583, \\
f_{\max }=26.94\end{array}$ \\
\hline
\end{tabular}

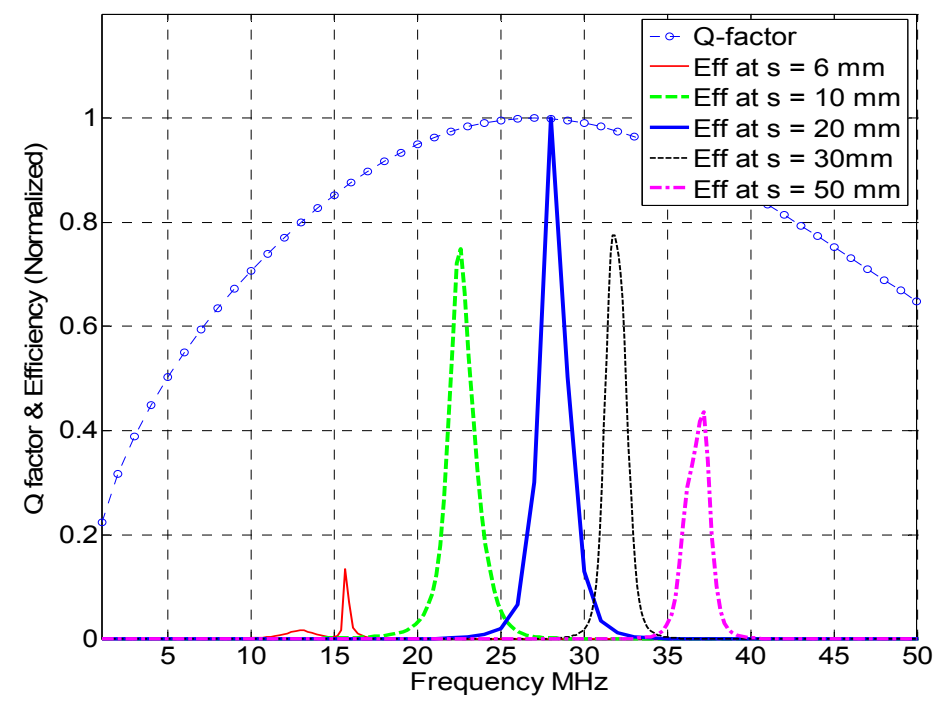

Figure 3.5: The Q-factor and efficiency of helical SCMR system with different spacing $\left[r=10 \mathrm{~cm}, r_{c}=2.2 \mathrm{~mm}, \ell_{2}=40 \mathrm{~cm}\right]$.

\subsection{Optimal Spirals for SMCR}

A standard SCMR system based on spirals is shown in Figure 3.6. The setup of the system in Figure 3.6 is similar to the previous arrangement in Figure 3.1, and all corresponding parameter names are the same as previously defined. 


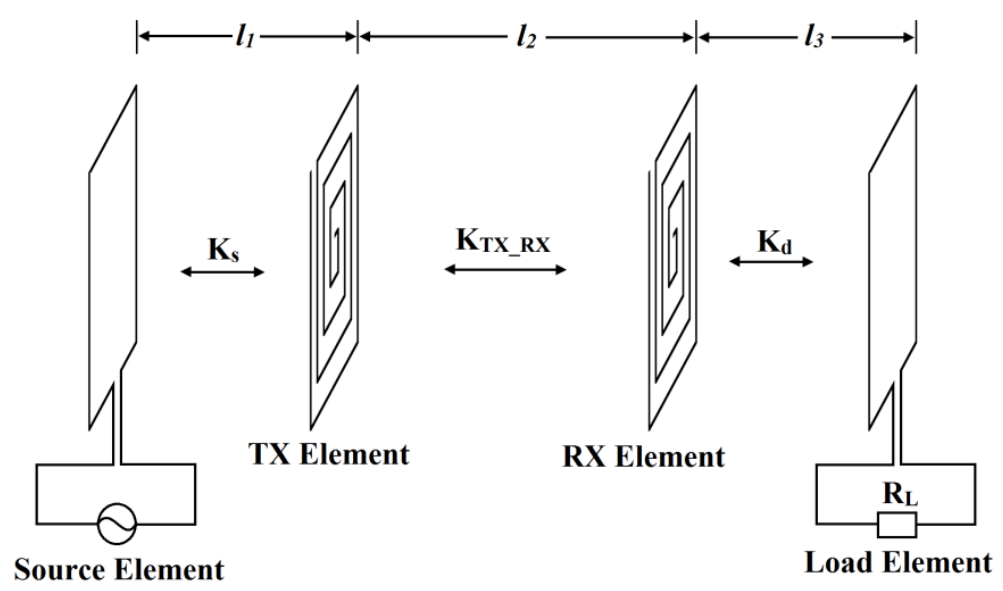

Figure 3.6: The schematic of an SCMR system with spirals in the air, where $K_{\mathrm{S}}, K_{\mathrm{TX} \_\mathrm{RX}}$ and $K_{\mathrm{d}}$ are the respective coupling coefficients.

The TX and RX resonators shown in Figure 3.6 can be equivalently represented by an RLC circuit shown in Figure 3.7. Helices and spirals are often preferred as SCMR TX and RX resonators because they exhibit both distributed inductance and capacitance thereby requiring no external capacitors to tune to the self-resonant frequency. Also, external capacitors have losses, which in practice can reduce the Q-factor of the TX and RX elements and in turn decrease the efficiency of SCMR systems.

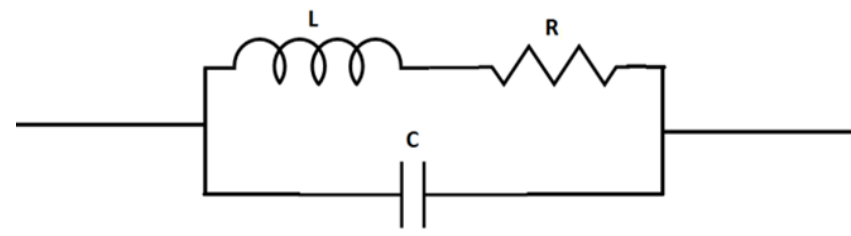

Figure 3.7: The RLC representation of a spiral.

Figure 3.8 shows a square spiral with a rectangular cross-section. The basic dimensional parameters of such spiral are $N, W, S, T$ and $d_{\text {out }}$ which are the number of turns, cross-sectional width, spacing between turns, thickness of the trace material, and the outermost side length of the spiral, respectively. 


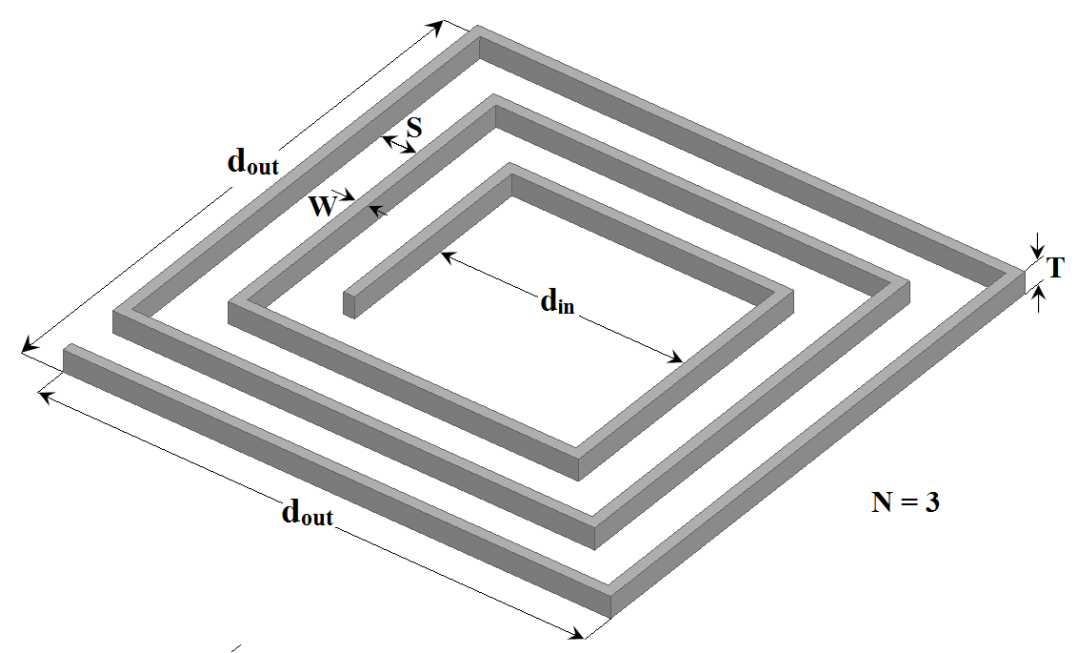

Figure 3.8: The spiral model geometry.

The inner diameter, $d_{\text {in }}$, is derived from the other geometrical parameters as:

$$
d_{\text {in }}=d_{\text {out }}-2[N K-S]
$$

where $K=W+S$, is the distance between the centers of two adjacent turns. The total length, $\ell_{\text {tot }}$, of the spiral can be calculated as:

$$
\ell_{\text {tot }}=4 N\left[d_{\text {out }}-K(N-1)\right]
$$

The resonant frequency, $f_{r}$ is also the operational frequency of the SCMR wireless powering system. The $f_{\mathrm{r}}$ can be calculated from (3.1) and the Q-factor from (3.3). The inductance, $L$, of a spiral can be written as [104]:

$$
L=\left[\frac{\mu_{\mathrm{o}} N^{2}\left(\frac{d_{\text {in }}+d_{\text {out }}}{2}\right) c_{1}}{2}\right]\left[\ln \left(\frac{c_{2}}{\alpha}\right)+c_{3} \alpha+c_{4} \alpha^{2}\right]
$$


where $c_{1}=1.27, c_{2}=2.07, c_{3}=0.18$ and $c_{4}=0.13$, are the constants derived based on the geometrical layout of the square spiral; and $\alpha$ is the fill ratio defined by $\alpha=\left(d_{\text {in }}-d_{\text {out }}\right) /\left(d_{\text {in }}+d_{\text {out }}\right)$. The ohmic and radiation resistances can be written as [94] :

$$
\begin{aligned}
R_{\mathrm{ohm}} & =\frac{\ell_{\mathrm{tot}}}{4 \sqrt{W T}} \sqrt{\pi \mu_{\mathrm{o}} \rho f}\left(1+\frac{R_{\mathrm{p}}}{R_{\mathrm{o}}}\right) \\
R_{\mathrm{rad}} & =31200\left(\frac{f}{c}\right)^{4}\left(\sum_{i=1}^{N} d_{i}^{2}\right)^{2}
\end{aligned}
$$

where, $d_{i}$ is the side length of the $i$ th turn of the spiral, $\rho$ is the spiral's conductor resistivity, $c$ is the speed of light, and $\sqrt{\pi \mu_{\mathrm{o}} \rho f}$ represents the conductor's sheet resistance [94]. The factor $R_{\mathrm{p}} / R_{\mathrm{o}}$ in (3.27) represents the proximity effect factor that accounts for the additional resistance due to the closeness of the conductors. The proximity factor depends on $W, S$ and $N$ and adds additional resistance that is undesirable as it reduces the Q-factor. Hence, the spiral dimensions have to be chosen carefully to maximize the Q-factor. Specifically, the proximity factor can be significantly reduced by increasing the spacing between turns, $S$, and decreasing the width, $W,(S>5 W)$ [105]. In order to derive analytical expressions for $Q_{\max }$ and $f_{\max }$, the analytical and simulation setups are chosen such that the proximity effect is negligible reducing (3.28) to:

$$
R_{\mathrm{ohm}}=\frac{\ell_{\mathrm{tot}}}{4 \sqrt{W T}} \sqrt{\pi \mu_{\mathrm{o}} \rho f}
$$

It should also be noted that (3.27) - (3.29) are effective in SCMR analysis only when $\ell_{\text {tot }}<\lambda / 3$ [94]. The Q-factor of a resonant spiral can be expressed in terms of its geometrical parameters using (3.1), (3.3), (3.28) and (3.29) as: 


$$
Q=\frac{\pi f_{\mathrm{r}} \mu_{\mathrm{o}} N^{2}\left(\frac{d_{\text {in }}+d_{\text {out }}}{2}\right) c_{1}\left[\ln \left(\frac{c_{2}}{\alpha}\right)+c_{3} \alpha+c_{4} \alpha^{2}\right]}{\frac{\ell_{\text {tot }}}{4 \sqrt{W T}} \sqrt{\pi \mu_{\mathrm{o}} \rho f_{\mathrm{r}}}+31200\left(\frac{f_{\mathrm{r}}}{c}\right)^{4}\left(\sum_{i=1}^{N} d_{i}^{2}\right)^{2}}
$$

The maximum possible Q-factor, $Q_{\max }$, of a spiral and the frequency, $f_{\max }$, where $Q_{\max }$ occurs, can be derived from (3.30) using standard calculus as:

$$
\begin{gathered}
f_{\max }=120.44 \times 10^{6}\left[\frac{\ell_{\text {tot }} \sqrt{\mu_{\mathrm{o}} \rho}}{\sqrt{W T}\left(\sum_{i=1}^{N} d_{i}^{2}\right)^{2}}\right]^{2 / 7} \\
Q_{\max }=\frac{\pi f_{\max } \mu_{0} N^{2}\left(\frac{d_{\text {in }}+d_{\text {out }}}{2}\right) c_{1}\left[\ln \left(\frac{c_{2}}{\alpha}\right)+c_{3} \alpha+c_{4} \alpha^{2}\right]}{\frac{\ell_{\text {tot }}}{4 \sqrt{W T}} \sqrt{\pi \mu_{\mathrm{o}} \rho f_{\text {max }}}+31200\left(\frac{f_{\text {max }}}{c}\right)^{4}\left(\sum_{i=1}^{N} d_{i}^{2}\right)^{2}}
\end{gathered}
$$

Equations (3.31) and (3.32) were derived assuming the proximity effect is negligible; therefore, they are valid only when $S \geq 5 \mathrm{~W}$. A similar work was done in [106] with spirals for resonant inductive coupling and not SCMR, in which both the proximity and radiation resistance effects are ignored.

SCMR requires that each of the TX and RX spiral elements exhibit maximum Qfactor at a frequency $f_{\max }$ that is equal to their resonant frequency $f_{\mathrm{r}}$, in order to achieve maximum power transfer efficiency (i.e., $f_{\mathrm{r}}=f_{\max }$ ). This condition may not be naturally satisfied. This means that if we use (3.31) to design an SCMR system with spirals that have a certain $f_{\max }$ that does not necessarily mean that the spirals will also resonate at $f_{\max }$. If $f_{\mathrm{r}}$ and $f_{\max }$ happens to be different that would mean that the spirals are not resonating at the maximum Q-factor frequency thereby reducing the efficiency of the SCMR system. In what follows, we examine under which conditions $f_{\mathrm{r}}$ and $f_{\max }$ of a spiral are equal. This 
cannot be done analytically, i.e., solving system of equations (3.30) and (3.31) assuming $f_{\mathrm{r}}=f_{\max }$, as there is no adequately accurate analytical formulas for the capacitance of a spiral. Therefore, we perform our analysis using simulations. We used circuit parameter extraction to calculate the $\mathrm{L}, \mathrm{C}$ and $\mathrm{R}$ of the equivalent circuit of a spiral versus frequency using Ansoft Designer thereby allowing us to calculate and compare $f_{\mathrm{r}}$ and $f_{\max }$.

Figure 3.9 shows the $f_{\mathrm{r}}$ and $f_{\max }$ of a spiral with parameters $W=2 \mathrm{~mm}, S=2 \mathrm{~mm}$, $T=2 \mathrm{~mm}$, and $d_{\text {out }}=50 \mathrm{~mm}$ versus the number of turns. The Figure 3.9 shows that as the number of turns of the spiral increases, $f_{\mathrm{r}}$ converges to $f_{\max }$. This happens because: (1) $f_{\max }$ does not change significantly for varying $N$, and (2) the inductance, $L$, and capacitance, $C$, of a spiral increase when $N$ increases as $f_{\mathrm{r}}$ decreases according to (3.1). A simulation study was conducted for several combinations of spiral dimensions within the range of $d_{\text {out }}=50 \mathrm{~mm}$ to $100 \mathrm{~mm}$. Our results show that $f_{\mathrm{r}} \approx f_{\max }$ within a tolerance of $5 \%$ when the following conditions are satisfied:

$$
\begin{gathered}
K \leq 0.1 d_{\text {out }} \\
N=N_{\text {max }}=\frac{d_{\text {out }}}{2 K}
\end{gathered}
$$

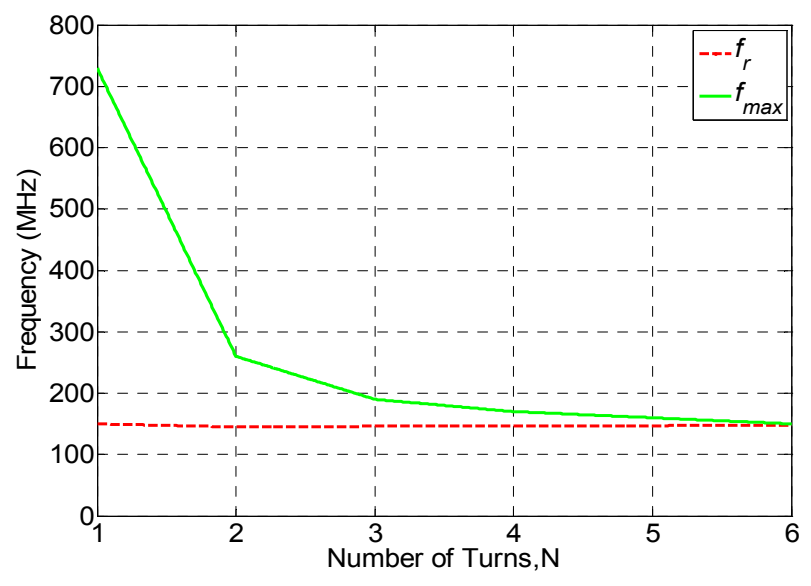

Figure 3.9: Frequencies $f_{\mathrm{r}}$ and $f_{\max }$ of a spiral versus $N$. 
Table 3.2 shows a sample of our results for spirals with geometrical parameters $d_{\text {out }}, N, K, W$ and $T$ that satisfy conditions (3.33) and (3.34). The rightmost column shows the difference between $f_{\mathrm{r}}$ and $f_{\max }$ that is less than $5 \%$ for all cases. Next we examine, if the Q-factor of a spiral has also a global maximum, $Q_{\mathrm{Gmax}}$ in respect to $W$.

Table $3.2 f_{\mathrm{r}}$ and $f_{\max }$ of different spiral dimensions.

\begin{tabular}{|c|c|c|c|c|c|c|c|}
\hline$d_{\text {out }}(\mathrm{mm})$ & $\mathrm{N}$ & $\begin{array}{c}K \\
(\mathrm{~mm})\end{array}$ & $\begin{array}{c}W \\
(\mathrm{~mm})\end{array}$ & $\begin{array}{c}T \\
(\mathrm{~mm})\end{array}$ & $\begin{array}{c}f_{\mathrm{r}} \\
(\mathrm{MHz})\end{array}$ & $\begin{array}{c}f_{\max } \\
(\mathrm{MHz})\end{array}$ & $\begin{array}{c}\text { Diff. } \\
\%\end{array}$ \\
\hline 50 & 8 & 3 & 2 & 2.0 & 129.35 & 124.20 & 4.2 \\
\hline 50 & 12 & 2.1 & 1 & 0.5 & 93.60 & 96.07 & 2.6 \\
\hline 50 & 5 & 5 & 2 & 2.0 & 220.13 & 215.60 & 2.1 \\
\hline 75 & 15 & 2.5 & 1 & 1.0 & 50.58 & 49.00 & 3.2 \\
\hline 75 & 11 & 3.4 & 3 & 2.0 & 61.95 & 62.00 & 0.1 \\
\hline 75 & 7 & 5 & 2 & 0.5 & 102.39 & 98.2 & 4.3 \\
\hline 100 & 19 & 2.6 & 1 & 1.5 & 29.41 & 29.1 & 1.1 \\
\hline 100 & 14 & 3.5 & 2 & 1.0 & 39.81 & 40.6 & 2.0 \\
\hline 100 & 10 & 5 & 3.5 & 0.5 & 57.43 & 57.7 & 0.5 \\
\hline
\end{tabular}

The proximity effect should also be considered as we increase the width, by including the factor $R_{\mathrm{p}} / R_{\mathrm{o}},(3.32)$ can be written as:

$$
Q_{\text {max }}=\frac{\pi f_{\text {max }} \mu_{\mathrm{o}} N^{2}\left(\frac{d_{\text {in }}+d_{\text {out }}}{2}\right) c_{1}\left[\ln \left(\frac{c_{2}}{\alpha}\right)+c_{3} \alpha+c_{4} \alpha^{2}\right]}{\frac{\ell_{\text {tot }}}{4 \sqrt{W T}} \sqrt{\pi \mu_{\mathrm{o}} \rho f_{\text {max }}}\left(1+\frac{R_{\mathrm{P}}}{R_{\mathrm{o}}}\right)+31200\left(\frac{f_{\text {max }}}{c}\right)^{4}\left(\sum_{i=1}^{N} d_{i}^{2}\right)^{2}}
$$

The standard calculus cannot derive the global maximum analytically due to the complexity of (3.35). Nevertheless, $Q_{\mathrm{Gmax}}$ can be calculated analytically by plotting $Q_{\max }$ using (3.35) and observing if a global maximum exists. For example, a spiral with parameters $N=5, K=W+S=10.2 \mathrm{~mm}, T=0.5 \mathrm{~mm}, d_{\text {out }}=100 \mathrm{~mm}$ is examined. The maximum Q-factor $Q_{\max }$ is calculated analytically using (3.35) for $W$ varying from 0.2 $\mathrm{mm}$ to $9 \mathrm{~mm}$ while keeping the distance between the centers of adjacent turns $(K)$ of the 
spiral constant at $10.2 \mathrm{~mm}$. Figure 3.10 shows the plots of $Q_{\max }$ versus $W$ and compares the analytical calculations with simulations. Figure 3.10 also illustrates clearly that a global $Q_{\text {Gmax }}$ occurs at $W=3.6 \mathrm{~mm}$, in both the analytical and simulation results. This indicates that the spiral designed with a width of $3.6 \mathrm{~mm}$ will be globally optimum and have maximum efficiency for: $N=5, T=0.5 \mathrm{~mm}$ and $d_{\text {out }}=100 \mathrm{~mm}$ and $K=10.2 \mathrm{~mm}$. The existence of the global maximum can be explained with reference to (3.27). The ohmic resistance of the spiral is inversely proportional to $\sqrt{W}$ and it decreases if the width of the spiral is increased. However, if the width is increased while keeping $K$ constant, the spacing between the turns decreases thereby increasing the proximity effect factor $R_{\mathrm{p}} / R_{\mathrm{o}}$. Hence, $R_{\mathrm{p}} / R_{\mathrm{o}}$ sets a limit on the minimum value that the ohmic resistance can attain. The width corresponding to the minimum resistance is its optimum value, and when this happens, $Q_{\max }$ can attain its global maximum.

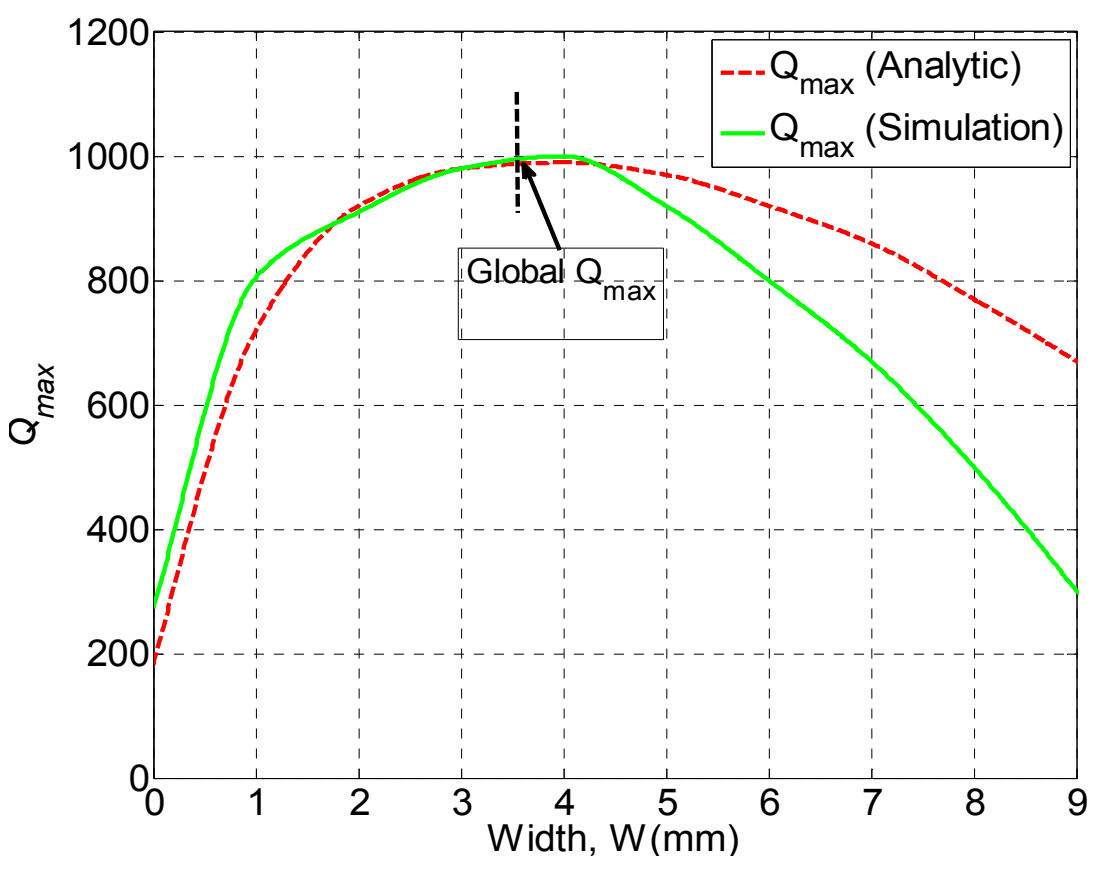

Figure 3.10: Local $Q_{\max }$ Vs. W. 
In order to verify that $Q_{\text {Gmax }}$ exists, we designed SCMR systems that utilized the spiral parameters: $N=5, K=10.2 \mathrm{~mm}, T=0.5 \mathrm{~mm}, d_{\text {out }}=100 \mathrm{~mm}$ and $W=0.2 \mathrm{~mm}$, $3.6 \mathrm{~mm}$ and $9 \mathrm{~mm}$. The distance between TX and RX resonators was set to $l_{2}=150 \mathrm{~mm}$. The efficiency versus frequency plot of each of these designs is shown in Figure 3.11, which illustrates that the SCMR system with the highest efficiency is the one that uses a spiral with $W=3.6 \mathrm{~mm}$. In addition, Figure 3.12 plots the maximum efficiency achieved by the SCMR systems for $W$ varying from $0.2 \mathrm{~mm}$ to $9 \mathrm{~mm}$. Figure 3.13 plots the frequency where the maximum efficiency, $f_{\text {emax, }}$ occurs for each of the SCMR systems where $W$ is varying and $N, K, T$ and $d_{\text {out }}$ are kept constant. It can be seen that $f_{\text {emax }}$ does not significantly change for varying $W$. In fact, the maximum variation in $f_{\text {emax }}$ is only $4.38 \%$ across the entire range of $W$. It can be concluded that the selection of the right value for $W$ is critical in order to achieve globally maximum efficiency.

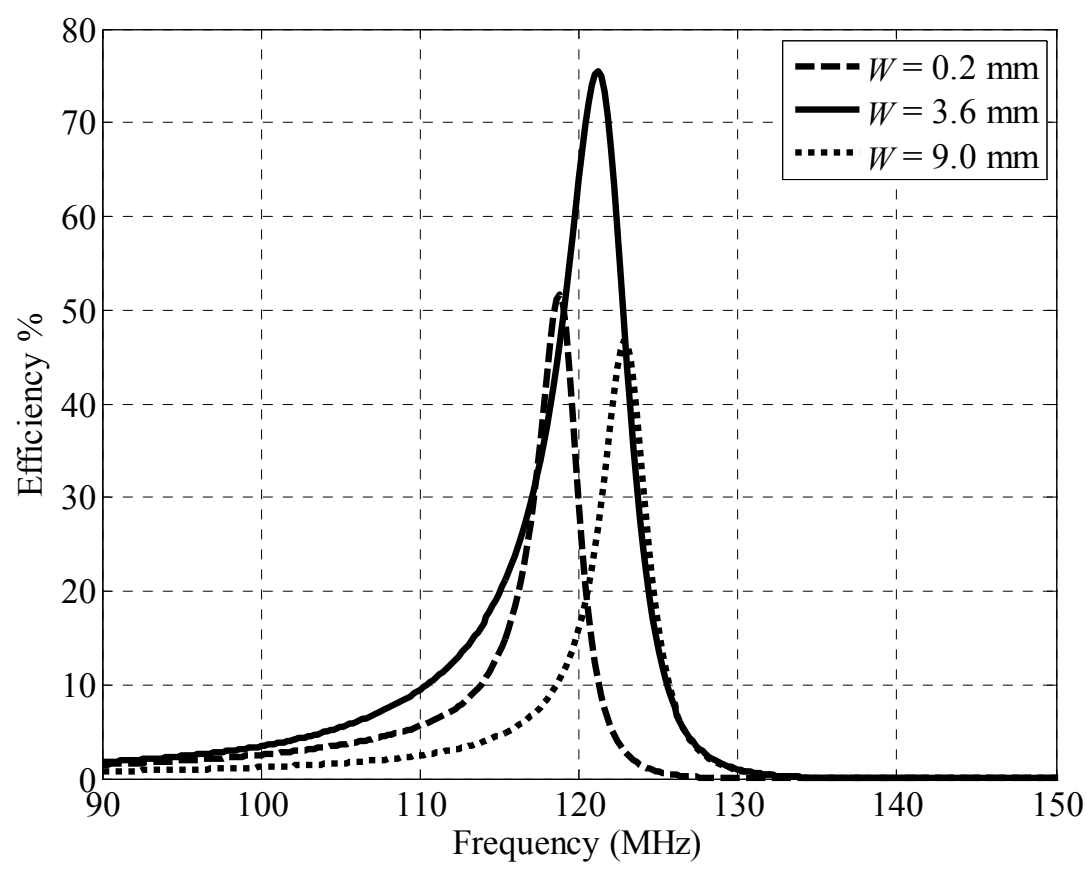

Figure 3.11: The efficiency of the SCMR system for $W=0.2,3.6$ and $9.0 \mathrm{~mm}$. 


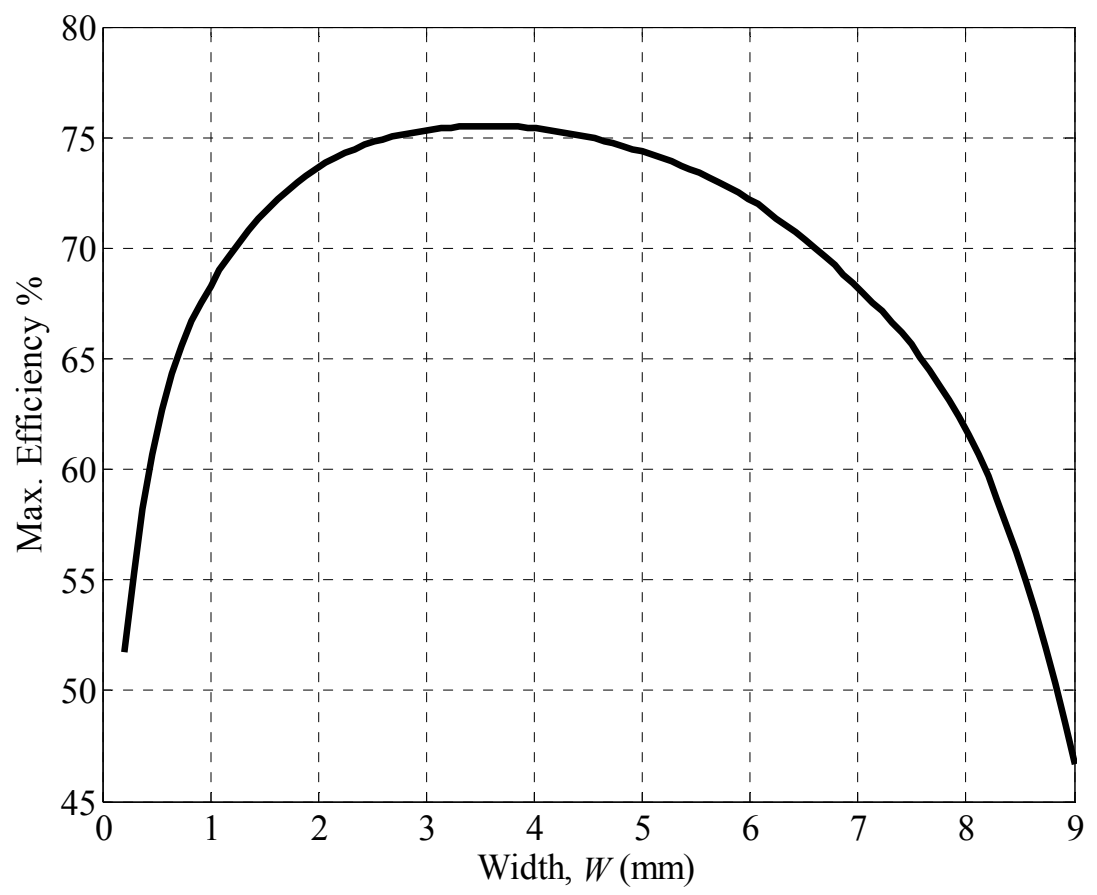

Figure 3.12: The maximum efficiency of the SCMR system versus $W$.

Based on the results so far, we can propose a process of designing spirals for globally optimal SCMR systems with maximum efficiency as follows: (1) pick desired frequency, $f_{\mathrm{o}}$, for WPT, (2) design spiral using (3. 31) and satisfying $S>5 \mathrm{~W}$ to exhibit maximum Q-factor at $f_{\mathrm{o}},(3)$ use (3.35) to find the optimum cross-sectional width of a spiral, $W$; (4) model SCMR system with the designed spirals in simulation software using optimal $W$; (5) fine tune the performance of SCMR design and $f_{\text {emax }}$ in simulation software (e.g., by making minor adjustment in K). 


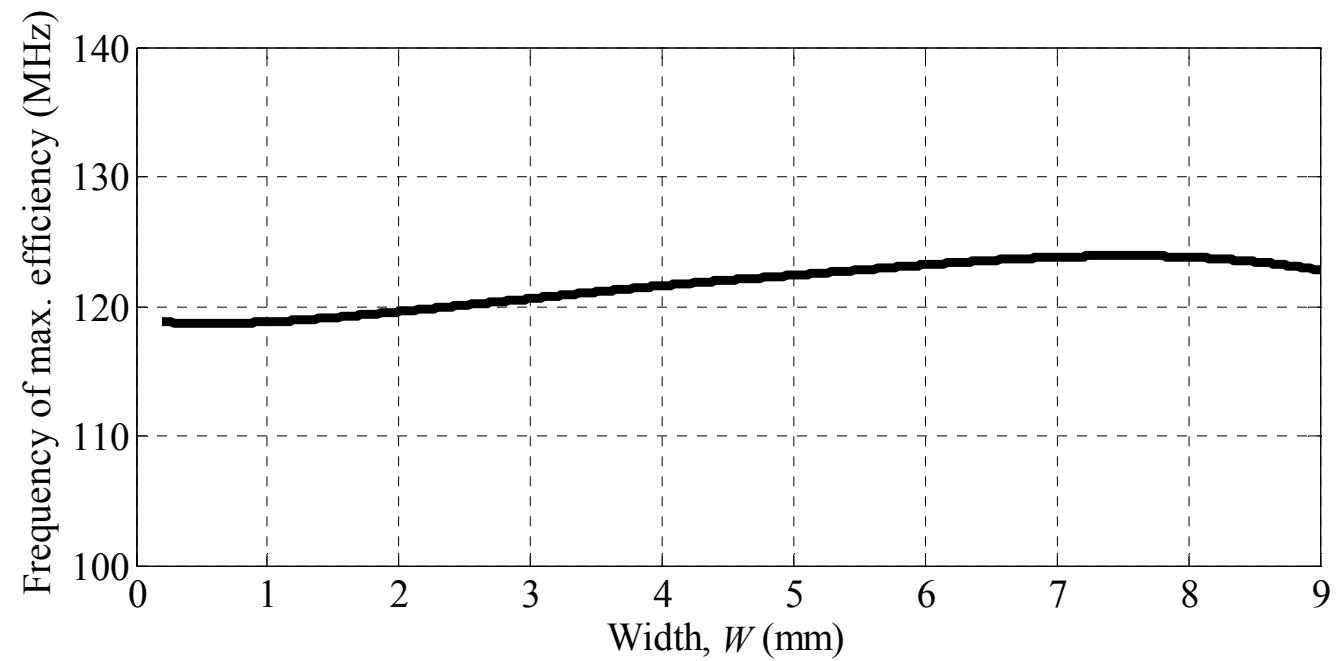

Figure 3.13: The frequency of maximum efficiency versus width of the spiral, $W$.

\subsection{Summary}

This chapter analytically examines the optimal design of SCMR elements. Specifically, loops, helices and spirals were analyzed. In these cases, we analytically derived equations that can be used to design optimal SCMR systems. In addition, our formulations proved that the global maximum Q-factor of a loop or helix is achieved when the ratio between the radius, $r$, and the cross-sectional radius, $r_{c}$, of the loop is approximately equal to 9.52 . This important finding can be used to design maximally efficient SCMR wireless powering systems, that use loops or helices as transmitting and receiving elements. Also, this chapter examines the optimal design of SCMR systems that use spiral resonators. Specifically, a methodology, which guarantees globally optimal spiral based SCMR systems, has been derived and verified. 


\section{CHAPTER 4}

\section{WIRELESS POWER TRANSFER IN CONCRETE STRUCTURES}

Transmission of RF energy through plain and reinforced concrete via SCMR are analyzed in this chapter. The Electromagnetic properties of the concrete are modeled using the extended Debye model at different depths and the properties of the concrete for six different humidity levels. Power was scavenged from bridge vibrations in [107], while an air core coil was connected to a voltage doubler to collect power in [108]. RFID technology was employed in [109], [110] to transfer power by inductive coupling between master and sensor magnetic coils. In addition, the effects of concrete material properties of the radiation pattern and gain of a microstrip patch antenna were studied in [111].

Health monitoring of structures is a very important topic that has been studied extensively. Continuous monitoring can identify potential problems or hazards and provide essential warnings. Such warnings can allow engineers to intervene and correct dangerous structural problems. Structural health monitoring is commonly performed with sensors that are embedded in concrete. These sensors can measure volumetric data such as, temperature, displacement, pressure, strain, and humidity. The data can then be used to access the health of a structure during and/or after construction and detect problems such as cracking and rebar corrosion.

Most monitoring systems rely on embedded sensors connected through wires to a central station, which provides them with power and collects their data. The installation of such sensors is usually expensive, inefficient, labor intensive and difficult. In fact, the installation of a monitoring system can represent up to $25 \%$ of the total system cost with 
over $75 \%$ of the installation time focused solely on the installation of wires [1], [112] Also, if the wires of certain sensors get corroded then these sensors become useless.

In this chapter, we use a computational methodology to develop an optimal receiver/transmitter system that will support efficient wireless power transfer from air to concrete at different humidity levels. The material properties of concrete vary with the frequency and humidity level, and they are modeled using the extended Debye model [113], [114]. The performance of SCMR wireless power transfer systems is analyzed using coupling simulations for concrete with different humidity levels and at various depths. In addition, the simulation results are compared with measurements.

\subsection{Simulation Models}

The transmission of the electromagnetic field in plain and reinforced concrete has been studied before using the finite element method (FEM), finite-difference timedomain (FDTD) technique, and the method of moments (MoM). The propagation losses in brick and concrete walls were studied at $900 \mathrm{MHz}$ band using measurements and MoM simulations in [115]. Optimal frequencies for propagation in plain and reinforced concrete slab were identified in [116] for far field applications for various concrete thicknesses, rebar diameters and incident angles. This work is focused on studying the optimal power transfer from air to plain and reinforced concrete via SCMR, for various humidity levels of the concrete, concrete slab thicknesses, rebars period, rebar sizes. 


\subsubsection{The Plain and Reinforced Concrete Models}

The plain concrete slab model is 8 " thick, 12 " wide and it is placed in the bottom of the model, with air box added above the slab. Previous work in [116] used fixed concrete permittivity and conductivity values. The reinforced bar in the simulations has these parameters: mesh period of 6", rebar diameter of (5/8)" and the gap between two rebar layers of 3.5" (see Figure 4.1). The material of the reinforced bars is steel in HFSS. Our work implements the extended Debye model the simulations in order to accurately describe the electromagnetic properties of concrete at different humidity conditions and frequencies.

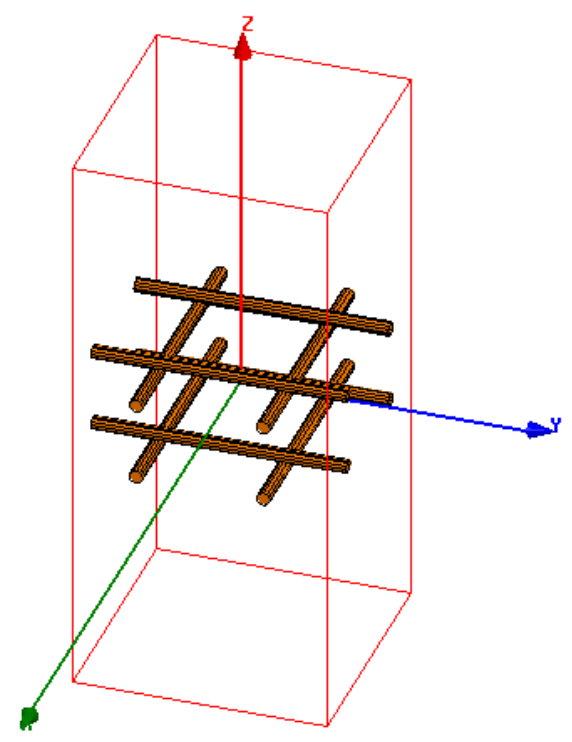

Figure 4.1: Two layers of cross rebars in HFSS.

In Figure 4.2, all parameters are kept the same except the mesh period, $g$, which is increased to 12 " in order to examine the effects of various mesh periods on power transmission. 


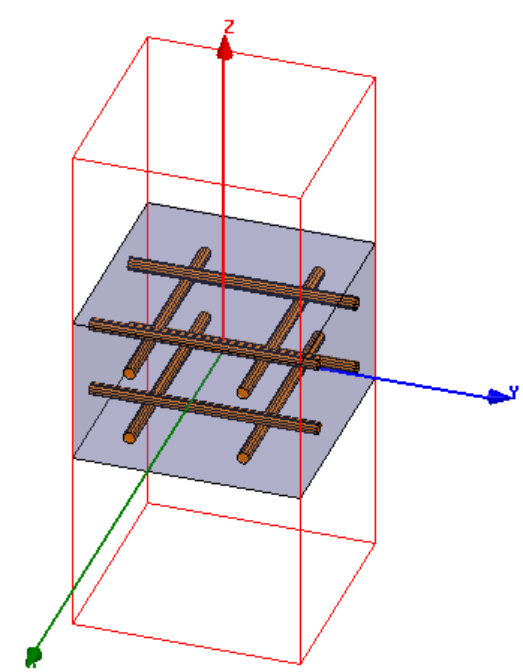

(a) Rebar period is 6".

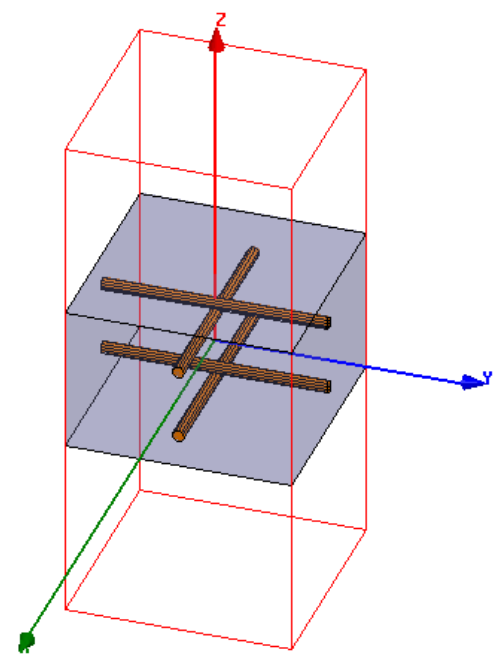

(b) Rebar period is 12 ".

Figure 4.2: Reinforced concrete simulation setup in HFSS.

\subsection{Optimization of Wireless Power Transfer in Concrete}

Prior to designing the WPT system for air-concrete interface, first we outline our computational methodology for the design and analysis of SCMR systems for air-to-air power transfer. Our computational methodology consists of the following steps:

1. Select a desired resonant frequency, $f_{r}$, at which the TX and RX will operate.

2. Pick the geometric parameters of a loop using the equations (3.1)-(3.6) so that $f_{r}$ is equal to the desired resonant frequency.

3. Simulate the TX loop in Ansoft Designer with geometric parameters obtained from step 2 above, compute the self-inductance and Q-factor as function of frequency. The TX loop is then tuned for maximum Q-factor.

4. Calculate the required capacitance that needs to be connected to the loop in order to exhibit a resonance frequency $f_{r}$, using (1). 
5. Add an identical loop at a distance of $\ell_{2}$ which is the RX loop. Then, simulate both TX and RX loops using ansoft designer in order to calculate their corresponding self inductance and Q factor .

6. Recalculate the required capacitances that need to be connected to the TX and RX loops, so that they both are resonant at frequency $f_{r}$.

7. Add the source and load loops at distances $\ell_{1}=\ell_{3}=10 \mathrm{~cm}$ and $\ell_{2}=20 \mathrm{~cm}$.

8. From the simulation results of step 7, we export the equivalent four port network into Ansoft Nexxim, and connect the required capacitors (already calculated from step 6) to the TX and RX resonator loop and terminate the load loop in a load.

9. Perform Nexxim simulation of the circuit for the frequency range of interest, in order to calculate the power transferred.

10. Fine tune the capacitors that are connected to TX and RX loops in order to maximize the power delivered to the load.

11. Calculate the efficiency for the wireless power transfer using the following formula:

$$
\eta=\frac{\text { Power delivered to the load }}{\text { Power from source }}=\frac{\left|S_{21}\right|^{2}}{\left(1-\left|S_{11}\right|^{2}\right)}
$$

A typical reinforced concrete slab is shown in Fig 4.2. It consists of two rebar layers inside the concrete slab, and it is widely used in concrete structures, such as, reinforced concrete walls and bridge decks. Reinforced bars are metallic periodic structures that are expected to significantly affect the propagation of EM waves when they are inserted into concrete structures. Therefore, rigorous analysis of rebar effects is necessary for the development of optimum wireless powering systems. Here WPT from air to concrete is studied for various SCMR elements, such as loops, helices and spirals at 
different various humidity conditions and depths, in plain and reinforced concrete structures via SCMR.

\subsection{Wireless Power Transfer with Loops}

An SCMR designed for the air-to-air power transfer cannot be directly used in nonhomogenous interfaces (e.g., from air to the concrete) and must be modified. The electrical performance of each loop depends on the material properties of the medium around it. Therefore, if the RX loop that was designed for the air-to-air transmission is embedded in concrete without any change, it will exhibit a Q-factor that is not maximum at the desired resonant frequency thereby significantly reducing the SCMR system's efficiency. In order to illustrate this point, the same pair of RX and load loops that were designed for air $(r=10 \mathrm{~cm}, a=2.2 \mathrm{~mm})$ is embedded in concrete as shown in Figure 4.3, where $H$ is the placement height of TX loop above air-to-concrete interface, $D$ is the placement depth of RX loop in concrete, and $h$ is the humidity level of the concrete. We simulated the system shown in Figure 4.3 using Ansoft Designer and calculated the Qfactors for TX and RX loops with $H=10 \mathrm{~cm}, D=4 \mathrm{~cm}$, and $h=0.2 \%$.

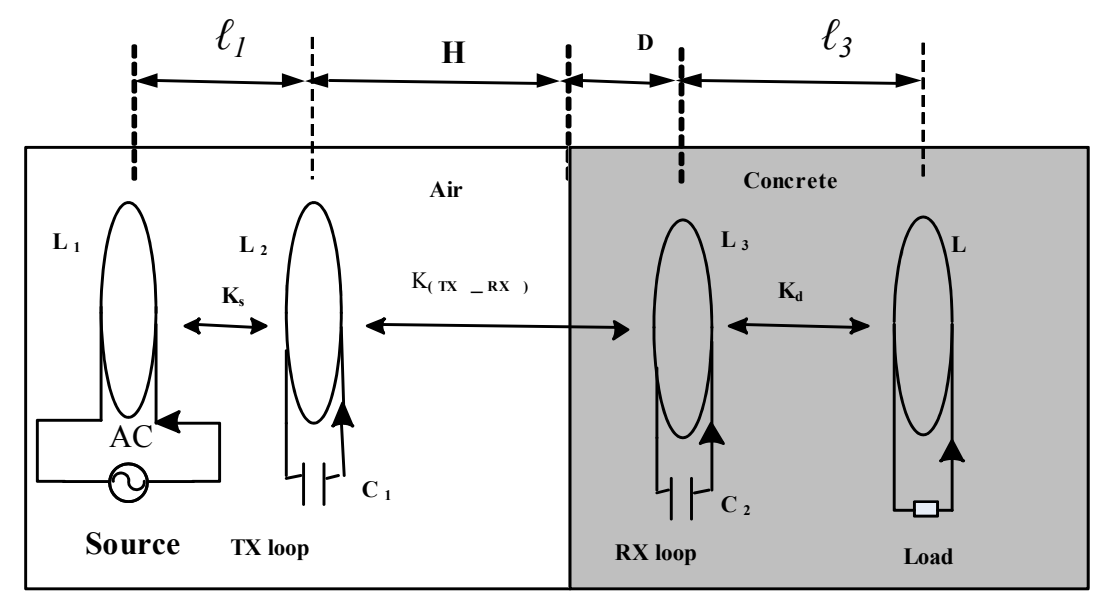

Figure 4.3: An SCMR system for air-to-concrete transmission. 
The dispersive properties of concrete are modeled using the extended Debye model described in [113], [114]. Also, the properties of concrete depend on the humidity level. The humidity condition describes the percentage of water by volume, and it is represented here by the symbol, $h$. The Debye model parameter values for six different humidity conditions are shown in Table 4.1 , where $\varepsilon_{\text {static }}$ and $\varepsilon_{\infty}$ are the relative permittivities at low and high frequencies, respectively, $\tau$ is the relaxation time, and $\sigma_{d c}$ is the DC electrical conductivity of concrete [113]. This Debye model is implemented in Ansoft Designer using the "Debye Model Input" menu in order to accurately model the properties of concrete in our simulations.

Table 4-1. Extended Debye's model parameters values for concrete.

\begin{tabular}{|c|c|c|c|c|}
\hline $\begin{array}{c}\text { Moisture } \\
\text { content }\end{array}$ & $\varepsilon_{\text {Static }}$ & $\varepsilon_{\infty}$ & $\tau(n s)$ & $\sigma_{d c}\left(\Omega^{1} m^{-1}\right)$ \\
\hline $0.2 \%$ & 4.814 & 4.507 & 0.82 & $6.06 \times 10^{-4}$ \\
\hline $2.8 \%$ & 6.750 & 5.503 & 2.28 & $2.03 \times 10^{-3}$ \\
\hline $5.5 \%$ & 8.630 & 6.023 & 1.00 & $5.15 \times 10^{-3}$ \\
\hline $6.2 \%$ & 9.140 & 5.930 & 0.80 & $6.7 \times 10^{-3}$ \\
\hline $9.3 \%$ & 11.19 & 7.200 & 0.73 & $23 \times 10^{-3}$ \\
\hline $12 \%$ & 12.84 & 7.420 & 0.611 & $20.6 \times 10^{-3}$ \\
\hline
\end{tabular}

By comparing the TX and RX loop Q-factors for the air-to-air and the air-toconcrete power transfer systems, we observe that the RX loop's Q-factor is significantly different for these two scenarios. This change occurs due to the higher conductivity and dielectric constant of concrete compared to the ones of air. Specifically, the RX loop in air exhibits maximum Q-factor of 1300 at $39.75 \mathrm{MHz}$, whereas the identical RX loop embedded in concrete has a maximum Q-factor of 380 at $5.1 \mathrm{MHz}$. Therefore, the resonant frequencies of TX and RX loops are different, which in turn significantly 
reduces the SCMR system's wireless efficiency. This problem needs to be addressed by redesigning the RX loop such that its Q-factor is maximum at the desired resonant frequency, $f_{r}$, which must be the same with the resonant frequency of the TX loop. Also, in order to achieve maximum power transfer from the source to load, the distance between TX loop and source loop, $\ell_{1}$, and the distance between RX loop and load loop elements, $\ell_{3}$, must be optimized. Tables 4-2 and 4-3 illustrate the effects of varying distances $\ell_{1}$ and $\ell_{3}$, respectively for the SCMR system of Figure 4.3. The results show that maximum power transfer is achieved, when $\ell_{1}=\ell_{3}=4 \mathrm{~cm}$ (these distances will be used for the SCMR designs considered below).

Table 4-2. Comparison of efficiencies for varying $\ell_{1}\left(H=10 \mathrm{~cm}, D=10 \mathrm{~cm}, \ell_{3}=4 \mathrm{~cm}\right)$

\begin{tabular}{|c|c|c|c|c|c|}
\hline$\ell_{1}(\mathrm{~cm})$ & 1 & 2 & 3 & 4 & 5 \\
\hline $\begin{array}{c}\text { Efficiency } \\
(\%)\end{array}$ & 28.1 & 54.3 & 57.9 & 59.2 & 56.4 \\
\hline
\end{tabular}

Table 4-3. Comparison of efficiencies $\ell_{3}\left(H=10 \mathrm{~cm}, D=10 \mathrm{~cm}, \ell_{3}=4 \mathrm{~cm}\right)$

\begin{tabular}{|c|c|c|c|c|c|}
\hline$\ell_{3}(\mathrm{~cm})$ & 1 & 2 & 3 & 4 & 5 \\
\hline $\begin{array}{c}\text { Efficiency } \\
(\%)\end{array}$ & 28.3 & 55.0 & 57.8 & 59.9 & 57 \\
\hline
\end{tabular}

Furthermore, in order to redesign the embedded RX loop in concrete for efficient power transfer we follow the steps below:

12. Enclose RX and load loops in a $7.5 \times 7.5 \times 3 \mathrm{~cm}^{3}$ cubic air box as shown in Figure 4.4 An air-box is used in order to maximize the value of the RX loop's Q-factor. This is because we discovered through simulation analysis that when the RX and load loops are inside an air-box instead of touching the concrete, both the Q-factor and the efficiency improve significantly. Similar configuration to our air-box was used by [52], [53], where 
an air-gap was added on top of patch antennas embedded in concrete in order to optimize their performance. In practice, the air-box around the RX and load loops can be incorporated by the packaging of sensors. Such packaging must be used in order to protect the circuitry of sensors inside concrete. Therefore, since the height of our design's air-box is only $3 \mathrm{~cm}$, it can be easily realized by the packaging of our design.

13. Make use of (3.1) to (3.5) to design and optimize the RX loop and also calculate the required capacitance. The optimized RX loop's size was reduced to $3.5 \mathrm{~cm}$ radius with a cross-sectional radius of $2.2 \mathrm{~mm}$. The resonating capacitances were calculated to be $C_{l}=$ $38.4 \mathrm{pF}$ and $C_{2}=0.137 \mathrm{nF}$. Also, the distances are $\ell_{1}=4 \mathrm{~cm}, \ell_{2}=H+D=20 \mathrm{~cm}(H=10$ $\mathrm{cm}$ and $D=10 \mathrm{~cm}$ ), and $\ell_{3}=2 \mathrm{~cm}$. The Q-factors of the TX and RX loops are maximum Q-factor at the same frequency.

14. Simulate the model from step (13) in Ansoft Nexxim and calculate the efficiency.

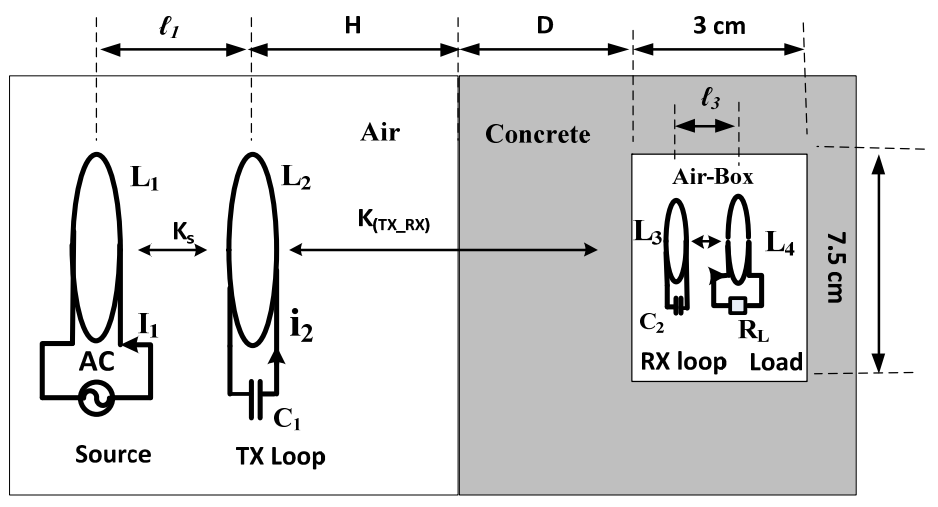

Figure 4.4: An SCMR system for air-to-concrete transmission.

Steps (12) to (14) lead to a design where the maximum Q-factor frequencies of the RX and TX loops (RX loop is embedded in concrete) are equal to $39.75 \mathrm{MHz}$. Also, the Q-factor of the redesigned RX loop at $39.75 \mathrm{MHz}$ is 920 . This design of Figure 4.4 achieved a wireless power transfer (from source to load) efficiency of $59.0 \%$ for a 
concrete humidity of $\mathrm{h}=0.2 \%$. These results clearly illustrate that SCMR systems can be designed to provide wireless powering efficiencies through non-homogeneous interfaces that are significantly larger than the efficiencies achieved by other conventional wireless powering methods [77], [78]. In what follows, a detailed study of the effects of the height, $H$, the depth, $D$, and the humidity, $h$, on the efficiency of such SCMR systems is presented.

\subsubsection{Effects of varying TX loop's Placement Height}

In this section, the height, $H$, is varied between $10 \mathrm{~cm}$ and $50 \mathrm{~cm}$ and the depth, $D$ is fixed at $10 \mathrm{~cm}$ (see Figure 4.5).

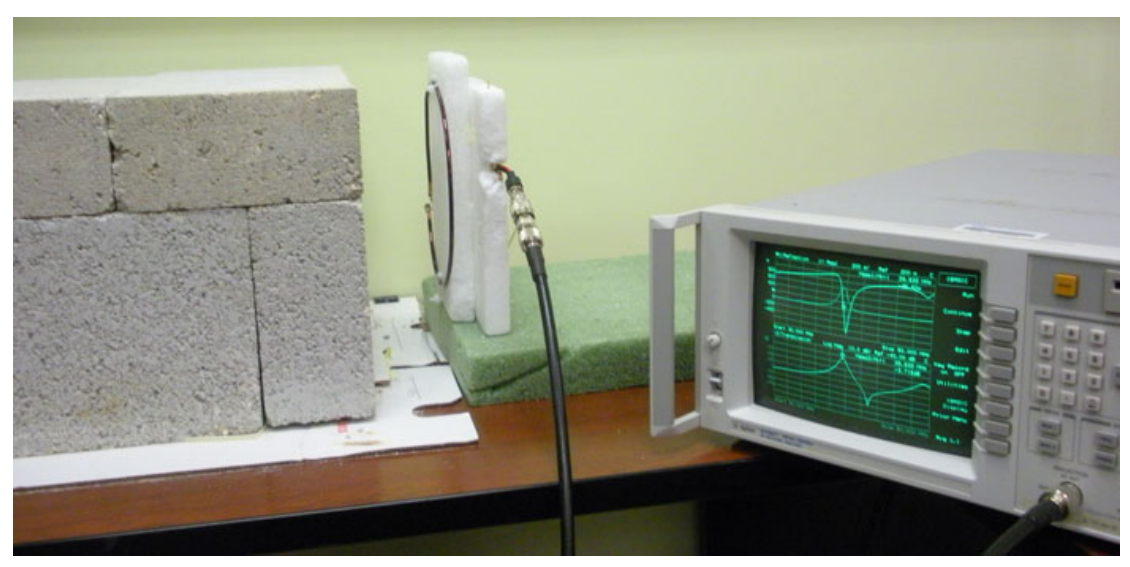

Figure 4.5: Measurement setup of an SCMR system for air-to-concrete interface.

The setup of our measurements is shown in Figure 4.5. Specifically, an HP 8714 network analyzer was used for all measurements. As part of the concrete setup, we utilized a concrete block to simulate as closely as possible the air-box around the SCMR's receiver. Figure 4.6 shows the simulated and measured efficiency of the SCMR system of Figure 4.4 for different values of $H(H=10,30$ or $50 \mathrm{~cm})$. All simulations 
were carried out using Ansoft Designer and HFSS; Ansoft Designer was used for the large number of Q-factor calculations to save computational time, as it is faster than Ansoft HFSS, and the efficiency analysis was performed with Ansoft HFSS. The simulation results agree very well with the measurements (see Figure 4.6). The maximum measured efficiency of $57.2 \%$ was achieved for $H=10 \mathrm{~cm}(59.0 \%$ in the simulation results). Figure 4.6 shows again that the SCMR method can achieve efficiencies that are significantly larger than the efficiencies that are achieved by conventional inductive coupling techniques. Finally, Figure 4.7 shows the measured efficiency versus height $H$, which peaks when the TX loop is placed at a height of $10 \mathrm{~cm}$ above the air-concrete interface. Therefore, there is an optimum value of TX loop's placement height, $H$, above the air-to-concrete interface for which SCMR's efficiency is maximized.

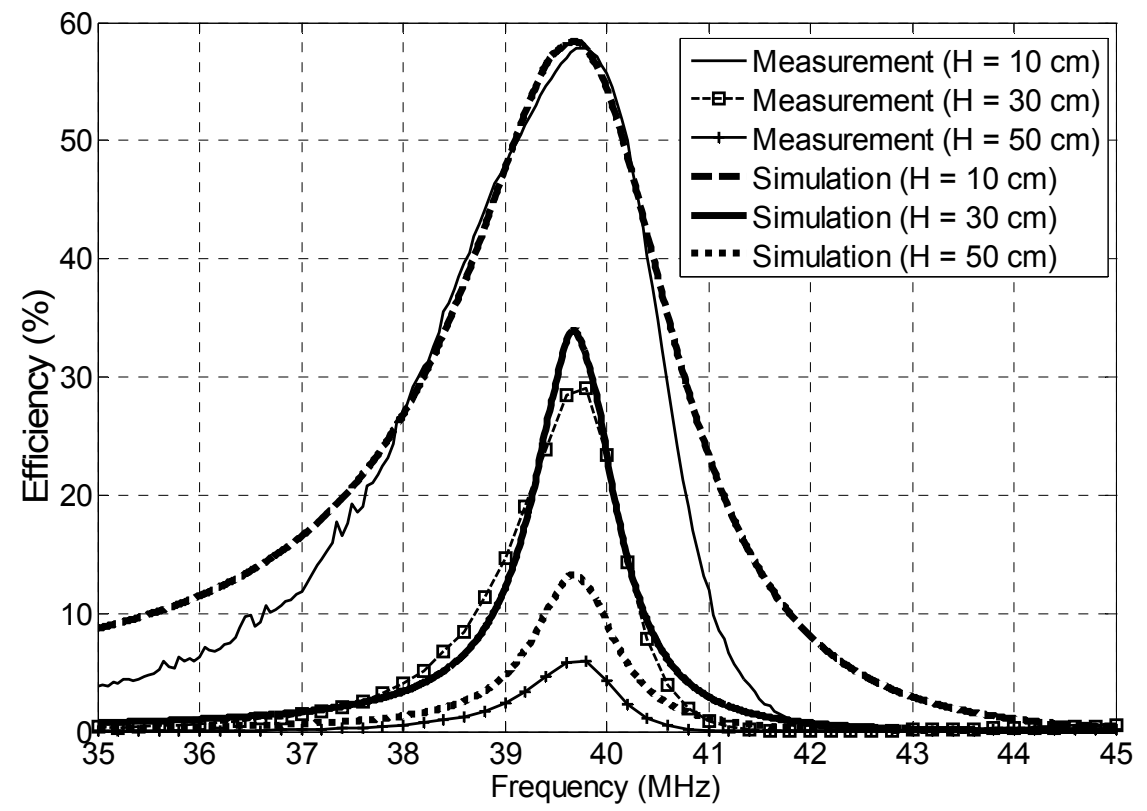

Figure 4.6: Efficiency for various placement heights $(H)$ of the TX loop $(h=0.2 \%)$. 


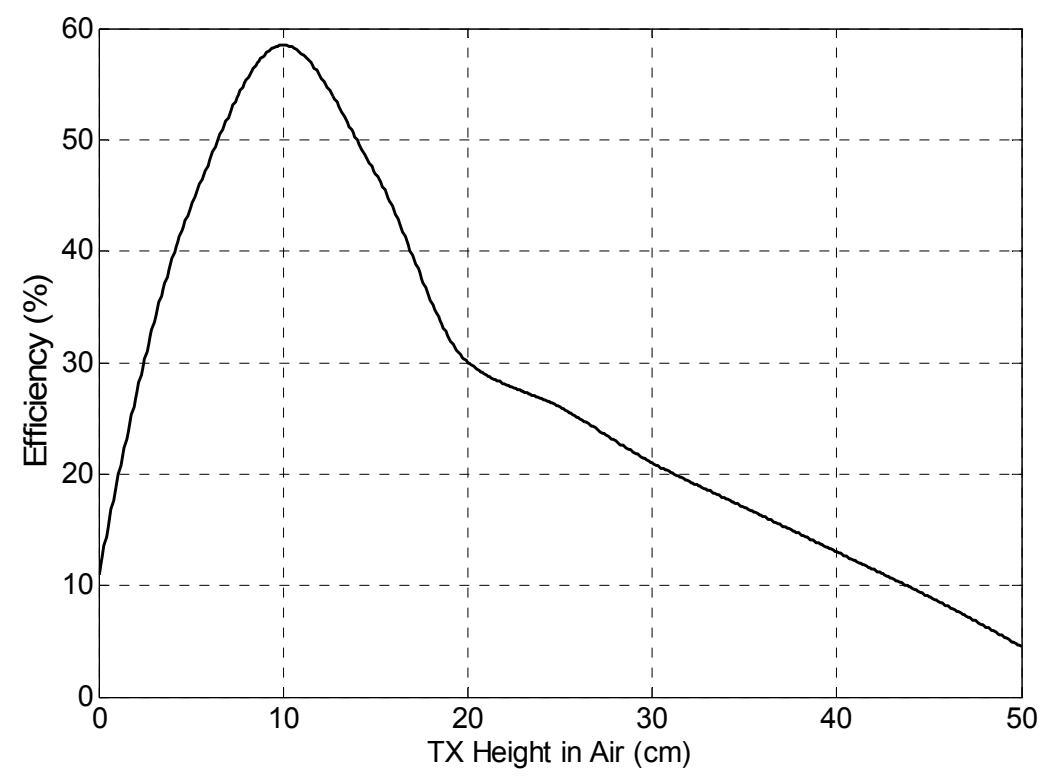

Figure 4.7: Measured efficiency versus the placement height, $H$, of TX loop $(h=0.2 \%)$

This optimum value of $H$ does not coincide with the minimum value of $H$ because when $H$ is too small, coupling effects between the TX and RX loops de-tune the SCMR system thereby reducing its efficiency.

\subsubsection{Effects of varying RX loop's Placement Depth in Concrete}

This section examines the effects of varying depth, $D$, when the height is $H=10$ $\mathrm{cm}$ and concrete's humidity is $h=0.2 \%$ (see Figure 4.4). Our simulation analysis concluded that the depth, $D$, does not significantly affect the Q-factors of both the TX and RX loops. Also, Figure 4.8 illustrates the simulated and measured efficiency for various depths. Figure 4.9 shows the measured efficiency versus the increasing depth, $D$. It can be seen that SCMR's efficiency peaks when the RX loop is placed at a depth of $10 \mathrm{~cm}$. This optimum depth is not the minimum depth because when the depth is too small, coupling effects between the TX and RX loops de-tune the SCMR system thereby 
reducing its efficiency. Therefore, TX loop's placement height, $H$, and RX loop's placement depth, $D$, must be carefully chosen and analyzed in order to achieve optimal efficiencies in practical SCMR system installations.

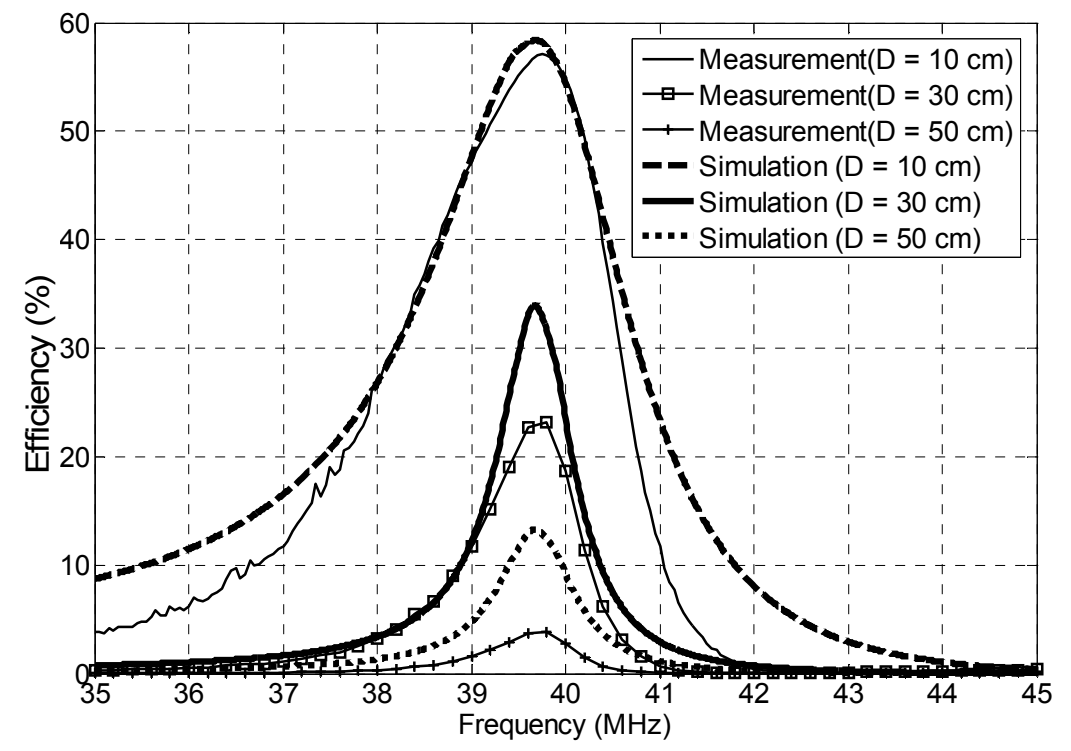

Figure 4.8: Efficiency for various placement depths, $D$, of the RX loop in concrete ( $h=$ $0.2 \%)$.

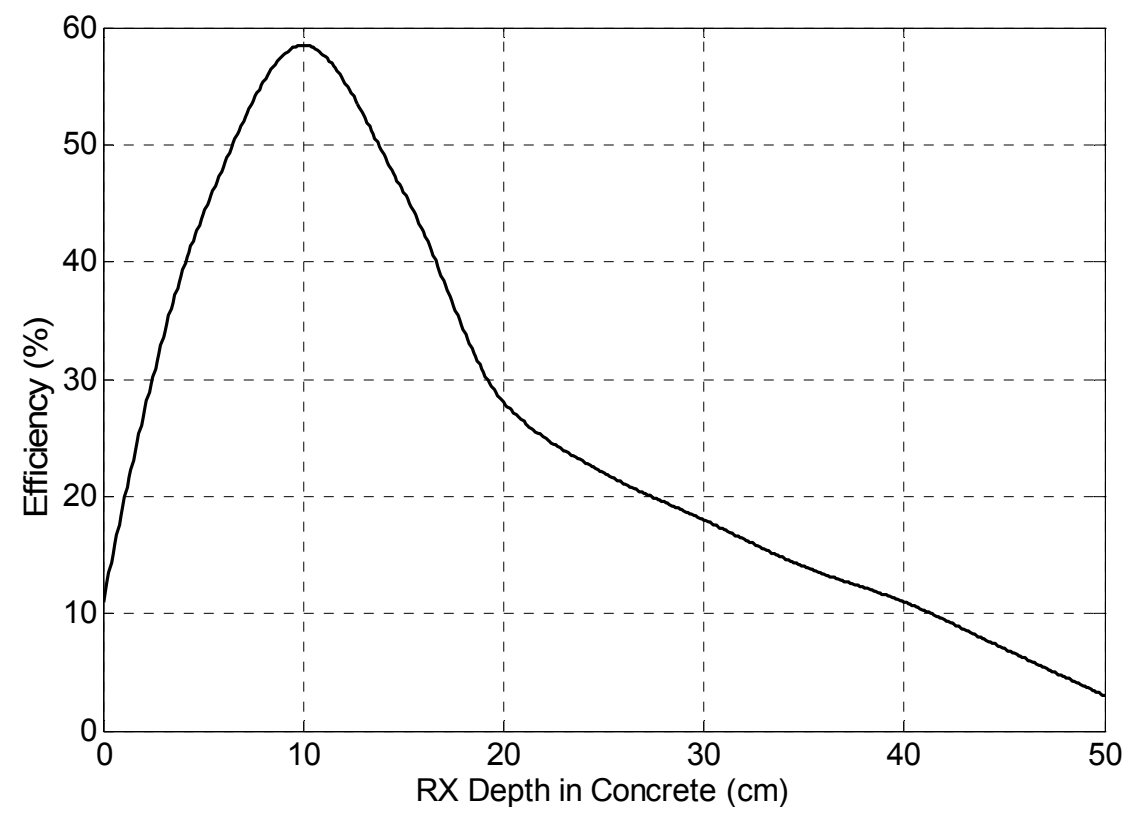

Figure 4.9: Measured efficiency versus the placement depth, $D$, of RX loop $(h=$ $0.2 \%)$. 
Also, by comparing Figs. 4.6 and Figure 4.9, it can be concluded that the efficiency of an SCMR system for air-to-concrete interface drops from its maximum at a slightly faster rate for increasing placement depth rather than increasing placement height.

\subsubsection{Humidity Effects}

Here, the effects of concrete's humidity [105] on the performance of the SCMR system of Figure 4.4 are examined. Specifically, the humidity effects are studied because many applications require monitoring a concrete structure while the concrete is still wet and curing. For example, high strength concrete may take 6 to 60 days to cure depending on the structure and the weather conditions. Therefore, high strength concrete needs to be closely monitored by sensors while it is curing (it is wet) to ensure that it reaches its full strength before any mechanical load is applied to it. Hence, when designing SCMR wireless powering systems for concrete applications, the concrete's moisture content must be taken into consideration to maximize the efficiency.

Here, the Q-factor and the wireless powering efficiency are calculated for six humidity levels for the same SCMR system (see Table 4-4). It can be concluded that the SCMR system's efficiency reduces as the humidity increases. This is attributed to the increasing conductivity of concrete with increasing humidity level that in turn affects SCMR's performance by de-tuning the resonant frequency of RX and load loops inside concrete. 
Table 4-4. Humidity effects on SCMR design $(H=10 \mathrm{~cm}, D=10 \mathrm{~cm})$

\begin{tabular}{|c|c|c|c|c|}
\hline $\begin{array}{c}\text { Humidity } \\
\text { Level (\%) }\end{array}$ & $\begin{array}{c}\text { RX loop's } \\
a(\mathrm{~mm})\end{array}$ & $\begin{array}{c}\text { TX loop Q- } \\
\text { factor }\end{array}$ & $\begin{array}{c}\text { Rx loop Q- } \\
\text { factor }\end{array}$ & $\begin{array}{c}\text { Efficiency } \\
(\%)\end{array}$ \\
\hline 0.2 & 2.2 & 3650 & 2010 & 59.0 \\
\hline 2.8 & 2.2 & 920 & 1269 & 18.5 \\
\hline 5.5 & 2.2 & 900 & 915 & 14.5 \\
\hline 6.2 & 2.2 & 890 & 830 & 13 \\
\hline 9.3 & 2.2 & 750 & 479 & 9.8 \\
\hline 12 & 2.2 & 300 & 432 & 8.7 \\
\hline
\end{tabular}

Subsequently, the RX and load loops were optimized for each humidity level in order to achieve an optimal Q - factor and maximize the SCMR system's wireless efficiency. In order to re-tune the RX and load loops for each humidity level we had to increase their cross-sectional radius, $a$, as shown in Table 4-5.

Table 4-5. Humidity effects on optimized SCMR designs $(H=10 \mathrm{~cm}, D=10 \mathrm{~cm})$

\begin{tabular}{|c|c|c|c|c|}
\hline $\begin{array}{c}\text { Humidity } \\
\text { Level (\%) }\end{array}$ & $\begin{array}{c}\text { RX loop's } \\
a(\mathrm{~mm})\end{array}$ & $\begin{array}{c}\text { TX loop Q- } \\
\text { factor }\end{array}$ & $\begin{array}{c}\text { Rx loop Q- } \\
\text { factor }\end{array}$ & Efficiency (\%) \\
\hline 0.2 & 2.2 & 2650 & 2010 & 59.0 \\
\hline 2.8 & 2.4 & 2920 & 1750 & 53.5 \\
\hline 5.5 & 2.7 & 2830 & 1690 & 44.8 \\
\hline 6.2 & 2.9 & 2480 & 1640 & 42.9 \\
\hline 9.3 & 3.3 & 2250 & 1410 & 34.7 \\
\hline 12 & 3.5 & 1450 & 1320 & 27 \\
\hline
\end{tabular}




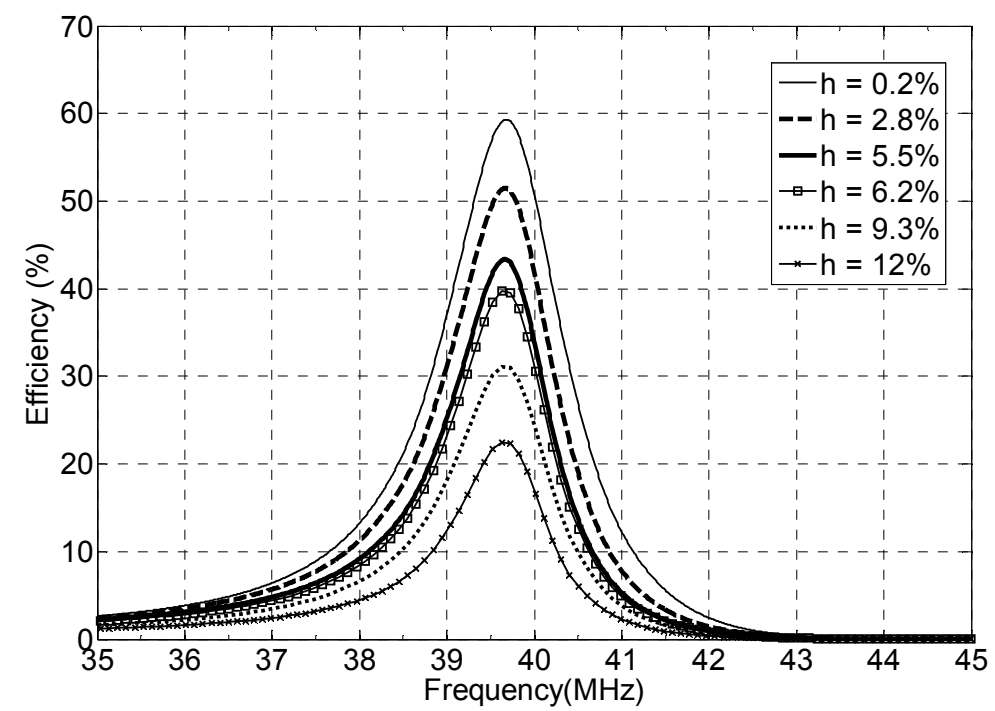

Figure 4.10: Efficiency vs. frequency in optimized SCMR designs $(D=10 \mathrm{~cm}, H=10$ $\mathrm{cm})$.

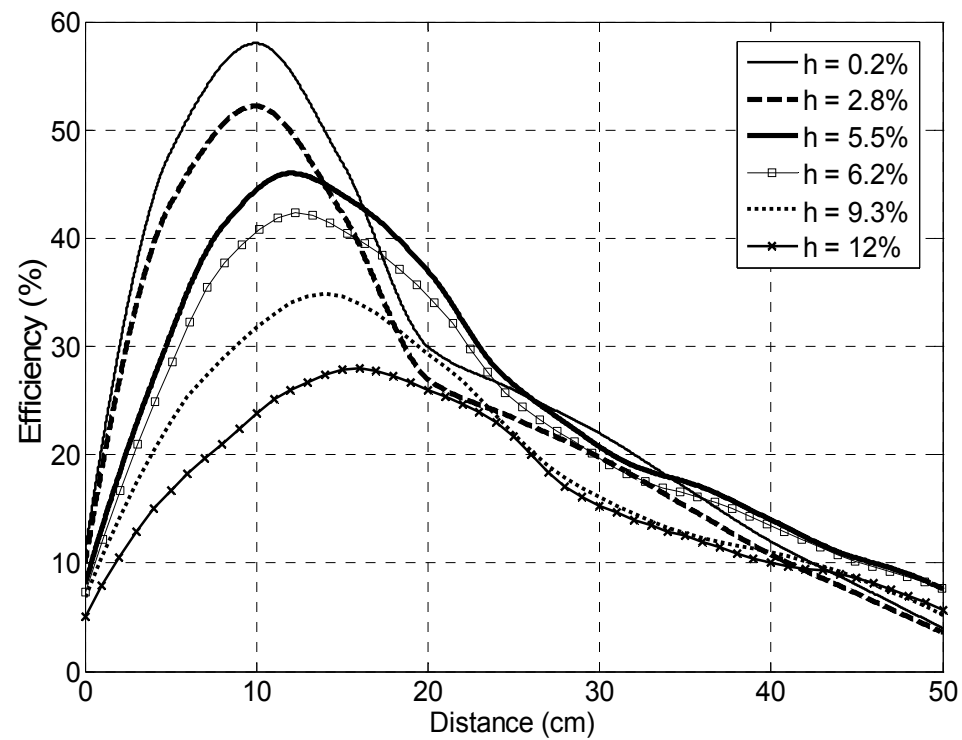

Figure 4.11: Efficiency vs. distance in optimized SCMR designs $(H=10 \mathrm{~cm})$.

The efficiency results of the optimized designs are illustrated in Table 4-5. It can be concluded from Tables 4-4 and 4-5 that by optimizing the SCMR system for each humidity, we were able to achieve significantly larger efficiencies. Therefore, depending on the application the humidity of the concrete needs to be taken into consideration when 
designing SCMR systems. Also, Figure 4.10 shows the simulated efficiency versus the frequency at $H=10 \mathrm{~cm}$ and $D=10 \mathrm{~cm}$ for different humidity levels. Finally, Figure 4.11 illustrates the simulated efficiency versus the placement depth, $D$, of RX loop for different humidity levels.

\subsubsection{Effects of Reinforced Bars on Wireless Power Transfer}

In this section, the performance of SCMR systems in reinforced concrete (i.e., concrete with rebars) is examined. Both parallel and cross rebar configurations are considered. Specifically, the SCMR system of Section 4.2 is used here with the same geometrical parameters $\left(\ell_{1}=4 \mathrm{~cm}, \ell_{3}=2 \mathrm{~cm}, H=10 \mathrm{~cm}\right)$ and operational frequency (39.37 MHz). In addition, dry concrete is assumed in this analysis (i.e. $h=0.2 \%$ ). The performance of SCMR is examined for concrete with parallel rebars (see Figure 4.12) and with cross-bars (see Figure 4.13). The rebars have a cross-sectional radius of $2.2 \mathrm{~mm}$. The spacing between the rebars, $G$, is $10 \mathrm{~cm}$. The distance, $g$, between the SCMR receiver and the rebars is $3 \mathrm{~cm}$. The set depth of the rebars inside concrete, $t$, is fixed to $10 \mathrm{~cm}$. In our analysis, the embedded depth of the SCMR receiver, $D$, is varied between $10 \mathrm{~cm}$ and $50 \mathrm{~cm}$. The results are illustrated in Table 4-6 and they show that SCMR can be used to efficiently transfer power through reinforced concrete. It can be also concluded, that SCMR exhibits the largest efficiency for plain concrete and the smallest efficiency for reinforced concrete with crossbars. 


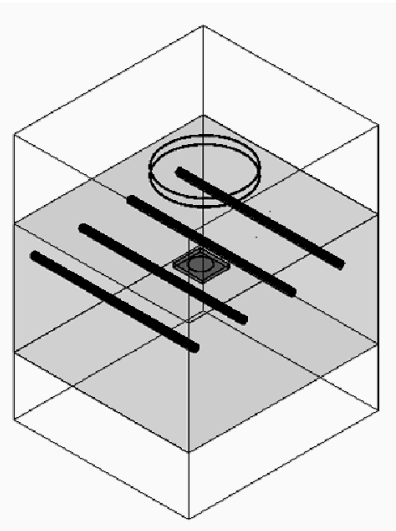

(a)

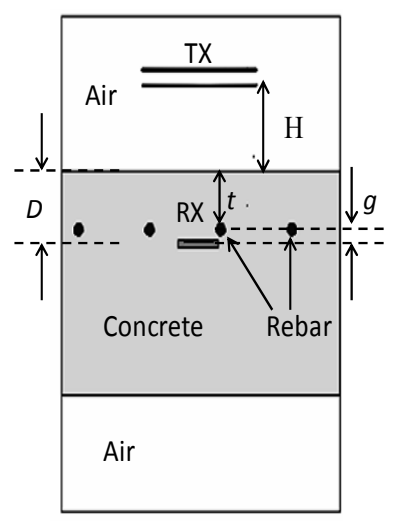

(b)

Figure 4.12: An SCMR system inside concrete with parallel rebars. (a) 3D view. (b) 2D view.

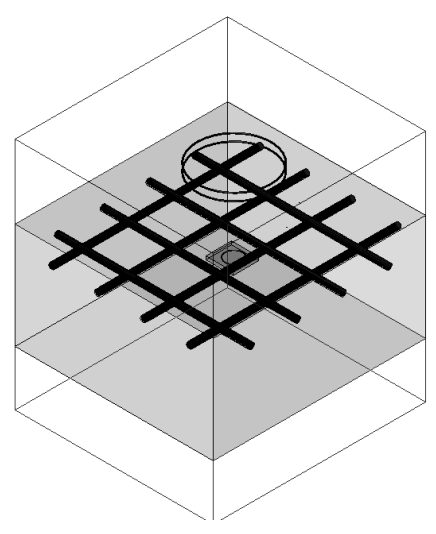

(a)

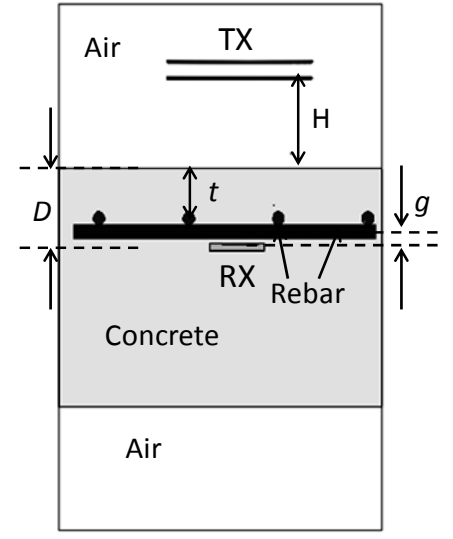

(b)

Figure 4.13: An SCMR system inside concrete with parallel crossbars. (a) 3D view. (b) $2 \mathrm{D}$ view.

Furthermore, the efficiencies observed in all three cases of Table 4-6 are significantly larger than the efficiencies achieved by traditional inductive coupling techniques [96].

Table 4-6. Comparison of Efficiencies for varying RX depth $\left(\ell_{1}=4 \mathrm{~cm}, \ell_{3}=2 \mathrm{~cm}\right)$

\begin{tabular}{|c||c|c|c|}
\hline \multirow{2}{*}{$D(\mathrm{~cm})$} & \multicolumn{3}{|c|}{ Efficiency (\%) } \\
\cline { 2 - 4 } & Plain Concrete & $\begin{array}{c}\text { Concrete with parallel } \\
\text { rebars }\end{array}$ & $\begin{array}{c}\text { Concrete with } \\
\text { crossbar rebars }\end{array}$ \\
\hline 10 & 59 & 53 & 39 \\
\hline 20 & 48 & 46 & 35 \\
\hline 30 & 35 & 32 & 25 \\
\hline 40 & 29 & 23 & 15 \\
\hline 50 & 14 & 10 & 5 \\
\hline
\end{tabular}




\subsubsection{Power Rectification}

In order to charge the batteries of sensors that are embedded in concrete, DC power is required. Therefore, the RF power that is wirelessly transferred to the load loop needs to be rectified. Figure 4.14 shows the SCMR system integrated with a rectifying circuit. Here, the depth is $\mathrm{D}=10 \mathrm{~cm}$, and the height is $\mathrm{H}=10 \mathrm{~cm}$. The power supplied by the source is $1 \mathrm{~W}$ at $39.75 \mathrm{MHz}$. The rectifying circuit consists of one $1 \mathrm{~N} 5819$ diode and a $0.8 \mu \mathrm{F}$ capacitor. The load of the rectifier is $50 \mathrm{ohms}$.

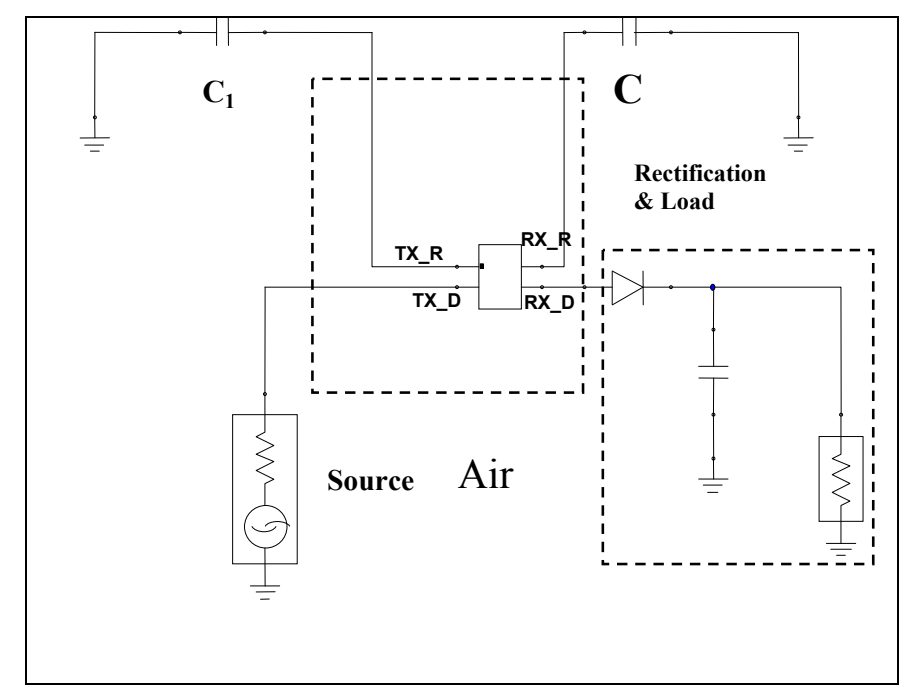

Figure 4.14: Wireless power transfer with rectification.

Table 4-7. Comparison of efficiencies $(H=10 \mathrm{~cm}, D=10 \mathrm{~cm})$

\begin{tabular}{|c|c|c|}
\hline $\begin{array}{c}\text { Humidity level } \\
(\%)\end{array}$ & $\begin{array}{c}\text { Efficiency before } \\
\text { rectification (\%) }\end{array}$ & $\begin{array}{c}\text { Efficiency } \\
\text { after rectification (\%) }\end{array}$ \\
\hline 0.2 & 59.0 & 38.5 \\
\hline 2.8 & 53.5 & 35.4 \\
\hline 5.5 & 44.8 & 29.3 \\
\hline 6.2 & 42.9 & 27.8 \\
\hline 9.3 & 34.7 & 21.7 \\
\hline 12 & 27 & 17.2 \\
\hline
\end{tabular}


The analysis of the power rectification was carried out using Ansoft Nexxim. Our maximum rectified power was recorded at $0.2 \%$ humidity (dry concrete) with a rectification efficiency of $65.4 \%$. The maximum overall efficiency of this SCMR system was $38.5 \%$ after rectification (compared to an efficiency of $59.0 \%$ before the rectification). A comparison of the efficiencies before and after rectification is shown in Table 4-7 for different humidity levels.

\subsection{Wireless Power Transfer with Helix Structures}

Wireless power transfer across two different media (air-to-concrete) is significantly different from homogenous interface. The RX helix design described in the previous section (air-to-air power transfer) must be changed for the air-to-concrete power transfer. This is because the electrical properties of a helix depend on the material properties of the medium where it is embedded. When the RX helix designed above was embedded in dry concrete $(0.2 \%$ humidity in simulation and dry concrete in measurements) without any other changes, we observed that the efficiency of the air-to-air system at $\ell_{2}=15 \mathrm{~cm}$ reduced significantly from $81.3 \%$ to approximately $25 \%$. This problem is addressed by re-designing the RX helix so that the sensor embedded in concrete (see Figure 4.15) is resonant and exhibits high $\mathrm{Q}$ factor at $f_{r}$. The design of the TX helix remained the same $\left(r=6 \mathrm{~cm}, r_{c}=0.22 \mathrm{~cm}, N=9\right)$ since it is still located in air as shown in Figure 4.15. The RX helix embedded in concrete redesigned yielding the following geometrical parameters: $r=4.9 \mathrm{~cm}, r_{c}=0.22 \mathrm{~cm}$, and $N=11$. The air-box was added because we observed through simulations and experimentations that when the RX helix and load loop were inside the air box, instead of touching the concrete, the Q factor 
as well as the power transfer efficiency improve significantly. In addition, similar configuration to our air box was used by [70], where an air-gap was added on top of patch antennas embedded in concrete in order to optimize their performance. Practically, the air box around the RX helix and load loops can be implemented by using appropriate packaging.

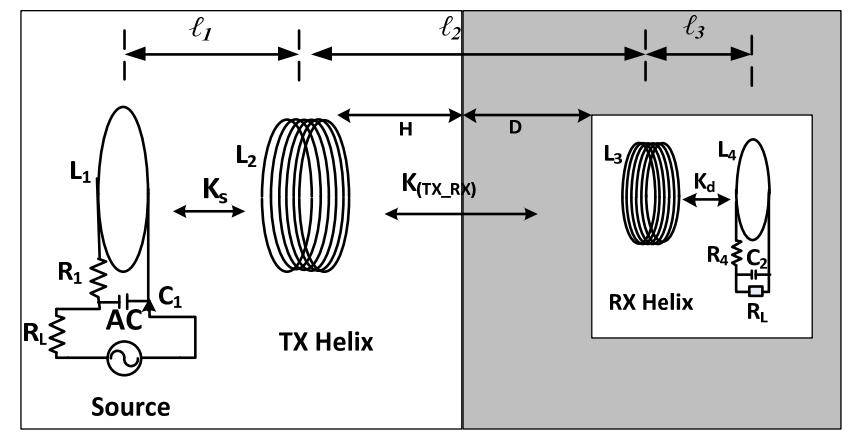

Figure 4.15: SCMR in non-homogenous interface with helices.

Also, the distances of this design (see Figure 4.15) are as follows: $\ell_{1}=2 \mathrm{~cm}, \ell_{2}=$ $H+D, \ell_{3}=2 \mathrm{~cm}$. Figure 4.16 shows two different views of the measurement setup. Figure 4.16 (a) shows the top view of the setup, exposing the dry concrete embedded sensor, while Figure 4.16 (b) shows the side view of the entire setup. A vector network analyzer was used for all measurements. Figure 4 shows the simulation and measurement results for concrete of $0.2 \%$ humidity and for a depth, $D=10 \mathrm{~cm}$ and for two different heights, $H$, (see Figure 4.15). It is observed that this design achieved a wireless power transfer efficiency of $62.6 \%$ shows the maximum wireless power transfer efficiency occurs at our desired frequency, $f_{r}$ of $27.2 \mathrm{MHz}$. The simulation results closely agree with the measurements, and the discrepancies can be attributed to the non perfect geometrical uniformity of the helical coils (versus the perfect simulated coils) which we built in our 
laboratory. Based on both our simulated and measured results, this SCMR wireless power system for sensors embedded in concrete achieved efficiencies that are significantly larger than the efficiencies achieved by typical RFID [69], [3] systems using conventional resonant loops. Tables 4-8 and 4-9 shows the S-parameters and efficiency at $h=2.2 \%$ and $5.5 \%$.

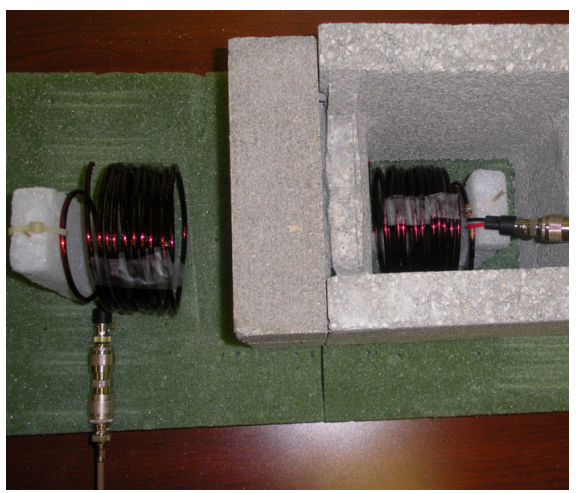

(a)

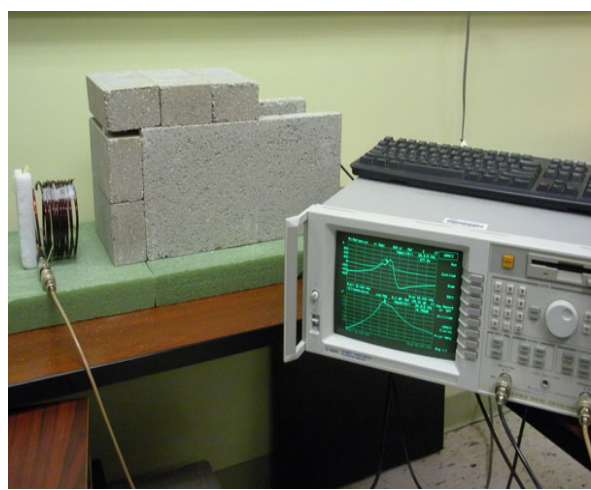

(b)

Figure 4.16: Two different views of the measurement setup. (a) Top view with the top concrete slabs removed. (b) Side view of the setup.

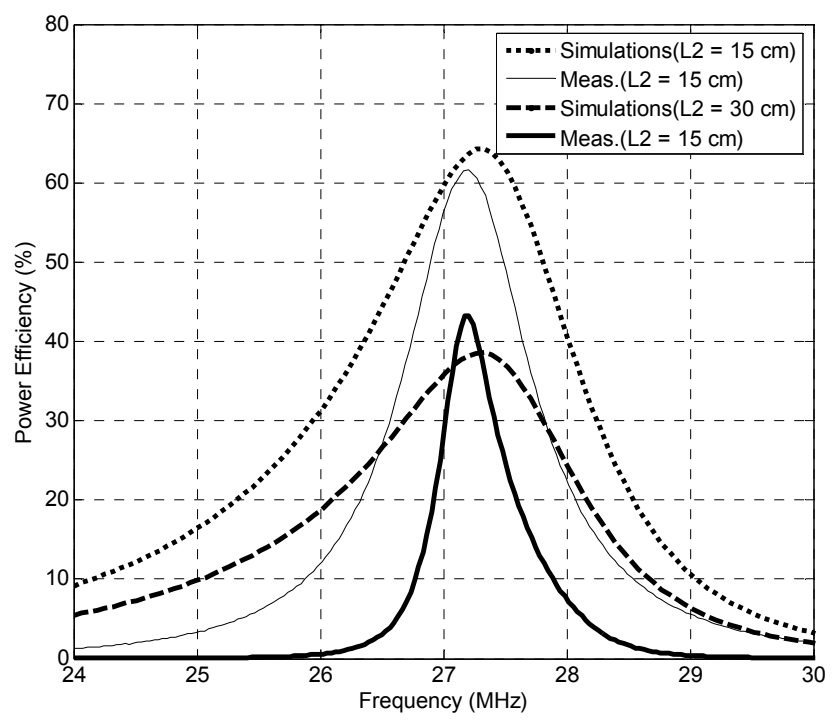

Figure 4.17: Efficiency of simulation and measurement results for $27.2 \mathrm{MHz}(h=0.2 \%)$. 
Table 4-8. Efficiency at humidity level, $h=2.2 \%$

\begin{tabular}{|c|c|c|c|}
\hline \multirow{2}{*}{$\begin{array}{c}\text { Depth, D } \\
(\mathrm{cm})\end{array}$} & \multicolumn{3}{|c|}{ S parameters and Efficiency } \\
\cline { 2 - 4 } & $S_{11}(d B)$ & $S_{12}(d B)$ & Efficiency $(\%)$ \\
\hline 5 & -1.7 & -7.45 & 56.0 \\
\hline 10 & -1.9 & -8.30 & 41.2 \\
\hline 15 & -2.1 & -9.60 & 33.4 \\
\hline
\end{tabular}

Table 4-9. Efficiency at humidity level, $h=5.5 \%$

\begin{tabular}{|c|c|c|c|}
\hline \multirow{2}{*}{$\begin{array}{c}\text { Depth, D } \\
(\mathrm{cm})\end{array}$} & \multicolumn{3}{|c|}{ S parameters and Efficiency } \\
\cline { 2 - 4 } & $S_{11}(d B)$ & $S_{12}(d B)$ & Efficiency $(\%)$ \\
\hline 5 & -1.95 & -8.10 & 44.6 \\
\hline 10 & -2.19 & -9.15 & 31.1 \\
\hline 15 & -2.30 & -10.10 & 26.2 \\
\hline
\end{tabular}

\subsection{Wireless Power Transfer with Spiral}

\subsubsection{WPT in Plain Concrete}

The setup for the air-concrete interface with spiral resonators is shown in Figure 4.18. In order to achieve high efficiency, $\mathrm{TX}$ and $\mathrm{RX}$ spiral must be designed to resonate at the desired operational frequency and coincide with the frequency both naturally exhibit maximum Q-factor.

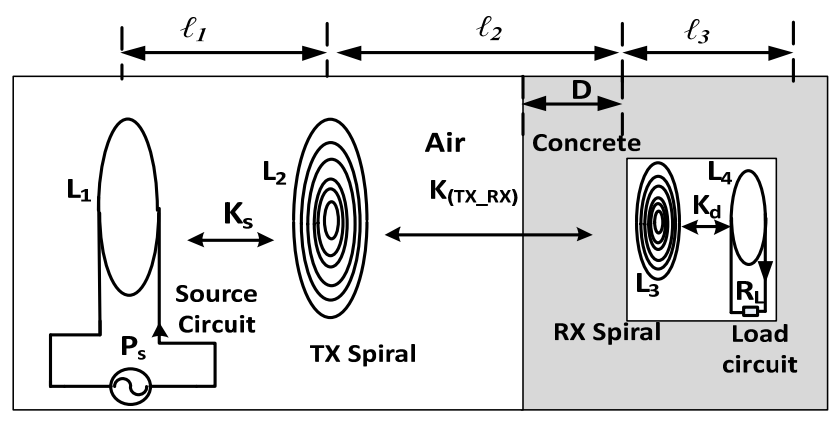

Figure 4.18: Schematic of SCMR system with spirals. 
The spirals are made from $0.5 \mathrm{~mm}$ copper sheet, the embedded and external spirals are $2.5 \mathrm{~cm}$ and $8 \mathrm{~cm}$ diameter respectively. The SCMR system of Figure 4.18 was simulated in Ansoft HFSS, at three different concrete depth, $D$, and humidity levels, $h$ at $40.65 \mathrm{MHz}$ ISM band.

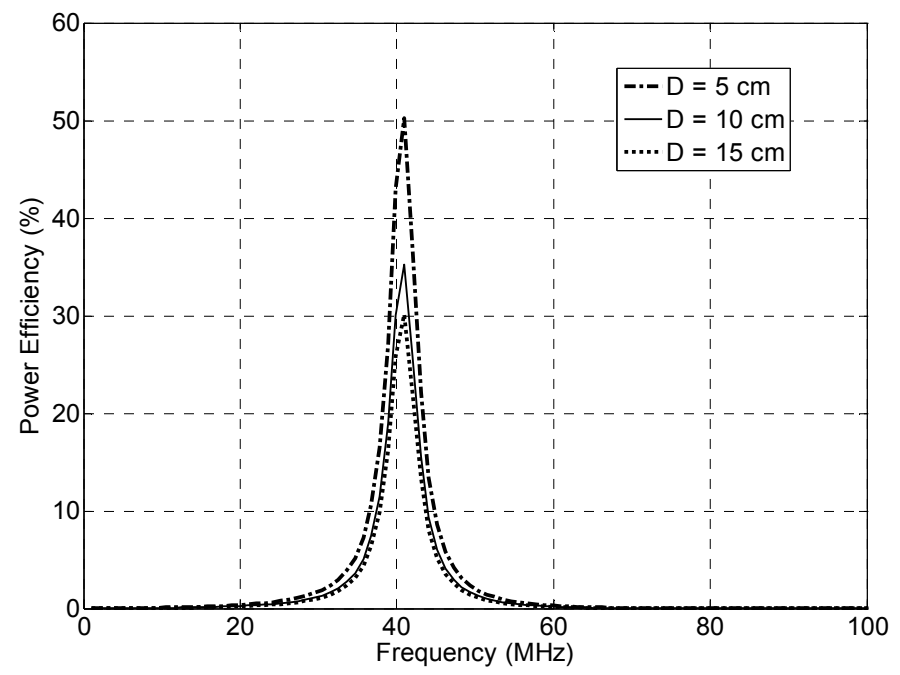

Figure 4.19: Efficiency at humidity level, $h=0.2 \%$.

Figure. 4.19 shows the efficiencies achieved at $h=0.2 \%$ for three different placement depth. The Tables 4.10 show the S-parameters and the efficiencies achieved at $h=2.2 \%$. These results show that spiral resonant element can achieve efficient WPT.

Table 4-10. Efficiency at humidity level, $h=2.2 \%$

\begin{tabular}{|c|c|c|c|}
\hline \multirow{2}{*}{$\begin{array}{c}\text { Depth, D } \\
(\mathrm{cm})\end{array}$} & \multicolumn{3}{|c|}{ S parameters and Efficiency } \\
\cline { 2 - 4 } & $S_{11}(d B)$ & $S_{12}(d B)$ & Efficiency (\%) \\
\hline 5 & -2.15 & -7.00 & 51.40 \\
\hline 10 & -2.30 & -8.50 & 34.30 \\
\hline 15 & -2.70 & -9.60 & 25.10 \\
\hline
\end{tabular}




\subsubsection{WPT in Reinforced Concrete}

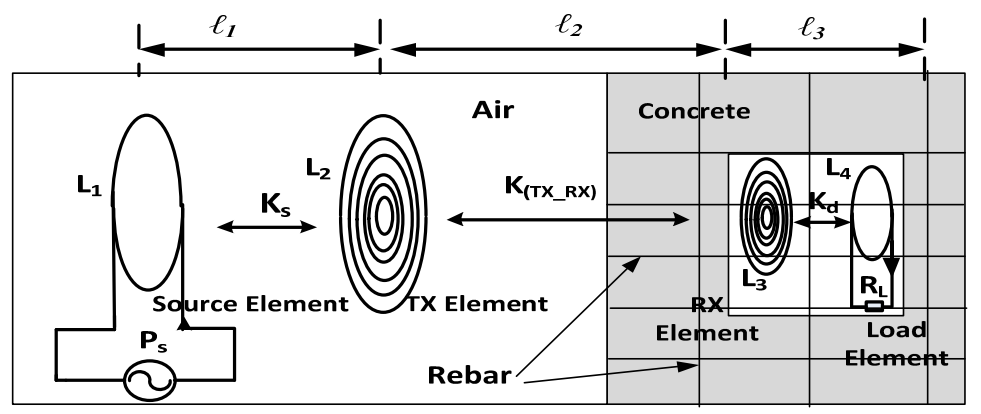

Figure 4.20: Schematic of the SCMR power transfer system with rebar.

The SCMR system in Figure. 4.20 was simulated in Ansoft HFSS for different rebar configurations at depth, $\mathrm{D}=10 \mathrm{~cm}$, and for various humidity levels, $h$, at $40.65 \mathrm{MHz}$ ISM band. The spirals are made from $0.5 \mathrm{~mm}$ copper sheet, the embedded and external spirals are $2.5 \mathrm{~cm}$ and $8.2 \mathrm{~cm}$ diameter, respectively.

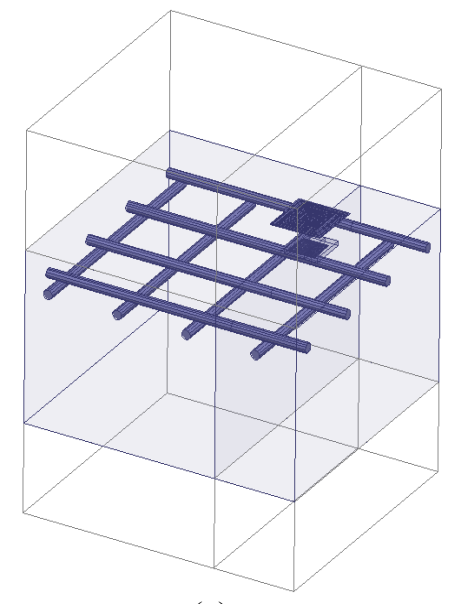

(a)

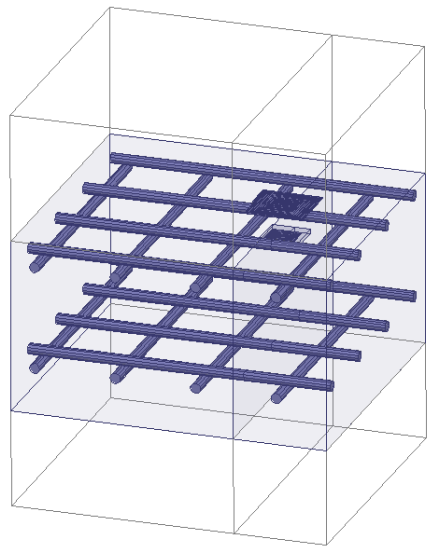

(b)

Figure 4.21: Different rebar configurrations (a) rebars on top (b) rebars on top and bottom.

Figure 4.21 shows two different rebar configurations for the reinforced concrete. The Table 4-12 and Table 4-13 show the S-parameters and the efficiencies achieved at humidity level, $h=2.2 \%$ for different placement $D$ for the configuration of Figure 4.21(a). 


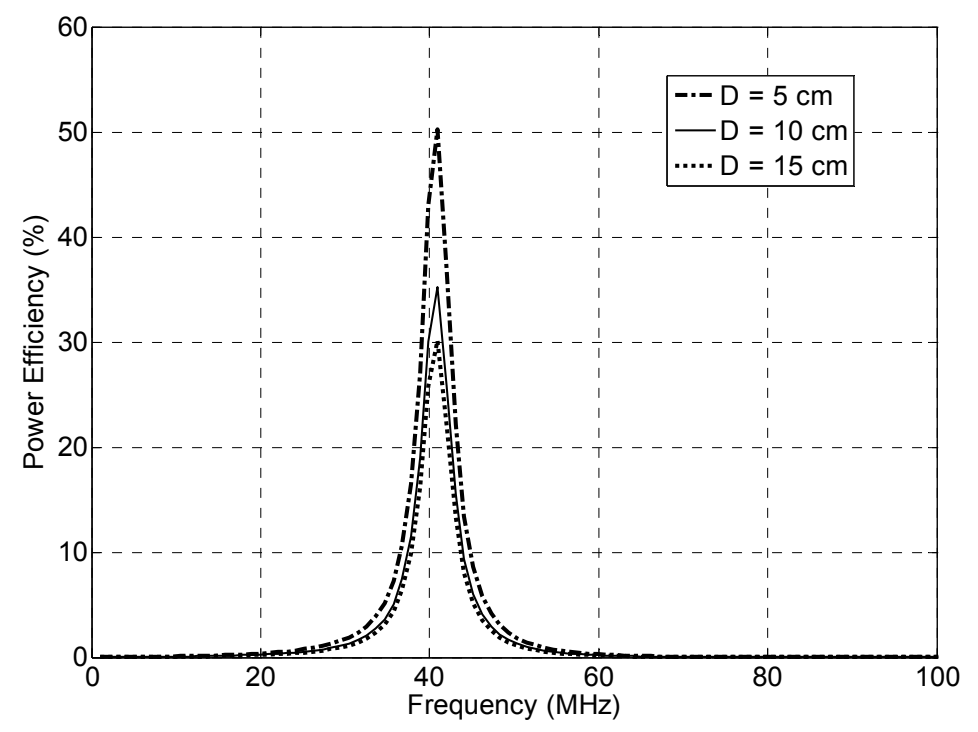

Figure 4.22: Efficiency at humidity levels, $h=0.2 \%$.

Table 4-11. Efficiency at humidity level, $h=2.2 \%$

\begin{tabular}{|c|c|c|c|}
\hline \multirow{2}{*}{$\begin{array}{c}\text { Depth, D } \\
(\mathrm{cm})\end{array}$} & \multicolumn{3}{|c|}{ S parameters $(\mathrm{dB})$ and Efficiency $(\%)$} \\
\cline { 2 - 4 } & $S_{11}(d B)$ & $S_{12}(d B)$ & Efficiency $(\%)$ \\
\hline 5 & -1.90 & -6.95 & 55.40 \\
\hline 10 & -1.80 & -8.90 & 37.54 \\
\hline 15 & -1.60 & -11.40 & 29.20 \\
\hline
\end{tabular}

These results show that the spiral resonant element can be used to efficiently deliver power to sensors deep in reinforced concrete, even with rebars.

\subsection{Summary}

An efficient SCMR wireless power transfer system was developed for powering sensors embedded in both plain and reinforced concrete using the SCMR method. SCMR systems based on loops achieved maximum efficiencies that range from $27 \%$ at $12 \%$ humidity to $59.0 \%$ at $0.2 \%$ humidity inside concrete at a depth of $10 \mathrm{~cm}$ before 
rectification. After rectification the maximum efficiency ranged from $17.2 \%$ at $12 \%$ humidity to $38.5 \%$ at $0.2 \%$ humidity. The SCMR system with helical elements achieved efficiencies in dry concrete at $27.2 \mathrm{MHz}$ that range from $62.6 \%$ at $15 \mathrm{~cm}$ depth to $38.3 \%$ at $30 \mathrm{~cm}$ depth, and the spirals achieved efficiencies that range from $56.0 \%$ at $5 \mathrm{~cm}$ depth to $25.1 \%$ at $15 \mathrm{~cm}$ depth. The efficiencies reported here for the SCMR method are significantly larger than typical efficiencies achieved by conventional wireless powering methods. Therefore, the SCMR method can be used to efficiently transfer power wirelessly to embedded sensors in concrete structures. Finally, based on our results, SCMR is expected to be highly efficient for wireless power transmission through other non-homogeneous media interfaces. 


\section{CHAPTER 5}

\section{WIRELESS POWERING OF WEARABLE AND IMPLANTABLE MEDICAL DEVICES}

In this chapter, we study the WPT of Wearable and Implantable Medical Devices (WIMD). Computational methods are applied to calculate the efficiency of the different WPT systems. The Specific Absorption Rate (SAR) calculations are also performed.

\subsection{Powering of Wearable and Implantable Medical Devices}

When developing wireless power transmission system for Implantable Medical Devices (IMDs), the power lever is restricted. Too little power might lead to system malfunction, and excessive power might cause overheating of the tissue and may result in significant tissue damage [77]. This restriction requires the design of low power source with high transmission efficiency for such system [100]. The Federal Communications Commission (FCC) specifies that the exposure to radio frequency (RF) energy from wireless devices must be limited to a maximum SAR value of $1.6 \mathrm{~W} / \mathrm{kg}$ for $1 \mathrm{~g}$ of tissue, beyond which significant tissue heating and damage may occur. In this chapter, we simulate WPT system near or inside a human phantom model in HFSS. The aim is to evaluate SCMR WPT system for implantable and wearable devices in terms of efficiency and safety. Our analysis will examine if SCMR systems can operate within the safety limits established by FCC [117]. 


\subsection{Wireless Power Transfer in Air-Tissue Interface via SCMR}

SCMR systems use resonant transmitters (TX) and receivers (RX) that are strongly coupled, due to their ability to exhibit maximum Q-factor and resonance at the same frequency, $f_{r}$. The setup for the air-tissue SCMR system is shown in Figure 5.1. The source loop is linked to the power source, $P_{s}$, by the coupling coefficient $k_{s}$, and on the receiver side the load loop is linked to the load, $R_{L}$, similarly with the coupling coefficient $k_{d}$.

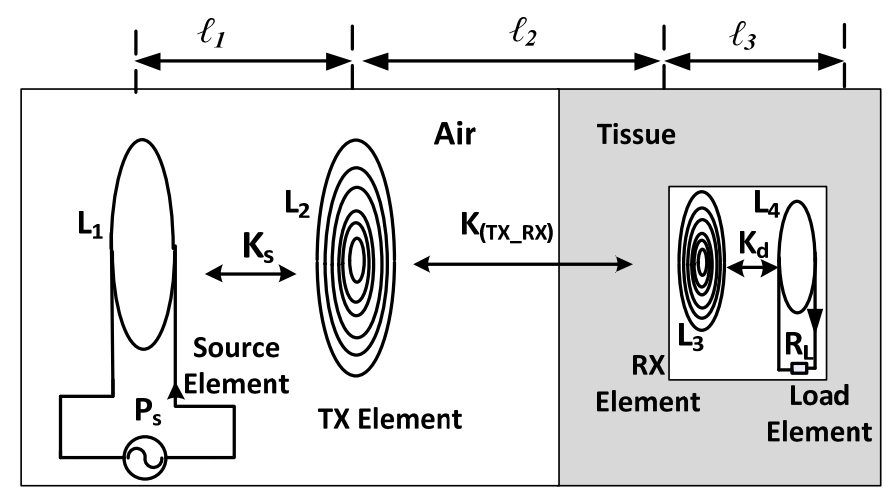

Figure 5.1: Schematic of SCMR power transfer system for tissue.

The TX and RX resonating spirals are linked by the coupling coefficient $k_{\left(T X_{-} R X\right)}$. In order to achieve high efficiency, the TX and RX spirals must be designed to resonate at the desired operating frequency that coincides with the frequency where they naturally exhibit maximum Q-factor. In the next section, novel SCMR systems for implantable and wearable devices are presented. 


\subsection{Wireless Powering with Standard SCMR}

\subsubsection{Wireless Power Transfer}

Wireless power transfer efficiencies of SCMR device are strongly constrained by the media in which they are embedded in. Table 5-1 shows the conductivities of different tissue types at different frequency bands [118]. In this work, we set up the SCMR system in the 40.65 ISM band. From Table 5-1, it can be seen that the lower frequency bands will result in less losses because of their lower conductivities.

Table 5-1. Conductivities (Siemens/m) of different tissue at various frequencies

\begin{tabular}{|c|c|c|c|c|c|}
\hline \multirow{2}{*}{ Tissue/Freq } & \multicolumn{5}{|c|}{ Conductivities at different tissues at different frequencies } \\
\cline { 2 - 6 } & $133 \mathrm{KHz}$ & $6.78 \mathrm{MHz}$ & $13.56 \mathrm{MHz}$ & $27.0 \mathrm{MHz}$ & $40.0 \mathrm{MHz}$ \\
\hline Skin & 0.0854 & 0.1471 & 0.2380 & 0.4275 & 0.4540 \\
\hline Fat & 0.0245 & 0.0278 & 0.0304 & 0.0329 & 0.0341 \\
\hline Muscle & 0.3689 & 0.6021 & 0.6282 & 0.6540 & 0.6692 \\
\hline Lung & 0.2761 & 0.4211 & 0.4516 & 0.4843 & 0.5046 \\
\hline Bone & 0.0842 & 0.1159 & 0.1285 & 0.1419 & 0.1500 \\
\hline Heart & 0.2241 & 0.4713 & 0.5262 & 0.5877 & 0.6269 \\
\hline
\end{tabular}

The simulations were carried out with HFSS and a human phantom model in which the various tissue types were characterized. Figure 5.2 shows two views of the human phantom in the presence of the standard SCMR system using rectangular spiral elements. 


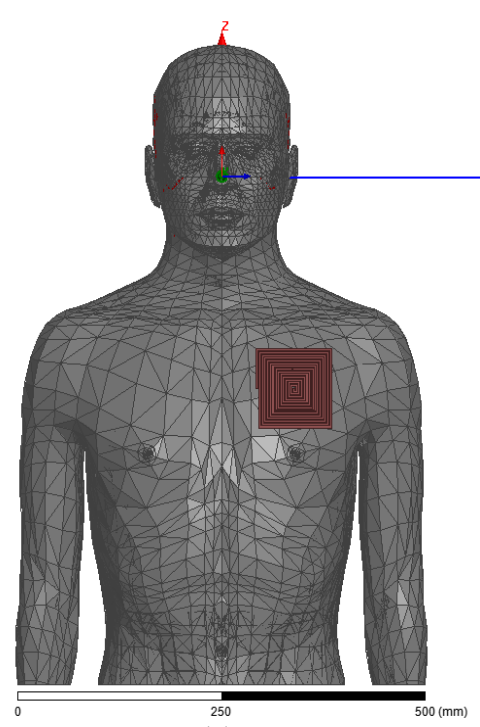

(a)

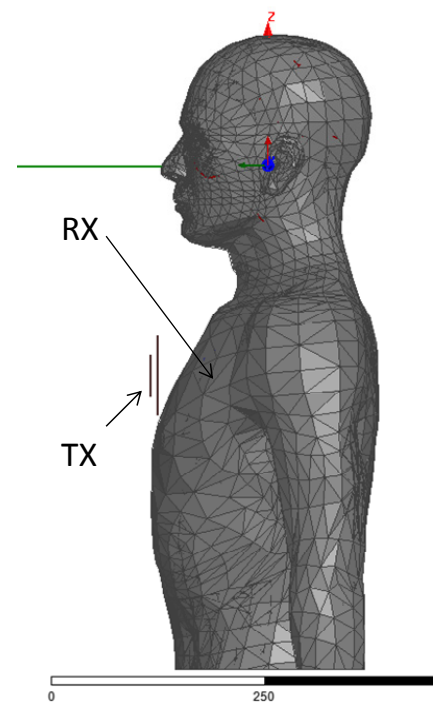

(b)

Figure 5.2: The SCMR system in tissue. (a) Front view of the SCMR system with implant. (b) Side view of the SCMR system with implants.

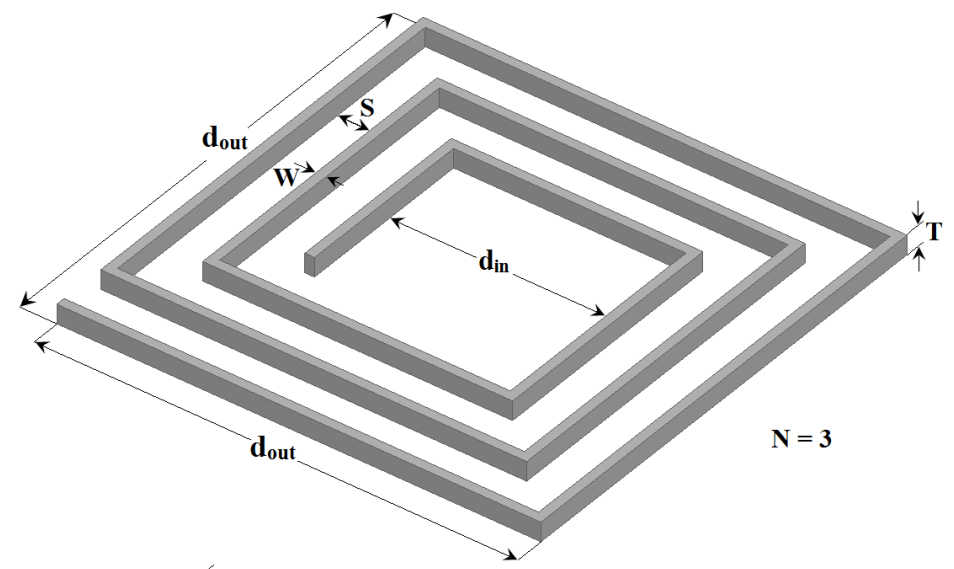

Figure 5.3: The spiral model geometry

The spiral model and specification are as shown in Figure 5.3, the dimension of the TX spiral (outside) are $d_{\text {in }}=4 \mathrm{~mm}, d_{\text {out }}=92 \mathrm{~mm}, w=2 \mathrm{~mm}, s=1 \mathrm{~mm}, N=16, \rho=$ $5.7 \times 107$ (copper), $L=11.2 u H, R_{S}=R_{L}=50$ ohms. The dimension of the $\mathrm{RX}$ (embedded) spiral model dimensions are $d_{\text {in }}=2 \mathrm{~mm} d_{\text {out }}=34 \mathrm{~mm}, \mathrm{~W}=1 \mathrm{~mm}, s=0.3$ mm, $N=16, \rho=5.7 \times 107$ (copper), $L=0.39 u H, R_{S}=R_{L}=50$ ohms. The distance between the TX and RX spiral resonator is $80 \mathrm{~mm}$. This SCMR system is designed to 
operate at $40.65 \mathrm{MHz}$, this frequency belongs to the ISM band and it was selected because is appropriate for biomedical applications. The RX resonating spiral and load loop are enclosed in a small air box to avoid direct contact with tissues, which might result in high losses. The result of the power transfer in the muscle tissue at three different depth is as shown in Figure 5.3.

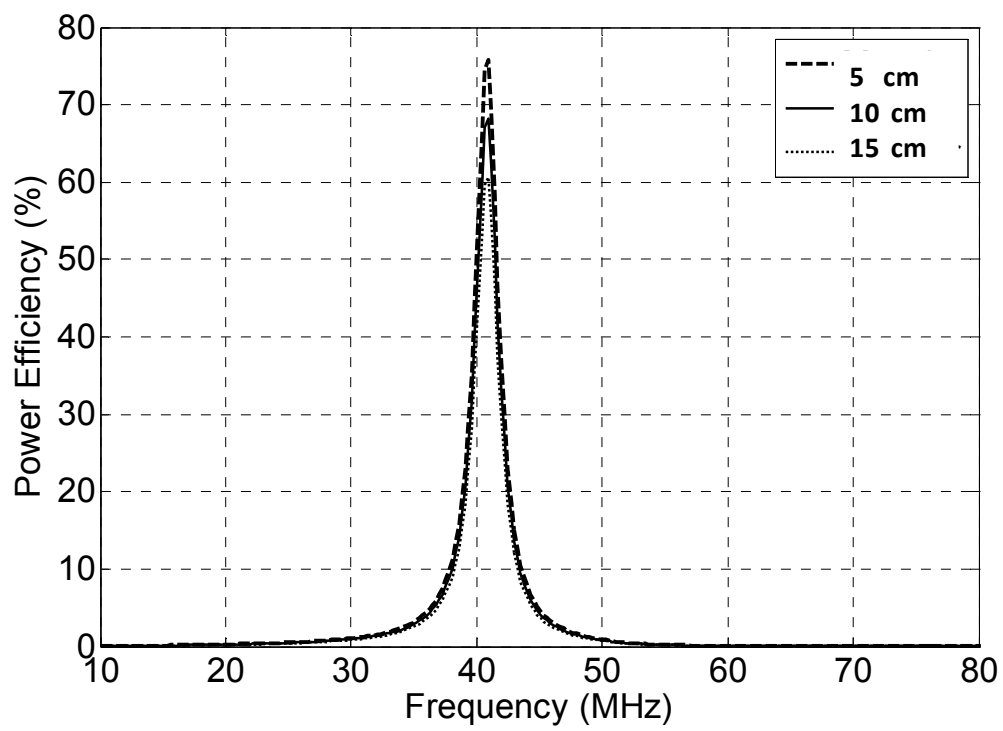

Figure 5.4: The Efficiency plots of the three different tissue types.

It is important to note that the geometrical size of the embedded RX spiral devices is still large for IMDs and more work is required to miniaturize the RX spiral for practical IMD applications. The results from Figure 5.4 show that SCMR can be used to efficiently transfer power to various human tissue in the ISM frequency band, to charge and power a wide range of power thirsty implantable devices.

\subsubsection{Specific Absorption Rate (SAR) Analysis}

The electromagnetic fields generated by the SCMR coupling causes power dissipation in the surrounding tissues, which may cause tissue heating. SAR is closely 
dependent on the electric field [119], as it can be observed from SAR equations. The simulations were carried out with Ansoft HFSS. Figure 5.5 show the distribution SAR of the human phantom.

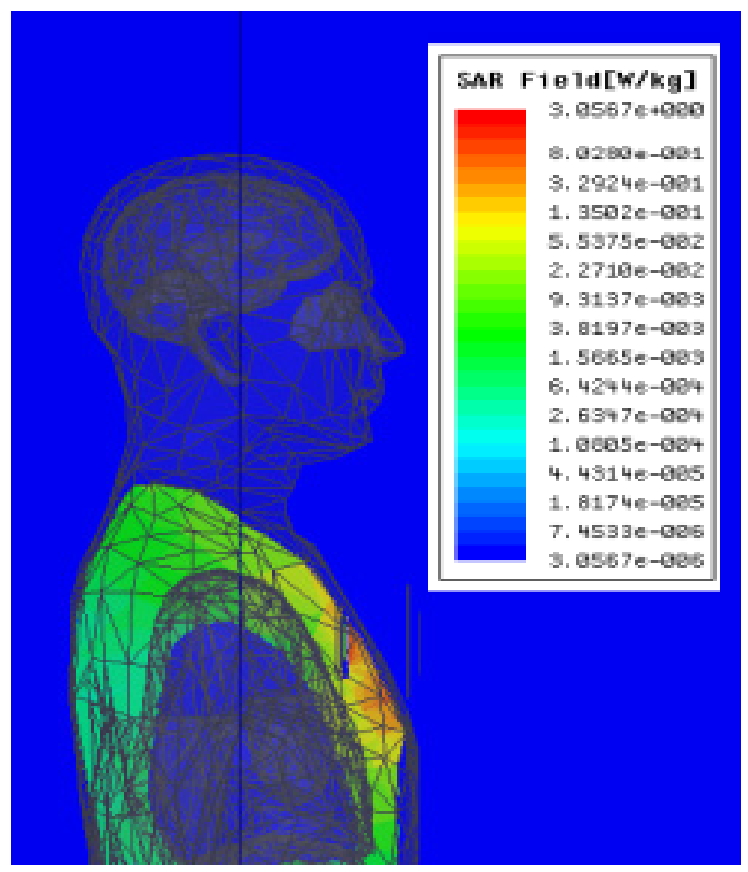

Figure 5.5: The SAR distribution for SCMR system with implants.

Table 5-2 shows the maximum electric and magnetic field, maximum SAR and the maximum efficiency, for $\mathrm{D}=5,10$ and $15 \mathrm{~cm}$. The tissue investigated is muscle tissue. This WPT can be used in a wide range of IMDs applications, such as, pacemaker defibrillators and micro-prosthetics.

Table 5-2. Human Muscle and Distance $(D=5,10$ and $15 \mathrm{~cm})$

\begin{tabular}{|c|c|c|c|c|}
\hline \multirow{2}{*}{$\begin{array}{c}\text { Distance, D } \\
(\mathrm{cm})\end{array}$} & \multicolumn{4}{|c|}{ Field Parameters and Efficiency } \\
\cline { 2 - 5 } & $\begin{array}{c}\text { Max E-field } \\
(\text { V/m) }\end{array}$ & $\begin{array}{c}\text { Max M-field } \\
(\text { A/m) }\end{array}$ & $\begin{array}{c}\text { Max SAR } \\
(\text { W/kg })\end{array}$ & $\begin{array}{c}\text { Efficiency } \\
(\%)\end{array}$ \\
\hline 5 & 400 & 24 & 3.05 & 76.20 \\
\hline 10 & 100 & 9.6 & 0.75 & 62.00 \\
\hline 15 & 65 & 4.8 & 0.15 & 58.70 \\
\hline
\end{tabular}


The results of Figure 5.4 and Table 5-2 show that the spiral resonant element can be used to efficiently deliver power to sensors implanted in the human body, the SAR exceeds the FCC limits for $D=5 \mathrm{~cm}$, but it is significantly smaller than the FCC limits for $D=10 \mathrm{~cm}$ and $15 \mathrm{~cm}$. Therefore, it is very important that a safe compromise is reached between SAR requirements and efficiency when SCMR systems are used for IMDs, in order to prevent tissue heating and damage.

\subsection{Conformal Strongly Coupled Magnetic Resonance (CSCMR)}

\subsubsection{Conformal Structure for Wireless Power Transfer}

A typical SCMR system is formed by a transmitter and a receiver resonator (self resonant elements, such as, helices, spirals, etc., can be used or loops with capacitors can also be used) and two single loops acting as the load and source devices, as shown in Figure 5.6(a), [11]. In the setup of Figure 5.6(a), $\ell_{1}$ is the distance between source loop and TX resonator, $\ell_{2}$ is the distance between the TX and RX resonator and $\ell_{3}$ is the distance between the load loop and RX resonator. SCMR systems use resonant transmitters and receivers that are strongly coupled, and able to transfer energy efficiently. In order for SCMR to achieve high efficiency, the TX and RX resonators (typically loops or coils) must be designed so that they resonate at the desired operational frequency that must coincide with the frequency at which the resonators exhibit maximum Q-factor. As seen in Figure 5.6(a), a conventional SCMR system requires certain spacing $\left(\ell_{1}\right.$ and $\left.\ell_{3}\right)$ between the source and load elements and the TX and $\mathrm{RX}$ resonators, respectively. It has been shown that for each SCMR design an optimum 
distance exists where the efficiency is maximum. In fact, previous work on SCMR has showed that if distances $\ell_{1}$ and $\ell_{3}$ smaller than their optimum values are used then the efficiency decreases. These spacing requirements make TX (source and TX resonator) and RX (load and RX resonator) systems for SCMR systems three dimensional and bulky in volume. Here, a new SCMR method, which flattens traditional SCMR systems, is proposed. Figure 5.6(b) shows the proposed Conformal SCMR (CSCMR) that shrinks the source and load elements in size and places them on the same plane with the TX and RX resonators, respectively, thereby providing conformal TX and RX systems. CSCMR systems are more suitable for IMDs and wearable biosensors and devices since they can be implemented in planar circuits and flexible substrates thereby minimizing their volume and yielding conformal devices.

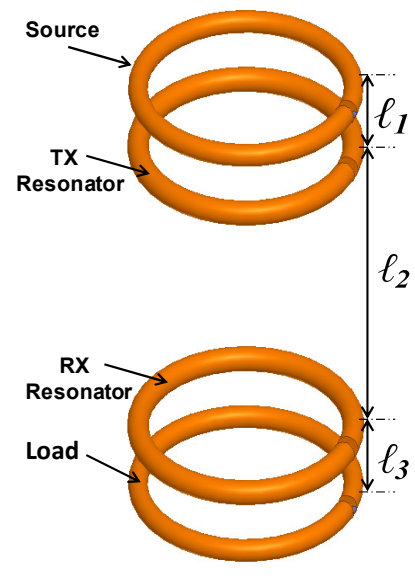

(a)

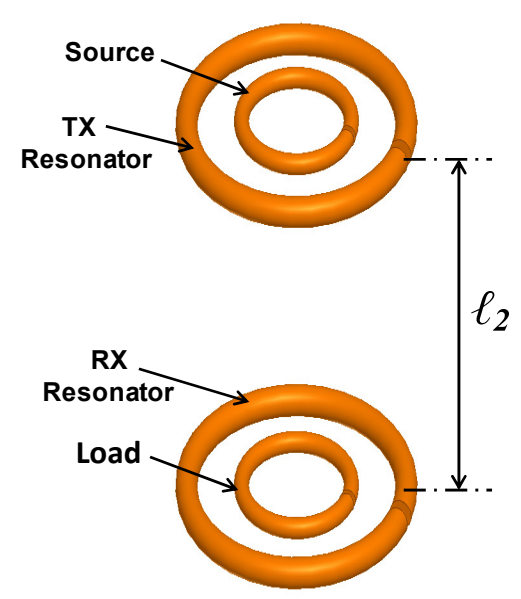

(b)

Figure 5.6: Schematic of an SCMR system in air.

In what follows, the performance of CSCMR is compared through simulations and measurements with the performance of conventional SCMR. In order to achieve maximum efficiency for both SCMR and CSCMR systems, the TX and RX resonators 
are designed to resonate at the frequency where the loops exhibit maximum Q-factor. Previously established analytical quasi-static and simulation models are used to calculate the frequency where the loops exhibit maximum Q-factor, and fine tune their design. In this case, the resonant frequency is $46.5 \mathrm{MHz}$ for the SCMR and CSCMR designs and the RX and TX loops are resonated at this frequency using a $68 \mathrm{pF}$ capacitor. HFSS is used for all simulations. The measurement setups for SCMR and CSCMR are shown in Figure 5.7. All loops in Figure 5.6(a) and 5.6(b) have a cross-sectional radius of $2.2 \mathrm{~mm}$, and conductivity of copper, $\sigma=5.8 \times 10^{7} \mathrm{~S} / \mathrm{m}$. The SCMR system of Figure 5.6(a) uses identical loops that all have a radius of $4.5 \mathrm{~cm}$. The TX and RX resonators of the CSCMR system in Figure 5.6(b) has a radius of $4.5 \mathrm{~cm}$, which is same with a radius of the loops in the conventional SCMR system. Also, the radius of CSCMR load and source loops is $2.5 \mathrm{~cm}$. The distances between the loops are as follows: $\ell_{2}=10 \mathrm{~cm}$, and $\ell_{1}=\ell_{3}=$ $1.5 \mathrm{~cm}$. Distances $\ell_{1}$ and $\ell_{3}$ were optimized using simulation to provide optimal efficiency in the SCMR system.

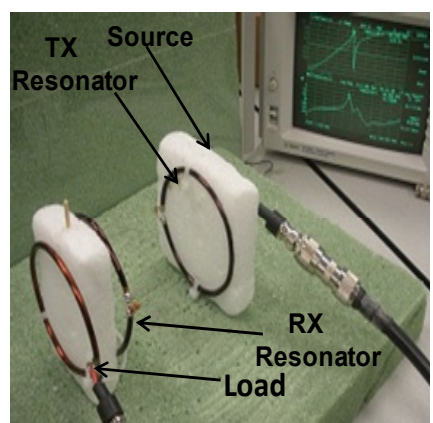

(a)

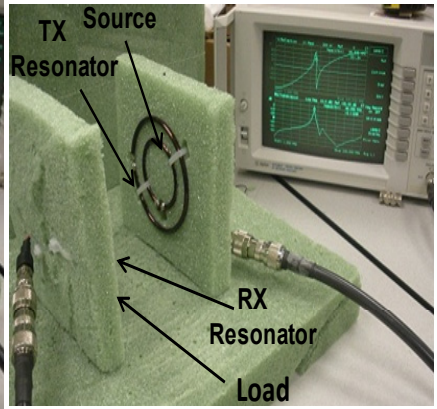

(b)

Figure 5.7: Loop-based wireless powering systems. (a) SCMR, and (b) CSCMR.

Figure 5.8 compares the $\mathrm{S}_{21}$-parameter and efficiency of the SCMR and CSCMR systems using simulations and measurements. It shows that CSCMR achieves high 
efficiency similar in magnitude to the efficiency of SCMR for the same size resonators and the same distance between the resonators. In fact, both the measurements and the simulations show that CSCMR exhibits slightly larger efficiency than SCMR. This may be partially attributed to the fact that the source and load resonators for CSCMR are embedded into the TX and RX resonators, and therefore, they are physically closer by an amount equal to distance $\ell_{1}+\ell_{3}$ (i.e., the distance between the source and load of the SCMR system is $\ell_{1}+\ell_{2}+\ell_{3}$ whereas for the CSCMR system is $\ell_{2}$ ).

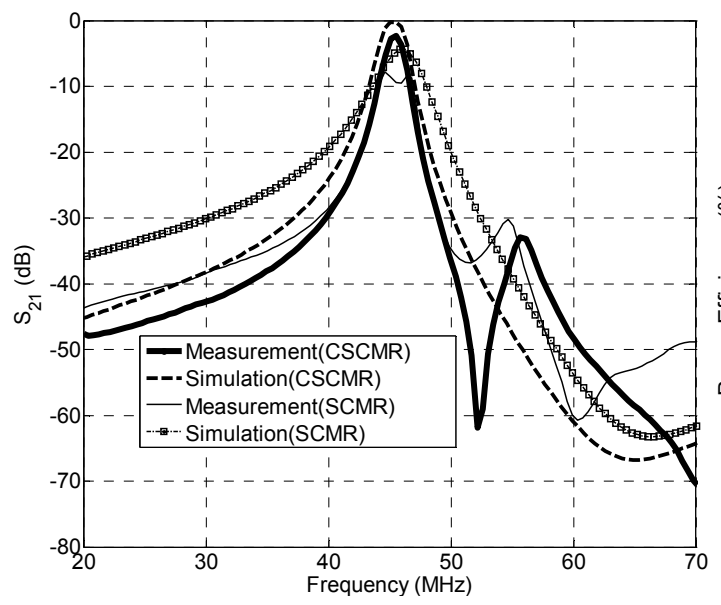

(a)

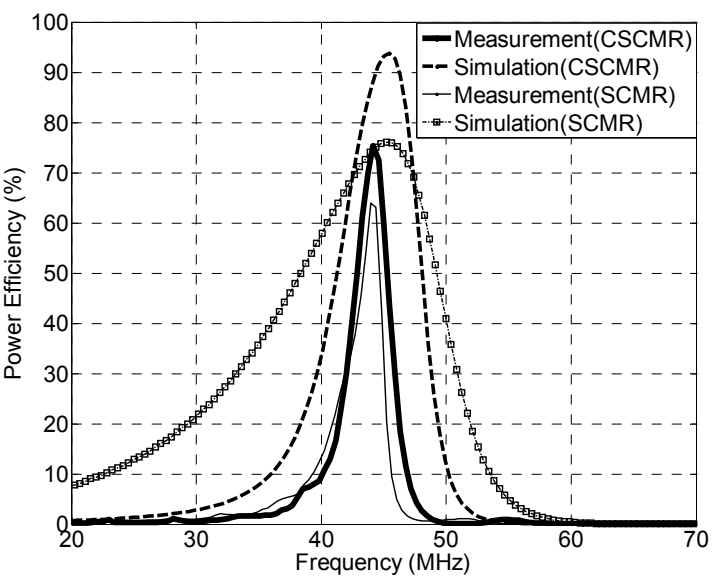

(b)

Figure 5.8: Performance comparison of SCMR and CSCMR: (a) $\mathrm{S}_{12}$ and (b) efficiency.

CSCMR is a very promising wireless powering method because it achieves large efficiencies similar to SCMR and it is conformal thereby requiring minimal volume. Therefore, CSMR is more suitable than SCMR for wireless powering of several mobile devices. The CSCMR was be extended to have multiple RX and TX resonators, with the following specifications: $\ell_{2}=10 \mathrm{~cm}$, Source loops radius $=1.5 \mathrm{~cm}, \mathrm{TX}_{1}$ and $\mathrm{RX}_{1}$ radius $=$ $2.2 \mathrm{~cm}, \mathrm{TX}_{2}$ and $\mathrm{RX}_{2}$ radius $=6.5 \mathrm{~cm}, r_{c}=2.2 \mathrm{~mm}$. The measurement model is as shwon in Figure 5.9(a) and the efficiency plot is as shown in Figure 5.9(b). 


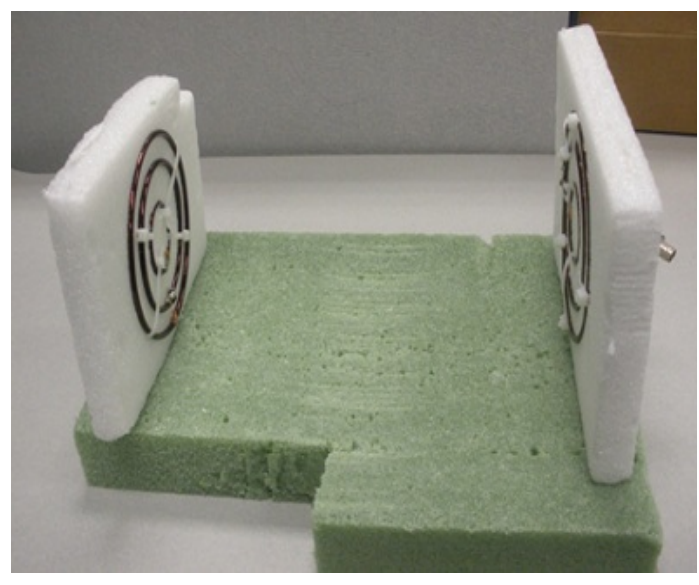

(a)

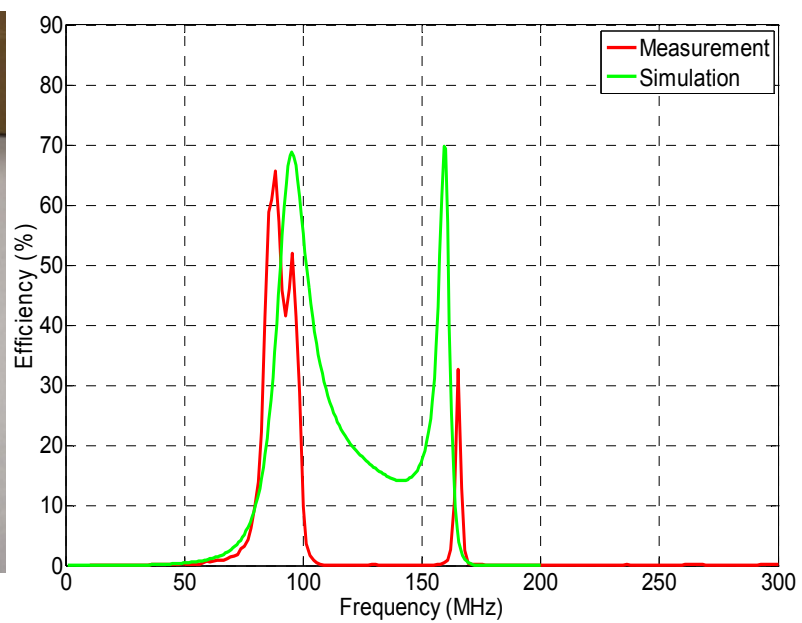

(b)

Figure 5.9: Multiband CSCMR: (a) Model (b) efficiency.

\subsubsection{Conformal Bifilar Structure for Wireless Power Transfer}

In this section, a planar novel Conformal SCMR (CSCMR) system, which is suitable for wireless powering of biomedical devices, is presented [120]. In addition, the performance of the proposed CSCMR system is compared to the performance of traditional resonant inductive coupling and SCMR. Here, all three wireless powering systems are designed based on self-resonating bifilar spirals shown in Figure 5.10. Selfresonating TX and RX elements (i.e., helices and spirals) are preferred for SCMR systems because external capacitors lower the Q-factor and in-turn the efficiency of SCMR systems. Bifilar resonators are used here because they can be excited at their center thereby facilitating a direct comparison of the performance of the three wireless powering systems shown in Figure 5.11. The basic geometrical parameters of a bifilar spiral are $N, W, S, T$ and $d_{\text {out }}$, which are the number of turns, cross-sectional width, 
spacing between turns, thickness of the trace material, and the outermost side length of the spiral, respectively.

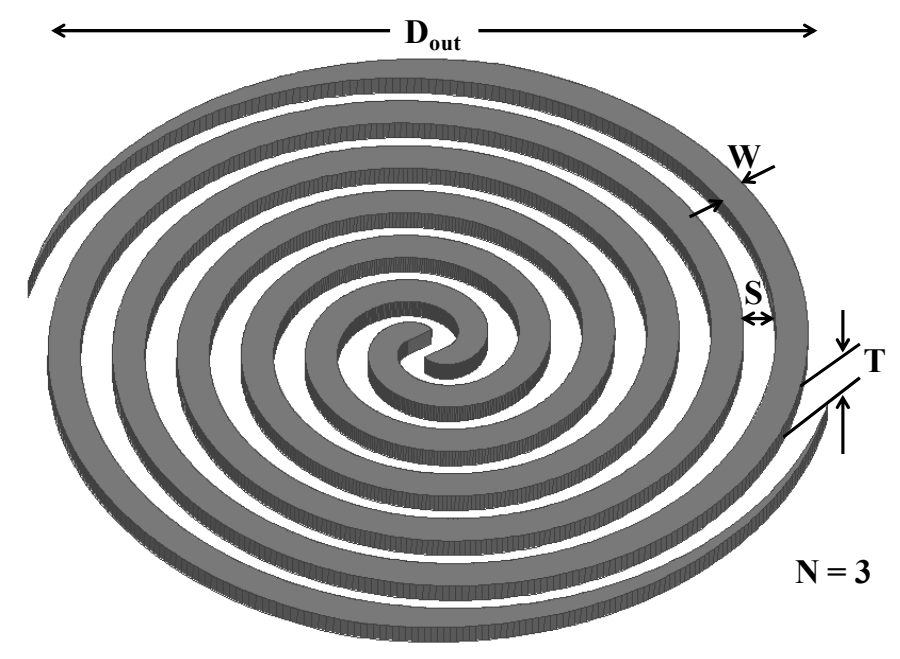

Figure 5.10: Bifilar spiral.

The resonant inductive coupling system of Figure 5.11(a) relies on the use selfresonant source and load elements (i.e., bifilar spirals) that are fed at their center. The SCMR system uses loops for the source and load elements and bifilar spirals for TX and RX resonators, as shown in Figure 5.11(b). Finally, the CSCMR consists of source and load loop elements, and bifilar TX and RX resonators. The bifilar TX and RX resonators are placed inside the source and load loops, respectively. It should be noted that the bifilar spirals used in the SCMR and CSCMR systems have their excitation gaps open since they are used as parasitic resonators. For both methods the source loop is connected to the power source and the load loop is connected to a $50 \mathrm{Ohm} \mathrm{load.} \mathrm{All} \mathrm{systems} \mathrm{are}$ designed to operate at $39.7 \mathrm{MHz}$.

The three systems of Figure 5.11 use the same two bifilar spirals, one in the air (external) and one in tissue (implanted). The implanted spirals are embedded at a depth of 
$2.6 \mathrm{~cm}$ in muscle tissue in a human phantom, as shown in Figure 5.12. The geometrical parameters of the external TX bifilar spiral are as follows: $d_{\text {out }}=98 \mathrm{~mm}, N=8, T=0.5$ $\mathrm{mm}, W=1.52 \mathrm{~mm}$, and $S=1.52 \mathrm{~mm}$. The geometrical parameters of the implanted RX bifilar spiral are as follows: $d_{\text {out }}=30 \mathrm{~mm}, N=9, T=0.3 \mathrm{~mm}, W=0.48 \mathrm{~mm}$, and $S=0.48$ $\mathrm{mm}$. The distances in Figure 5.11(b) are as follows: $\ell_{1}=5 \mathrm{~mm}, \ell_{2}=50 \mathrm{~mm}$ and $\ell_{3}=4$ mm. The SCMR system shown in Figure 5.11(b) also uses: (a) a source loop with radius of $30 \mathrm{~mm}$ and cross-sectional of $1 \mathrm{~mm}$, and (b) a load loop with radius of $15 \mathrm{~mm}$ and cross-sectional of $0.5 \mathrm{~mm}$. The CSCMR system shown in Figure 5.11(c) uses: (a) a source loop with radius of $55 \mathrm{~mm}$ and cross-sectional of $1 \mathrm{~mm}$, and (b) a load loop with radius of $17 \mathrm{~mm}$ and cross-sectional of $0.1 \mathrm{~mm}$. All elements in Figure 5.11 are assumed to be made from copper (i.e., $\sigma=5.8 \times 10^{7} \mathrm{~S} / \mathrm{m}$ ). In order to achieve miniaturization of the SCMR and CSCMR implanted RX resonator bifilar spirals, they were embedded in a dielectric with permittivity value of 10.2 . This can be implemented in practice by either stripline circuits on the appropriate substrate or by encapsulating the bifilar spirals inside an appropriate dielectric material. All simulations are performed using Ansoft HFSS and the top portion of the HFSS 3D human body model (see Figure 5.12).

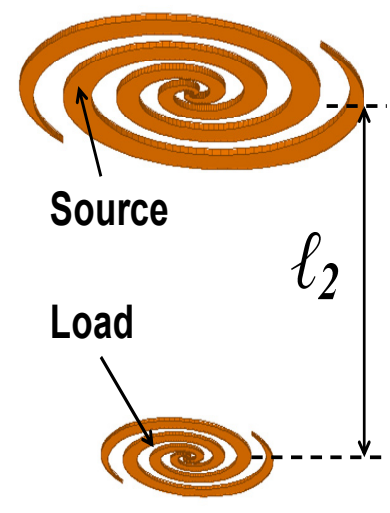

(a)

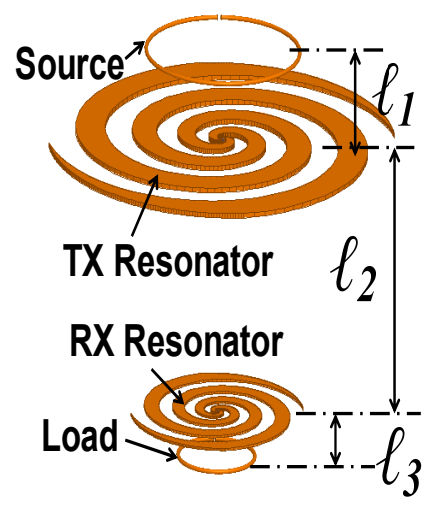

(b)

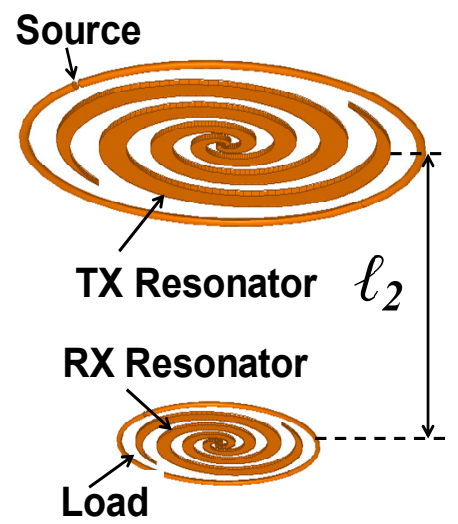

(c)

Figure 5.11: Models of three wireless powering systems. (a) Resonant inductive 
coupling, (b) SCMR, and (c) conformal SCMR (CSCMR).

Power requirements of IMDs depend on the specific applications, and the more efficient a wireless powering method is the less power needs to be used at the transmitter thereby decreasing the intensity of the generated fields as well as lowering the SAR. Therefore, it is expected that SCMR and CSCMR will outperform traditional resonant inductive coupling in terms of safety.

\subsubsection{Specific Absorption Rate (SAR) Analysis}

The conformal SCMR model is simulated using Ansoft HFSS and Nexxim with a $1 \mathrm{~W}$ input power. An output power of $385 \mathrm{~mW}$ was achieved at $\ell_{2}=50 \mathrm{~mm}$. In order to compare the three techniques for the same power delivered to the load, the input power of the resonant inductive coupling and the SCMR systems is adjusted so that an output power of $385 \mathrm{~mW}$ is delivered to the load. The performance of the three methods is compared in Table 5-3, where the input power, maximum H-field intensity, maximum SAR, and wireless powering efficiency are reported. As expected, it is clearly seen that SCMR and CSCMR significantly outperform resonant inductive coupling. Specifically, SCMR and CSCMR achieved an efficiency of $36.9 \%$ and $38.5 \%$, respectively, versus resonant inductive coupling that achieved an efficiency of only $0.46 \%$. This in turn means that resonant inductive coupling must utilize significantly greater amount of input power (i.e., 97 Watts) to achieve the same output power with SCMR and CSCMR. As a result, SCMR and CSCMR exhibit substantially lower levels of SAR (approximately 35 times lower maximum SAR) and magnetic field intensity. 


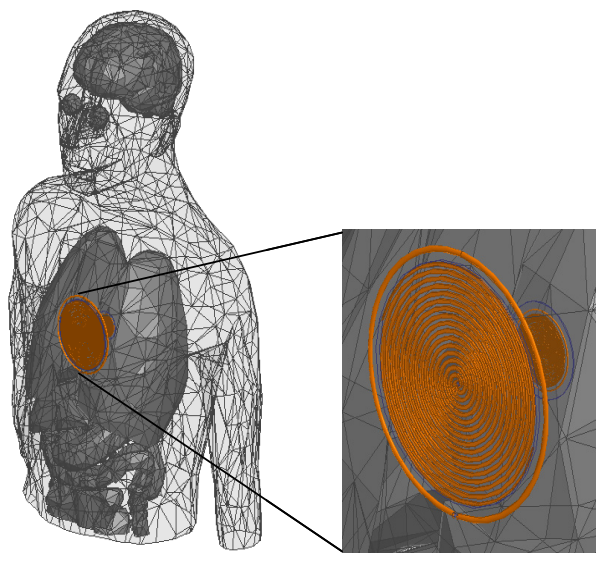

(a)

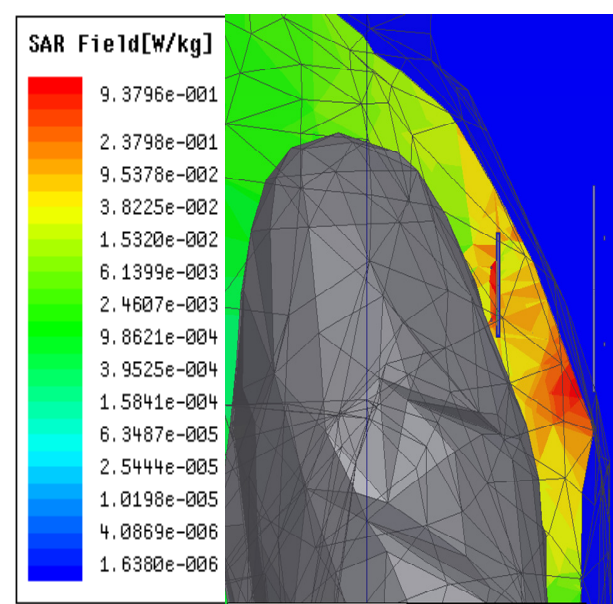

(b)

Figure 5.12: CSCMR in the human body. (a) Geometry of the human body, and (b) SAR distribution.

Therefore, SCMR and CSCMR are safer methods for wireless powering of implanted or wearable devices. Also, the SAR distribution for the CSCMR model is shown in Figure 5.11(b). In addition to achieving large efficiency and reducing SAR levels, CSCMR exhibits a compact size because it uses planar transmitter and receiver configurations thereby minimizing the volume required. Minimization of volume is particularly important for IMDs and biomedical applications. Therefore, CSCMR is very well suited for wireless powering of wearable and implantable devices. A comparison of the dimensions, volume and height of reported SCMR systems for biomedical applications is shown in Table 5-4.

Table 5-3. Comparison of three WPT methods

\begin{tabular}{|c|c|c|c|c|}
\hline \multirow{2}{*}{$\begin{array}{c}\text { Coupling } \\
\text { type }\end{array}$} & \multicolumn{4}{|c|}{ Field Parameters and Efficiency } \\
\cline { 2 - 5 } & $\begin{array}{c}\text { Input Power } \\
(W)\end{array}$ & $\begin{array}{c}\text { Max H-field } \\
(\mathrm{A} / \mathrm{m})\end{array}$ & $\begin{array}{c}\text { Max SAR } \\
(\mathrm{W} / \mathrm{kg})\end{array}$ & $\begin{array}{c}\text { Efficiency } \\
(\%)\end{array}$ \\
\hline Resonant & 97 & 50 & 35 & 0.46 \\
\hline SCMR & 1.3 & 5.6 & 0.98 & 36.9 \\
\hline CSCMR & 1 & 3.4 & 0.91 & 38.5 \\
\hline
\end{tabular}


Table 5-4. Comparison of different WPT systems for IMD applications

\begin{tabular}{|c|c|c|c|c|}
\hline Papers & $\begin{array}{c}\text { Dimension } \\
(r, H)(\mathrm{mm})\end{array}$ & Volume $(\mathrm{mm})^{3}$ & $\begin{array}{c}\text { Volume } \\
(\mathrm{mm})\end{array}$ & $\begin{array}{c}\text { Freq. of operation } \\
(\mathrm{MHz})\end{array}$ \\
\hline$[87]$ & $(85,50)$ & $1,134,900$ & 50 & 6.9 \\
\hline$[78]$ & $(11,2.5)$ & 950.3 & 10 & 0.7 \\
\hline SCMR & $(15,4)$ & $2,827.4$ & 4 & 39 \\
\hline CSCMR & $(15,0.35)$ & 247.4 & 0.35 & 39 \\
\hline
\end{tabular}

The data reported in Table 5-4 clearly illustrate that the proposed CSCMR system presented in this section requires very small volume and footprint.

\subsection{Wearable medical devices (WMD)}

Wearable devices are expected to play an important role in many applications, such as monitoring the health status of elderly patients or patients undergoing medical care at home. In addition, wearable devices can reduce cost and improve comfort [121]. They can also be used for tracking human body kinematics to allow clinicians to classify and analyze a stroke patient's progress, which aid in the rehabilitation plan [122]. A wearable medical device (WMD) is also used in high-risk cardiac and respiratory patients to monitor and alert as necessary, the system might include periodical or continuous collection and evaluation of multiple vital signs [123]. WMDs are saving and extending lives, dues to their ability to monitor, stimulate and regulate vital organs, and also communicate with the host about the state of health of the these organs intelligently. 


\subsubsection{Wireless Powering of Wearable Devices}

The design process for optimal SCMR spiral, which was presented in section 3.2.2 is used here to calculate the geometrical parameters of the TX and RX spirals in order to design an SCMR system for wearable devices. The specification of the models are: TX spiral model dimensions are $N=45, d_{i n}=4 \mathrm{~mm} d_{\text {out }}=272 \mathrm{~mm}, w=2 \mathrm{~mm}$ and the material is copper. The RX spiral model dimensions are $d_{\text {in }}=3.8 \mathrm{~mm} d_{\text {out }}=243 \mathrm{~mm}$, $\mathrm{w}=1.7 \mathrm{~mm}$. The distance between TX and RX spiral is varied from 10, 20 and $30 \mathrm{~cm}$. The RX spiral is $3 \mathrm{~mm}$ from the skin and embedded in a dielectric with a permittivity value of 4.2 to simulate clothing effect.

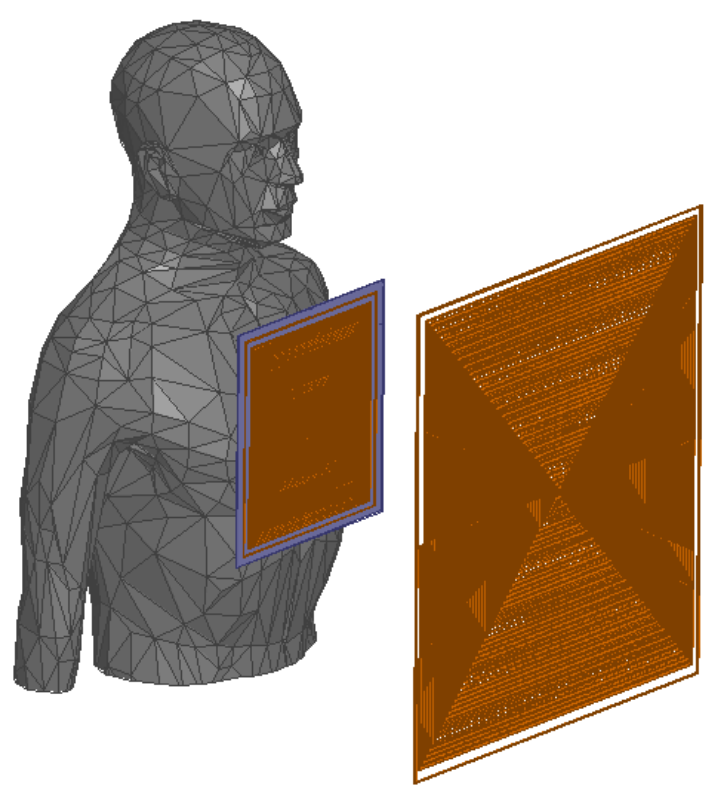

Figure 5.13: Wearable CSCMR system.

The models were simulated in HFSS with the human phantom model. Figure 5.13 shows the part of the human phantom included in our simulations with the planar SCMR system. The efficiency plots for the different parts of the body examined are shown in Figure 5.14 and Figure 5.15. 


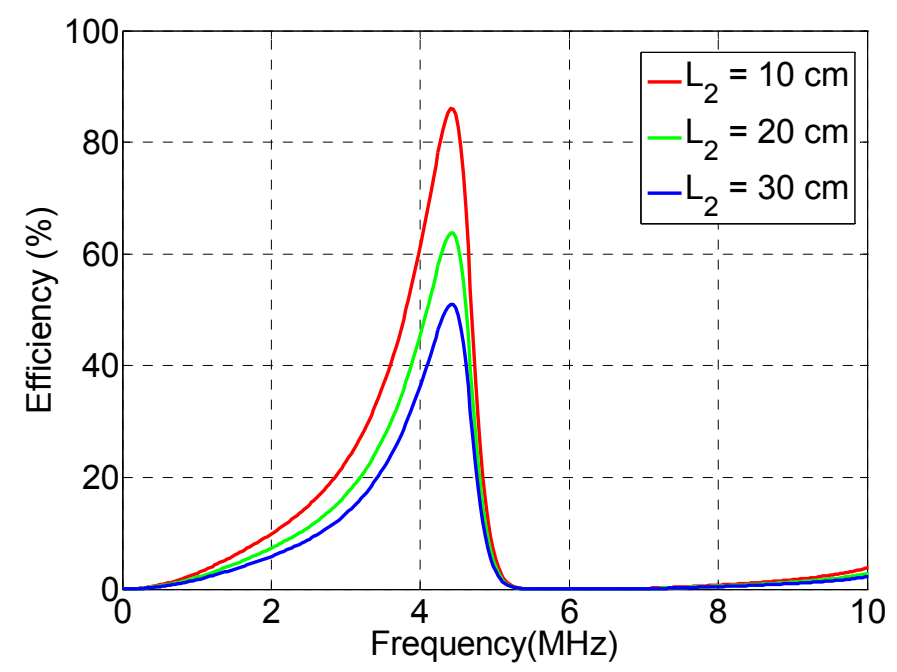

Figure 5.14: Efficiency plot human chest at $3 \mathrm{~mm}$ distance.

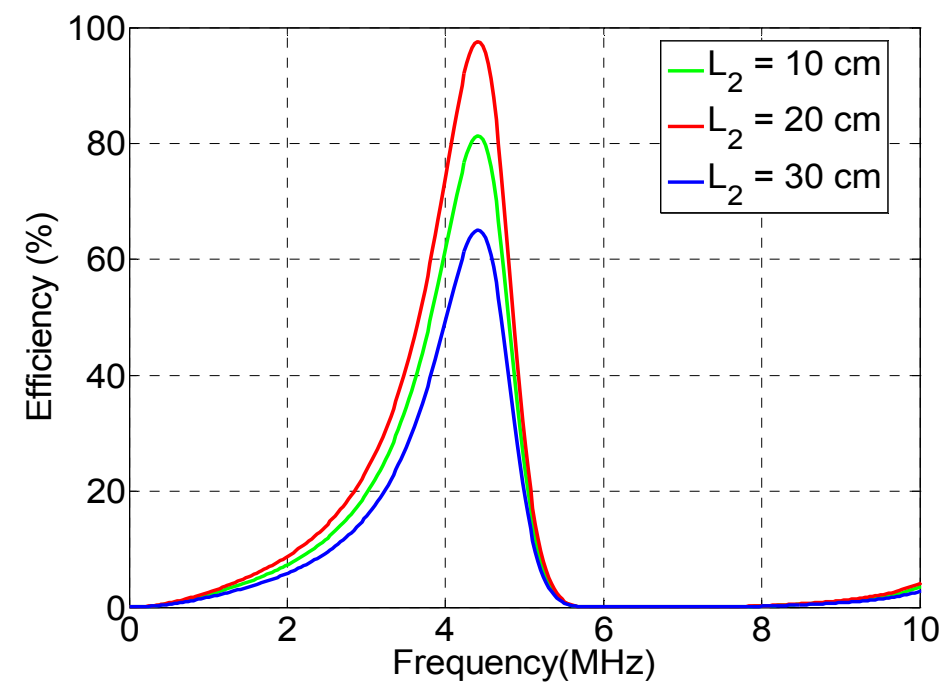

Figure 5.15: Efficiency plot human head at $3 \mathrm{~mm}$ distance.

\subsubsection{Specific Absorption Rate (SAR) Analysis}

The maximum SAR, along with the maximum magnetic and electric field intensity are reported in Figure 5.16, Tables 5-5 and 5-6 for two cases examined in the previous section. The result shows that the SCMR has great potentials in MWMD, and 
has low SAR, which is important for biomedical applications. Also, the SCMR geometry that was presented here is planar and therefore can be embedded in clothing easily.

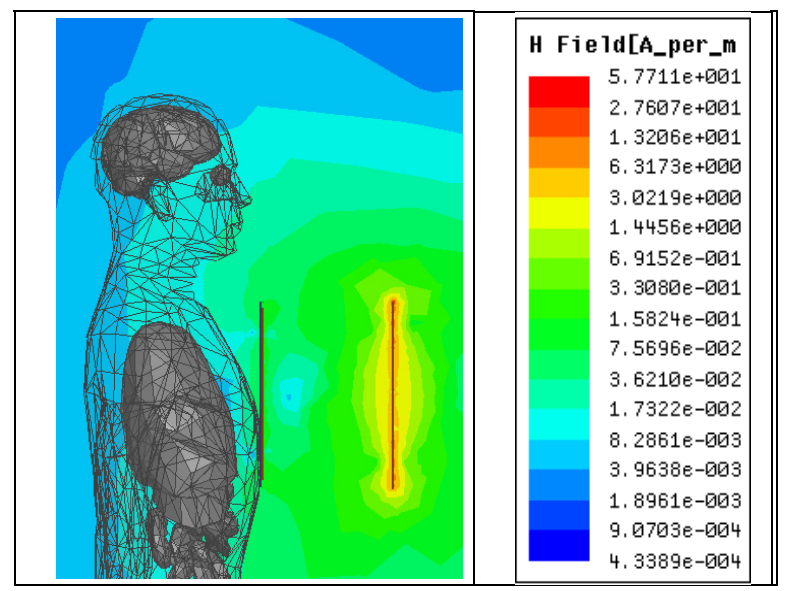

Figure 5.16: Magnetic field distribution in the chest distance of $20 \mathrm{~cm}$.

Table 5-5. CSCMR system at $3 \mathrm{~mm}$ from chest ( 1 Watt input power)

\begin{tabular}{|c|c|c|c|c|}
\hline \multirow{2}{*}{ Distance (cm) } & \multicolumn{4}{|c|}{ Field Parameters and Efficiency } \\
\cline { 2 - 5 } & $\begin{array}{c}\text { Max E-field } \\
(\mathrm{V} / \mathrm{m})\end{array}$ & $\begin{array}{c}\text { Max H-field } \\
(\mathrm{A} / \mathrm{m})\end{array}$ & $\begin{array}{c}\text { Max SAR } \\
(\mathrm{W} / \mathrm{kg})\end{array}$ & $\begin{array}{c}\text { Efficiency } \\
(\%)\end{array}$ \\
\hline 10 & 18 & 0.21 & 0.07 & 83 \\
\hline 20 & 12 & 0.08 & 0.0004 & 64 \\
\hline 30 & 0.9 & 0.004 & 0.00008 & 56 \\
\hline
\end{tabular}

Table 5-6. CSCMR system at $3 \mathrm{~mm}$ from the head ( 1 Watt input power)

\begin{tabular}{|c|c|c|c|c|}
\hline \multirow{2}{*}{$\begin{array}{c}\text { Distance } \\
(\mathrm{cm})\end{array}$} & \multicolumn{4}{|c|}{ Field Parameters and Efficiency } \\
\cline { 2 - 5 } & $\begin{array}{c}\text { Max E-field } \\
(\mathrm{V} / \mathrm{m})\end{array}$ & $\begin{array}{c}\text { Max H-field } \\
(\mathrm{A} / \mathrm{m})\end{array}$ & $\begin{array}{c}\text { Max SAR } \\
(\mathrm{W} / \mathrm{kg})\end{array}$ & $\begin{array}{c}\text { Efficiency } \\
(\%)\end{array}$ \\
\hline 10 & 15 & 0.15 & 0.0005 & 92 \\
\hline 20 & 5 & 0.05 & 0.00001 & 81 \\
\hline 30 & 1.2 & 0.001 & 0.000002 & 63.5 \\
\hline
\end{tabular}




\subsection{Summary}

The chapter illustrated that SCMR and CSCMR techniques are more efficient than resonant inductive coupling for wearable and implantable devices. However, more work is required to further reduce the size of the embedded sensor in the tissues and increase the range of the embedded sensor. A new conformal SCMR method (CSCMR) was introduced that achieves high efficiency and minimizes the volume of wireless powering systems. High efficiency enables CSCMR to deliver a significant amount of power to IMDs without excessive transmitter power. In fact, CSCMR outperformed resonant inductive coupling and performed similarly to SCMR in terms of the SAR levels for the same power delivered. CSCMR is also planar and therefore, it is more suitable for planar and flexible implantable or wearable circuits. Due to these important features, CSCMR is very well suited and promising technique for wireless powering of implantable and wearable devices. The result shows that the SCMR has great potentials in WIMD, and has very low SAR, which is important for biomedical applications. 


\section{CHAPTER 6}

\section{SIMULTANEOUS WIRELESS POWERING AND COMUNICATION IN RFID APPLICATIONS}

Simultaneous wireless powering and data transfer is beneficial for applications such as, passive RFID tags. An important advantage of SCMR over resonant inductive coupling which is currently used in near-field RFID, is the ability to wirelessly transfer power in the mid-range with high efficiency [10]. Even though simultaneous wireless power and data transfer has been achieved by resonant inductive coupling, it has never been attempted with the SCMR. An AC-powered RFID transponder using shadow-masks were developed in [124]. The system operated at $8.8 \mathrm{MHz}$ frequency, and created a pulse signal by powering directly from the RF energy absorbed from the resonant LC tank. An on-chip integrated RFID system, which is designed and fabricated was developed in [125]. The system operates at $900 \mathrm{MHz}$ with the coverage range of more than $0.4 \mathrm{~cm}$. An RFID system with reflect-array antenna, which can work at $2.4 \mathrm{GHz}$ and produce near-field focused patterns in a target area was presented in [126]. The design has less complexity than phased array antennas in the beam-forming. An RFID antenna was presented in [127] which is composed of two printed dipoles with dimension of $80 \times 70 \times 30 \mathrm{~mm} 3$. The antenna can provide in-phase current performance. The measured reading range was 37 $\mathrm{mm}$, with voltage standing wave ratio less than 1.5 , and far-field gain less than $-20 \mathrm{dBi}$. The RFID tag antenna that was presented in [128] can work at both HF and UHF, the UHF antenna was embedded inside the HF coil to save the space, and the size of whole 
antenna is similar to a credit card. The UHF antenna consists two meander lines and set as the diagonal symmetric to enlarge the UHF bandwidth.

In this chapter, the SCMR method will be used to develop a passive RFID system. This system is expected to have greater range than the range of conventional inductive resonant coupling RFID system [10]-[13] due to the SCMR's high efficiency. However, SCMR system exhibits less bandwidth that inductive coupling system. This problem will be mitigated by developing a novel SCMR system that can provide both high efficiency and sufficient bandwidth.

\subsection{Comparison of WPT Methods}

In this section, SCMR will be compared with resonant inductive coupling using single turn loops. Figure 6.1(a) shows the resonant inductive coupling system used in RFID. Figure 6.1(b) shows an SCMR system. In Figure 6.1(a) a capacitor is connected across each of source and load loops to form a resonant circuit and with low efficiency. In contrast, in the SCMR system of Figure 6.1(b) the source and load loops are inductively coupled to the RX and TX loop resonant circuits, and the TX and RX loops have high Q-factors resulting in high wireless power transfer efficiency.

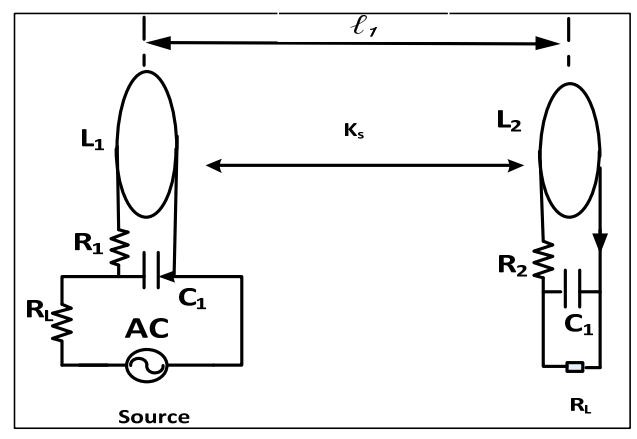

(a) 


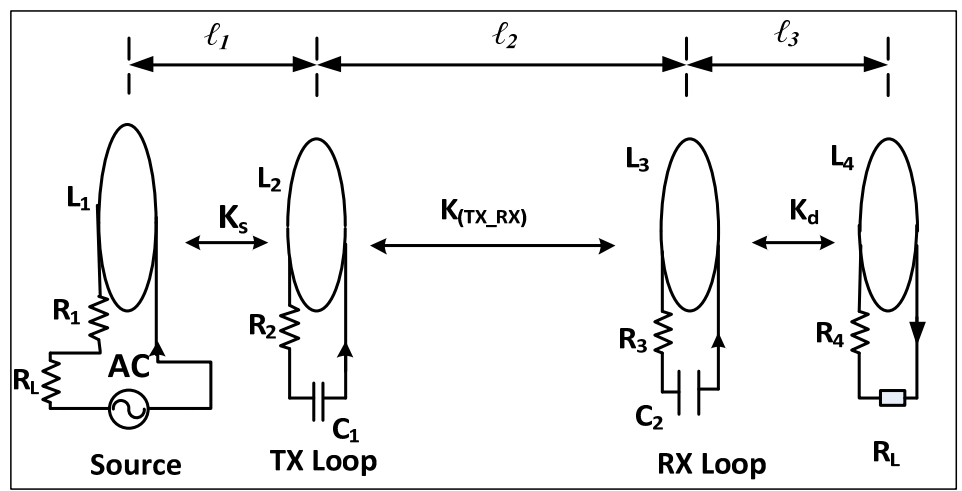

(b)

Figure 6.1: Inductive coupling systems: (a) RFID resonant coupling with loading from source and load loops. (b) SCMR resonant coupling with source and load inductively coupled to the resonant circuit. $\left(\ell_{1}=\ell_{3}=50 \mathrm{~mm}\right.$ and $\left.\ell_{2}=200 \mathrm{~mm}\right)$.

The systems of Figure 6.1 were simulated in Ansoft Designer and measured in the lab with the following parameters: loop radius, $r=100 \mathrm{~mm}$, cross-sectional radius, $r_{c}=$ $2.1 \mathrm{~mm}, \ell_{1}=\ell_{3}=50 \mathrm{~mm}$ and $\ell_{2}=200 \mathrm{~mm}$, the capacitance $\mathrm{C}=10 \mathrm{pF}$. Figure 6.3 compares the efficiencies of the system. Figure 6.3 shows the loop-based SCMR wireless power transfer system measurement setup. The efficiency is approximately $9.5 \%$ and $58.7 \%$ for the resonant inductive coupling and SCMR respectively.

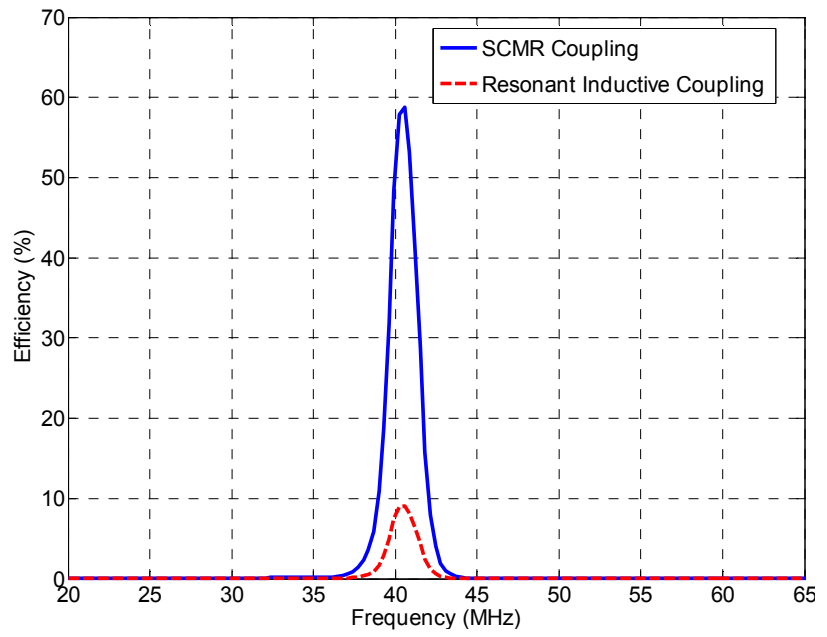

Figure 6.2: Comparison of the efficiency of the resonant and SCMR coupling. 
Therefore, even though the SCMR system of Figure 6.2 (b) has a larger efficiency than the resonant inductive coupling, it will be challenging transferring data through the SCMR system's because of its narrow bandwidth. In what follows, we will explain the novel SCMR system that can achieve sufficient bandwidth to sustain data transfer, while maintaining large wireless powering efficiency.

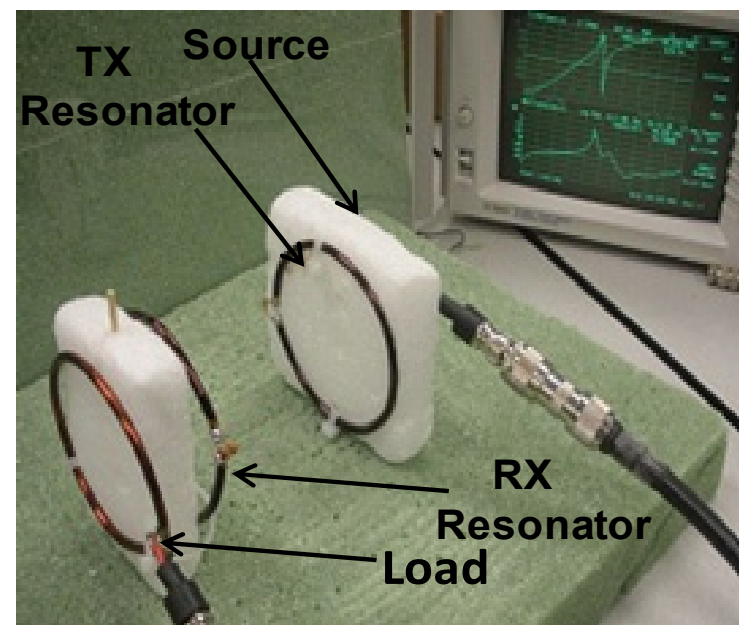

Figure 6.3: SCMR system measurement setup.

\subsection{Wireless Powering with Resonant Inductive Coupling}

Wireless power and data transfer (WPDT) by the RFID or SCMR system are functions of their geometrical and electrical properties. From the equivalent RLC circuit of Figure 1, the resonant frequency, $f_{r}$, is usually calculated from (3.1). In this loops are used and hence in the loops, which will have negligible self-capacitance, in order to achieve resonance in the loops, it must be connected to a capacitor in order to form a resonant series RLC circuit and the capacitance can be calculated from (3.1). Furthermore, in order to achieve an optimal design, the RFID and SCMR devices must be 
optimized for Q-factor that will have sufficient bandwidth to transfer data at the specified RFID frequency.

\subsubsection{RFID}

The simple RFID reader and tag consist of two loops (each connected to the source and load respectively), the loaded Q-factor at load and source for a typical two device system can be written as [92]:

$$
Q\left(R_{2}, R_{L}, L_{2}\right)=\frac{1}{\left(\frac{R_{2}}{\omega L_{2}}\right)+\left(\frac{\omega L_{2}}{R_{L}}\right)}
$$

For the loop-based RFID system, from (3.2) to (3.4) this can also be written as:

$$
Q\left(R_{2}, R_{L}, L_{2}\right)=\left(\frac{\omega \mu_{0} r \ln \left(\frac{8 r}{r_{c}}-2\right)}{\left(\frac{\mu_{0} \rho \omega r^{2}}{2 r_{c}^{2}}\right)^{\frac{1}{2}}+20 \pi^{2}\left(\frac{\omega r}{c}\right)^{4}}+\frac{R_{L}}{\omega \mu_{0} r \ln \left(\frac{8 r}{r_{c}}-2\right)}\right)^{-1}
$$

where $L$ and $C$ are the self-inductance and resonating capacitance. The other basic parameters of the loops are $R_{2}, R_{L} R_{\text {rad }}$ and $R_{\text {ohm }}$ which are the source, load radiation and ohmic resistance of the respective loops [94], $\mu$ is the permeability of free space, $\rho$ is the loop's material resistivity, $r$ is the radius of the loop, $r_{c}$ is the cross sectional wire radius, $\eta_{o}$ is the impedance of free space, and $c$ is the speed of light. The bandwidth, $B W$ of the RFID resonant system circuit is inversely proportional to the Q-factor and can be calculated as [94], [80]:

$$
B W=\frac{f_{r}}{Q}=\frac{f_{r}}{Q\left(R_{2}, R_{L}, L_{2}\right)}
$$




\subsubsection{SCMR}

In an SCMR system, there are two types of Q-factor namely, loaded and the unloaded Q-factor. The unloaded Q-factors exist in the inductively coupled TX and RX loop resonators (which are not connected to any device, except a capacitor to achieve resonance at the desired frequency in loops) (see Figure 6.3), while the loaded Q-factors occur in the load and source loops. The loaded Q-factors in an SCMR system are identical to the ones of an RFID system described by (6.1) - (6.3). The unloaded Q-factor of a resonant TX and RX loops can be expressed in terms of their geometrical parameters using (3.9)-(3.11). The maximum Q-factor, $Q_{\max }$, and the frequency, $f_{\max }$, where $Q_{\max }$ occurs, can be calculated from (3.10) and (3.11) respectively. For instance, if $r=100$ $m m, r_{c}=2.2 \mathrm{~mm}$ and $1 \mathrm{KHz} \leq f \leq 100 \mathrm{MHz}$, we can plot the Q-factor as shown in Figure 6.4. Hence, the maximum Q-factor $\left(Q_{\max }\right)$ and the corresponding frequency, $f_{\max }$, where $Q_{\max }$ occurs can be easily obtained from (3.10) and (3.11) are as shown in Figure 6.4.

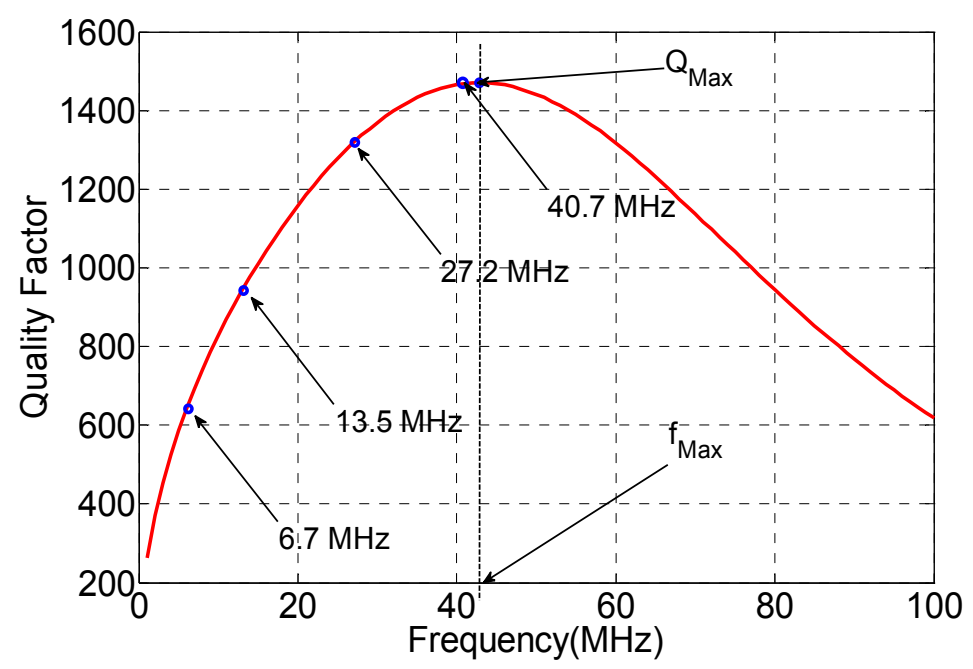

Figure 6.4: Q-factor of SCMR TX and RX resonators. 
Generally, SCMR systems require that both RX and TX loops are resonant where they exhibit maximum Q-factor, in order to achieve maximum wireless power efficiency. However, this is a major drawback for applications that requires simultaneous data transfer, because of the inverse relationship between bandwidth and Q-factor. Three approaches are proposed in order to improve the bandwidth in the SCMR system significantly. The first approach involves resonating and operating the RX and TX loops of at a resonant frequency that is lower than the $f_{\max }$, thereby exhibiting a Q-factor much lower than $Q_{\max }$ (see Figure 6.4), and in turn increasing the bandwidth significantly. However, this approach leads to an SCMR system with lower efficiency and range. Figure 6.4 shows some applicable RFID frequencies at which the SCMR system could be operated.

Table 6.1, which is extracted from Figure 6.5, shows the various Q-factors and the respective bandwidth of the loop at different RFID frequencies. Table 6.1 shows that the bandwidth of the TX and RX loops calculated from (6.2) and (6.3), is not sufficient as required by RFID applications at these RFID frequencies, while the bandwidth of the source and load loops is sufficient.

Table 6.1 Q-factor and bandwidth of SCMR elements.

\begin{tabular}{|c|c|c|c|c|}
\hline & \multicolumn{2}{|c|}{ TX and RX Loops } & \multicolumn{2}{c|}{ Source and Load Loops } \\
\hline $\begin{array}{c}\text { Frequency } \\
(M H z)\end{array}$ & Q-factor & $\begin{array}{c}\text { Bandwidth } \\
(\mathrm{kHz})\end{array}$ & Q-factor & $\begin{array}{c}\text { Bandwidth } \\
(\mathrm{kHz})\end{array}$ \\
\hline $43.5\left(f_{\max }\right)$ & $1520\left(Q_{\max }\right)$ & 29.6 & 3.8 & 11,447 \\
\hline 40.7 & 1420 & 30.9 & 4.3 & 9,465 \\
\hline 27.2 & 1310 & 20.8 & 6.9 & 3,942 \\
\hline 13.5 & 950 & 14.2 & 12.2 & 1,150 \\
\hline 6.8 & 670 & 10.2 & 25.0 & 272 \\
\hline
\end{tabular}


The second approach is to design an SCMR system with the lower Q-factor than the model in Figure 6.3, such that it exhibit high bandwidth at the desired frequencies. This modification was done on the model of Figure 6.3 with the following specifications : $r=100 \mathrm{~mm}, r_{c}=0.05 \mathrm{~mm}$ and $1 \mathrm{KHz} \leq f \leq 100 \mathrm{MHz}$. Figure 6.5 shows the Q-factor of the modified system. In addition, Table 6.2 shows the Q-factors and their respective bandwidth of the four identical loops calculated from (6.2), (6.3). The bandwidth of the TX and RX loops does not lead to sufficient bandwidth required by RFID applications due to their high Q-factor, but the bandwidth of the source and load loops is sufficient,because of their low Q-factor. These results also show that the approach does not lead to sufficient bandwidth for RFID applications, at the cost of significantly reduced efficiency.

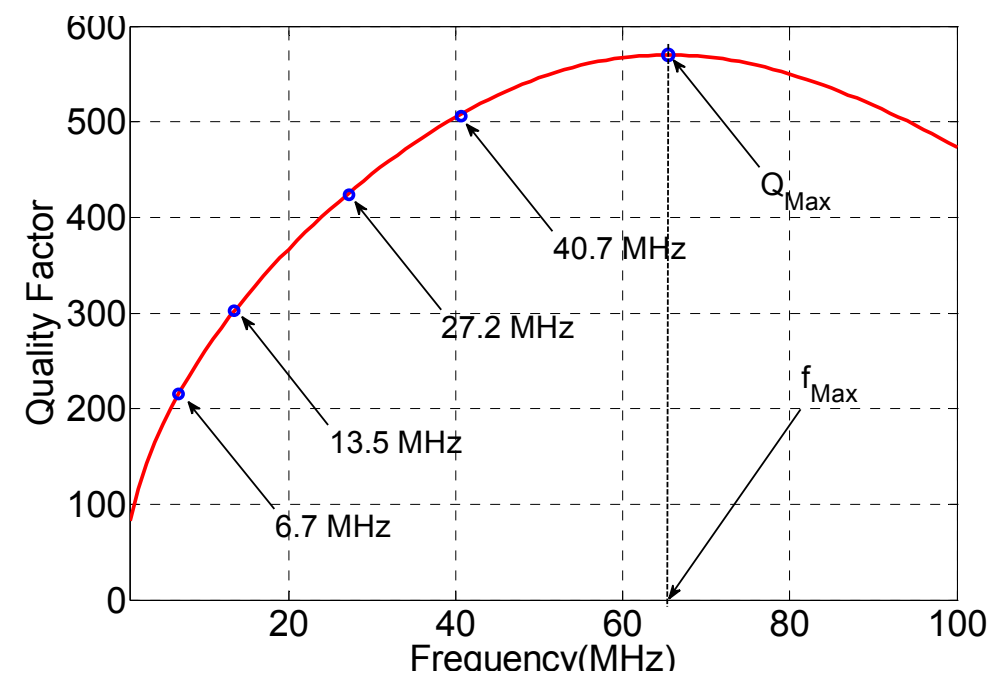

Figure 6.5: Q-factor of modified TX and RX resonator. 
Table 6.2 Q-factor and bandwidth of SCMR elements.

\begin{tabular}{|c|c|c|c|c|}
\hline & \multicolumn{2}{|c|}{ TX and RX loops } & \multicolumn{2}{c|}{ Source and Load loops } \\
\hline $\begin{array}{c}\text { Frequency } \\
(\mathrm{MHz})\end{array}$ & $\begin{array}{c}\text { Q-factor } \\
\text { Bandwidth } \\
(\mathrm{KHz})\end{array}$ & $\begin{array}{c}\text { Q-factor } \\
(\mathrm{KHz})\end{array}$ \\
\hline $65.5\left(f_{\max }\right)$ & $570\left(Q_{\max }\right)$ & 115 & 3.8 & 11,447 \\
\hline 40.7 & 506 & 80.5 & 4.3 & 9,465 \\
\hline 27.2 & 424 & 64.2 & 6.9 & 3,942 \\
\hline 13.5 & 296 & 45.6 & 12.2 & 1,150 \\
\hline 6.8 & 201 & 33.8 & 25.0 & 272 \\
\hline
\end{tabular}

\subsubsection{Broadband SCMR}

The third approach is based on a novel SCMR system that uses a multiple TX and $\mathrm{RX}$ resonating loops and resonates lower frequency than the $f_{\max }$ and the Q-factor lower than $Q_{\max }$, such that it exhibit bandwidth sufficient for power and data transfer. This modification was done on the model in Figure 6.3 with the following specification : $r_{l}=$ $30 \mathrm{~mm}, r_{2}=50 \mathrm{~mm}, r_{3}=65 \mathrm{~mm}$, while the distance between adjacent loops is $20 \mathrm{~mm}, r_{c}$ $=2.2 \mathrm{~mm}$, capacitance $\mathrm{C}_{1}=10 \mathrm{pF}$ and $\mathrm{C}_{2}=18 \mathrm{pF}$ for $1 \mathrm{KHz} \leq f \leq 100 \mathrm{MHz}$, the plot the Q-factor as shown in Figure 6.6. The broadband has both high and low Q-factor with high coupling coefficient (alternated loop and source) to support efficient power transfer and data rate transfer. Table 6.3 shows the Q-factors and the respective bandwidth calculated from (6.2), (6.3) for the four loop resonators. The bandwidth of the loops $r_{1}$ does lead to sufficient bandwidth at $40.7 \mathrm{MHz}$ as required by RFID applications, due to their low Q-factor. The loops $r_{2}$ still exhibit low bandwidth and only useful for WPT. 


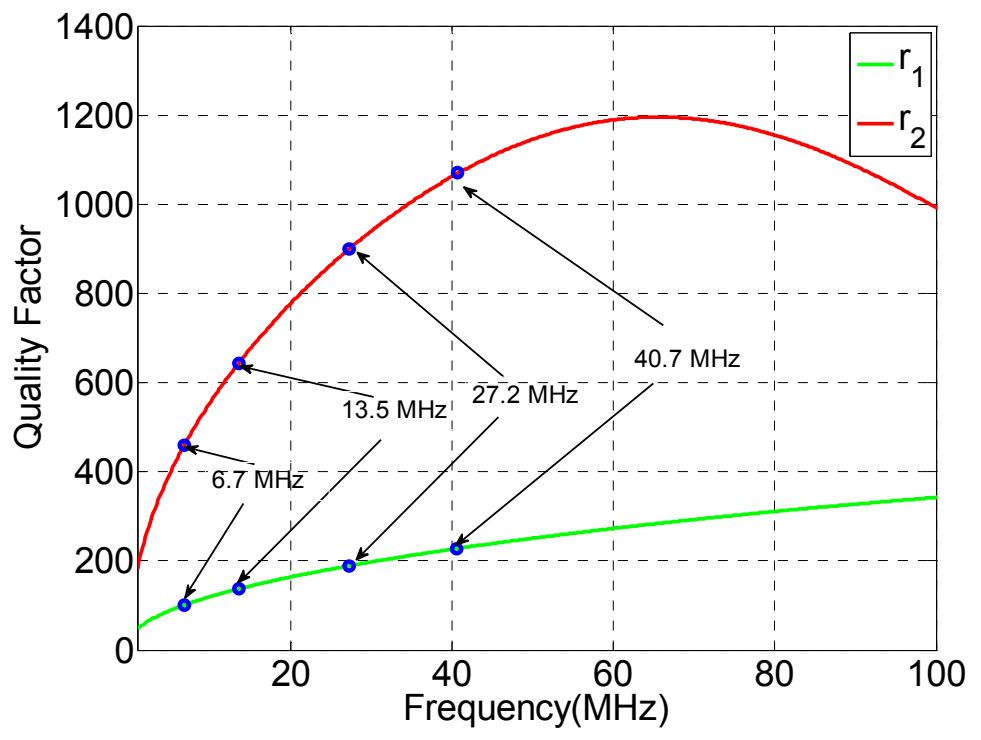

Figure 6.6: Q-factor of modified TX and RX resonator.

Table 6.3 Q-factor and bandwidth of SCMR elements.

\begin{tabular}{|c|c|c|c|c|}
\hline & \multicolumn{2}{|c|}{$\mathrm{R}_{1}$ loops } & \multicolumn{2}{c|}{$\mathrm{R}_{2}$ loop } \\
\hline $\begin{array}{c}\text { Frequency } \\
(\mathrm{MHz})\end{array}$ & Q-factor & $\begin{array}{c}\text { Bandwidth } \\
(\mathrm{KHz})\end{array}$ & $\begin{array}{c}\text { Q-factor } \\
(\mathrm{KHz})\end{array}$ \\
\hline 40.7 & 210 & 203.2 & 1080 & 37.7 \\
\hline 27.2 & 195 & 139 & 900 & 30.3 \\
\hline 13.5 & 170 & 80 & 650 & 20.1 \\
\hline 6.8 & 90 & 85 & 470 & 14.5 \\
\hline
\end{tabular}

\subsection{Simulation Results}

The design of the three approaches is simulated in HFSS and Nexxim. The three designs are the standard RFID, standard SCMR and SCMR with multiple loops [114], The three models are shown in Figure 6.7. The efficiency plot for the three cases is shown in Figure 6.8. 


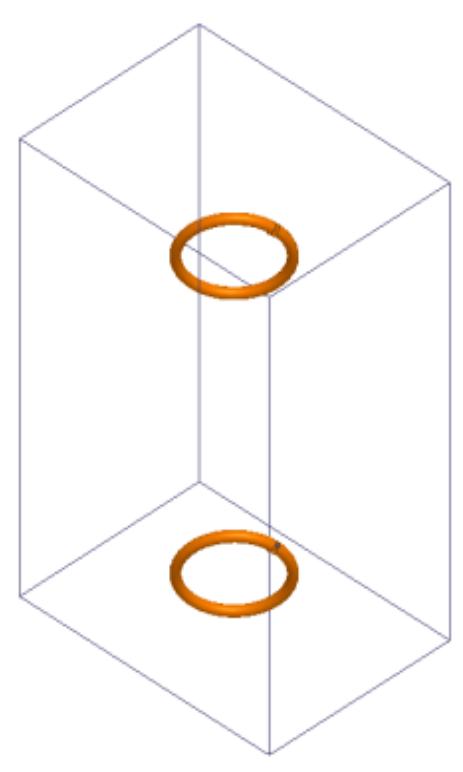

(a)

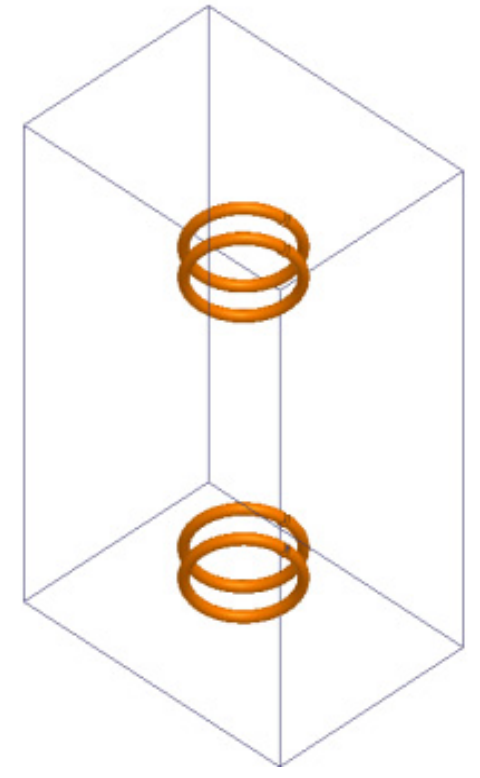

(b)

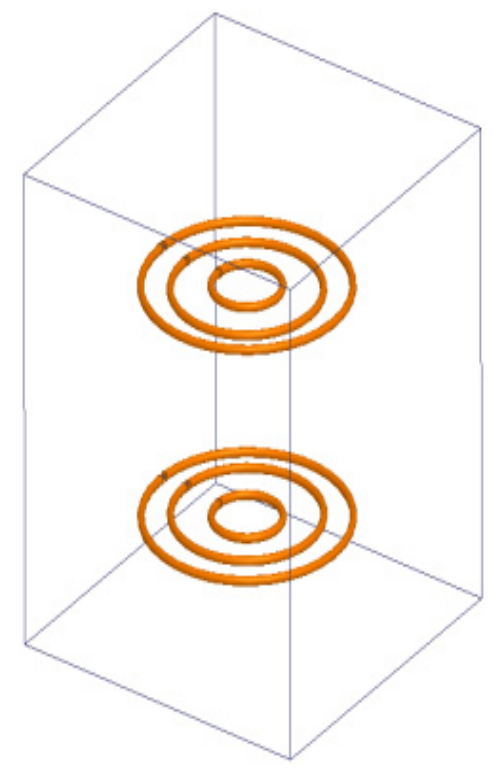

(c)

Figure 6.7: The three models (a) Standard RFID, (b) standard SCMR and (c) the broadband (multiband) SCMR.

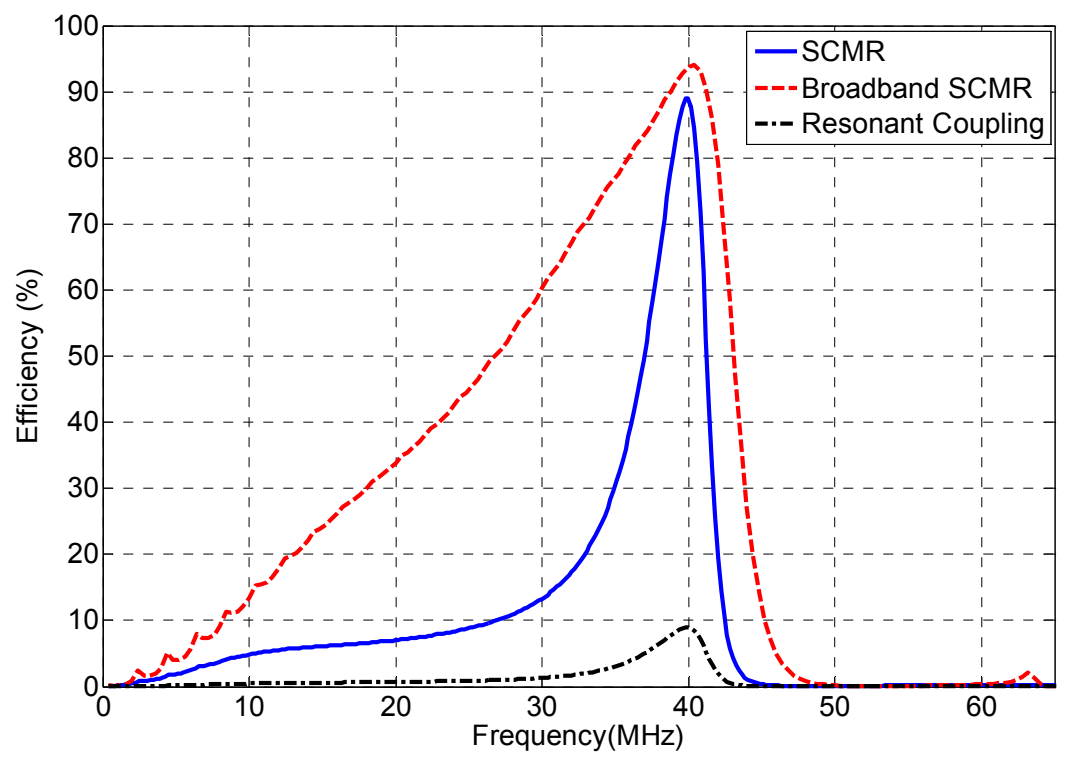

Figure 6.8: The efficiency plot of the three models at $\ell_{3}=100 \mathrm{~mm}$.

Furthermore, it is assumed that the message is " 1 " followed by " 0 " in a continuous stream and operating at $40.7 \mathrm{MHz}$ ISM band. Simulations are set up for the three 
systems of Figure 6.7 for backscattering modulation. When the three models were simulated with high bit rate (100 kbps), only the RFID and SCMR setup did not result in over-modulation because of their large bandwidth. However, when the bit rate was reduced to $15 \mathrm{kbps}$ and bandwidth is $30 \mathrm{kHz}$, the three models modulated tag and backscattering at the reader did not result in over modulation. The result of the high and low bandwidth are shown separately in the following sub-sections.

\subsubsection{Modulation and data transfer (High Bandwidth)}

In this case, the three models were simulated with high bit rate (100 kbps), only the RFID and SCMR (broadband) system did not result in over modulation because of their larger bandwidth.

\subsubsection{RFID}

The result of the RFID in the proposed design is as shown in Figure 6.9 and Figure 6.10 for the reader and tag respectively. The result shows that the tag can backscatter the message from the tag to the reader succesfully, hence can transfer data. 


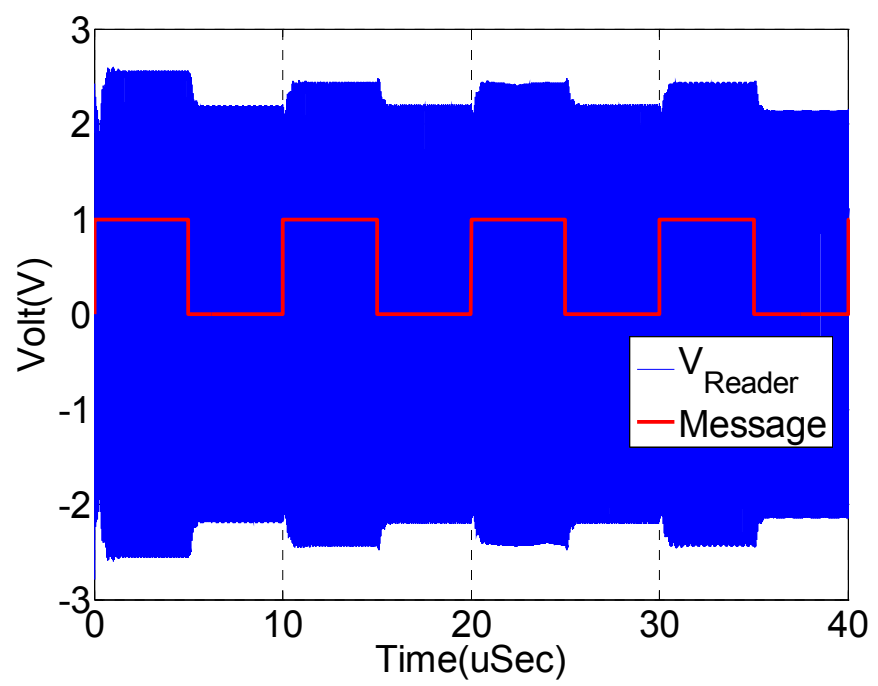

Figure 6.9: RFID with modulation at the reader.

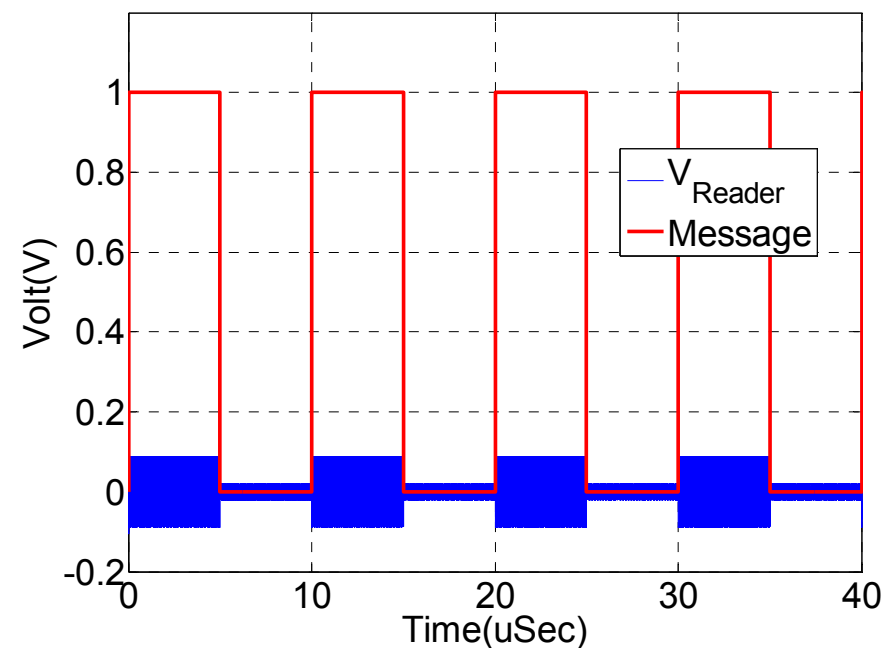

Figure 6.10: RFID with modulation at the tag.

\subsubsection{Standard SCMR}

The result of the SCMR in the proposed design is as shown in Figure 6.11 and Figure 6.12 for the reader and tag respectively. The result shows that modulated backscattering from the tag to the reader does not occurs, hence cannot transfer data. 


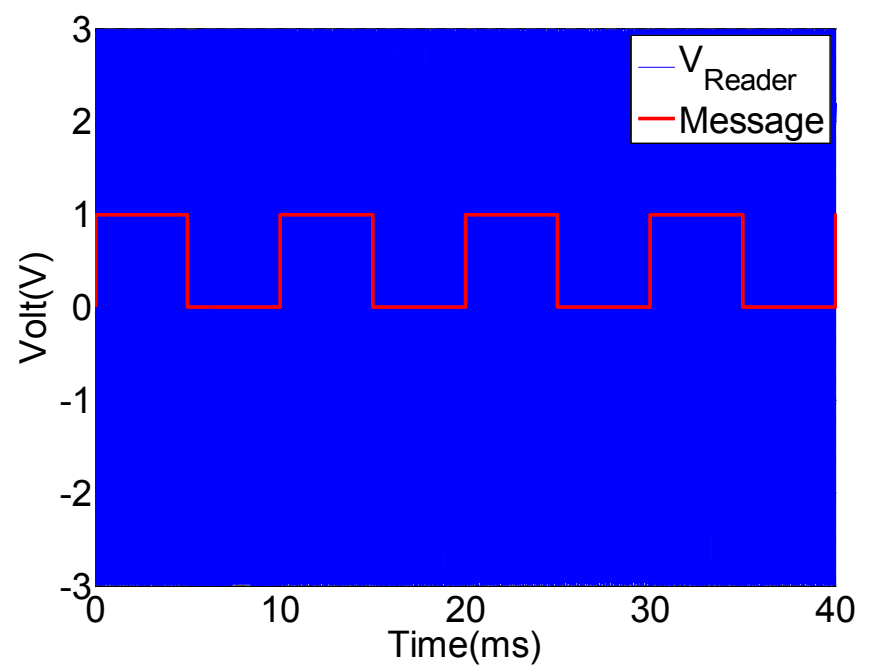

Figure 6.11: Standard SCMR with modulation at the reader.

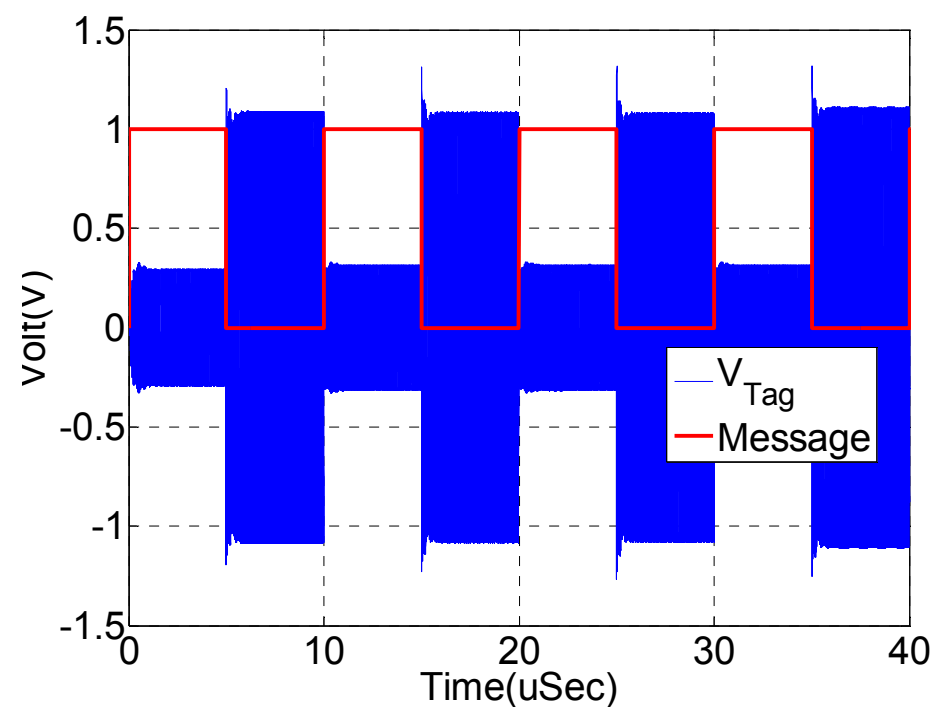

Figure 6.12: Standard SCMR with modulation at the tag.

\subsubsection{Broadband SCMR}

The result of the broadband SCMR in the proposed design is as shown in Figure 6.13 and Figure 6.14 for the reader and tag respectively. The result shows that modulated backscattering from the tag to the reader occurs, therefore can transfer data. 


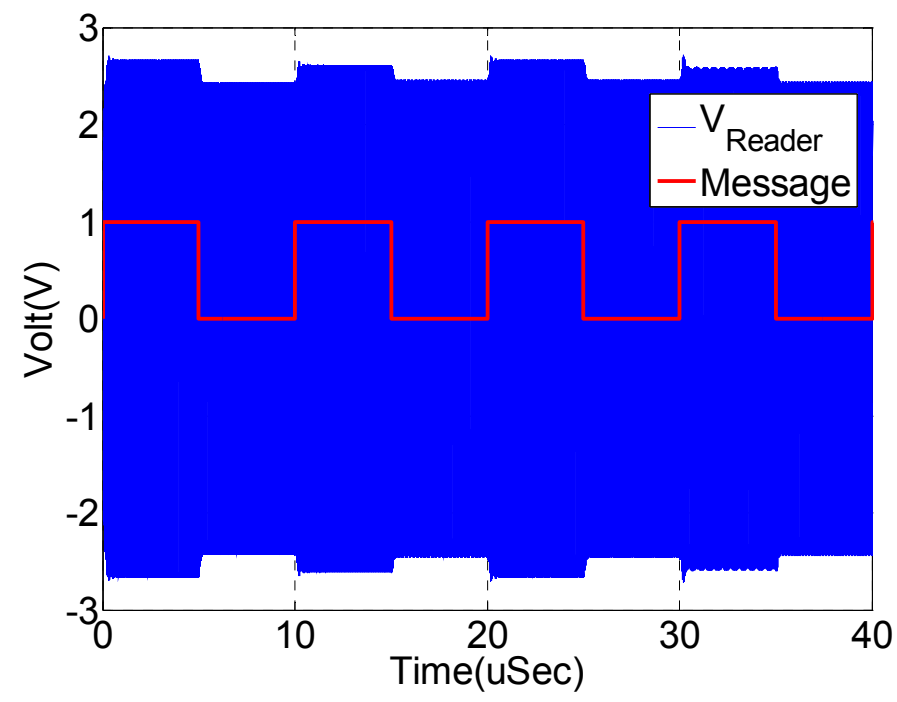

Figure 6.13: Broadband SCMR with modulation at the reader.

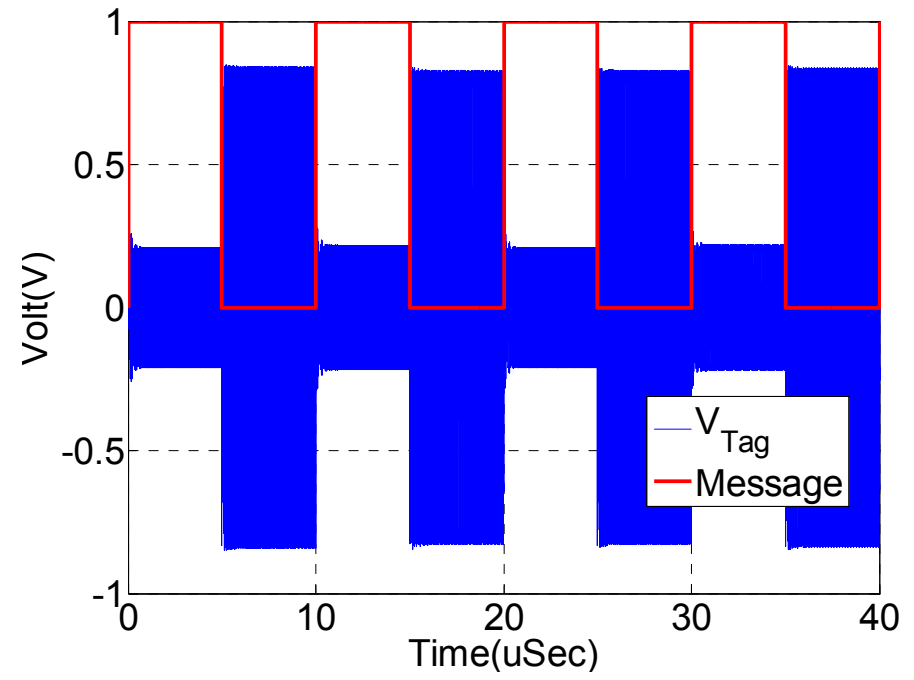

Figure 6.14: Broadband SCMR with modulation at the tag.

\subsubsection{Modulation and data transfer (Low Bandwidth)}

In this case, the three models were simulated with low bit rate $(15 \mathrm{kbps})$, the three system, RFID, standard SCMR and broadband SCMR system did not result in overmodulation because of the low bit rate, which only requires low bandwidth for operation. 


\subsubsection{RFID}

The result of the RFID in the proposed design is as shown in Figure 6.15 and Figure 6.16 in the reader and tag respectively. The result shows that modulated backscattering from the tag to the reader occurs.

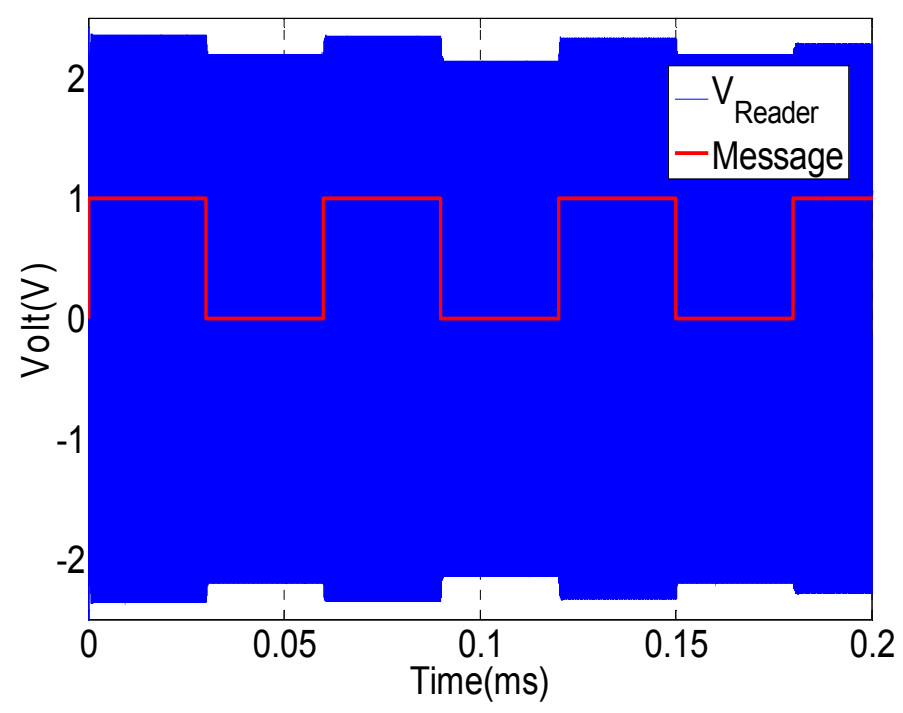

Figure 6.15: RFID with modulation at the reader.

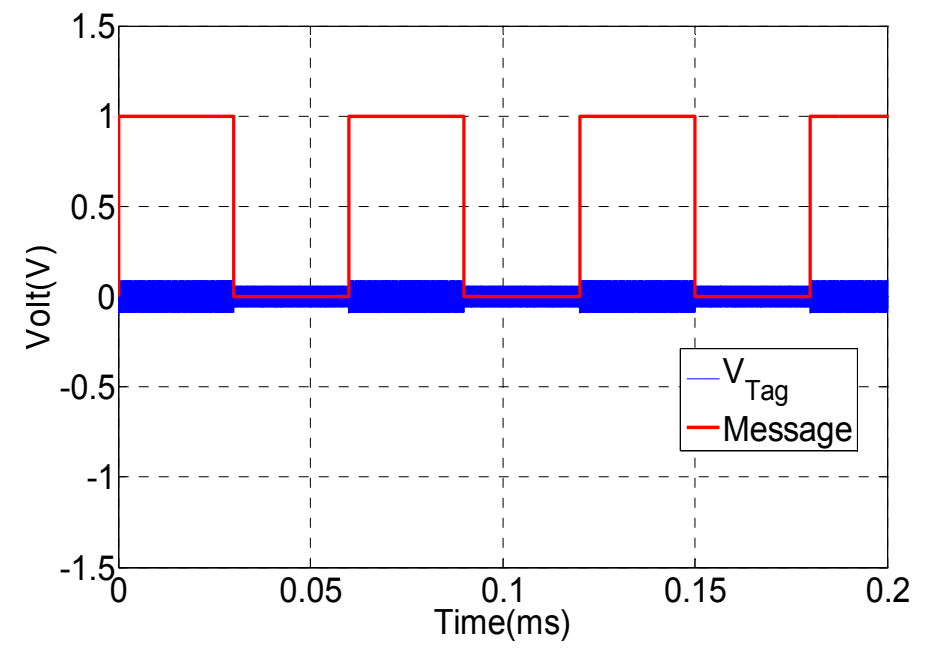

Figure 6.16: RFID with modulation at the tag. 


\subsubsection{Standard SCMR}

The result of the Standard SCMR in the proposed design is as shown in Figure 6.17 and Figure 6.18 in the reader and tag respectively. The result shows that modulated backscattering from the tag to the reader occurs.

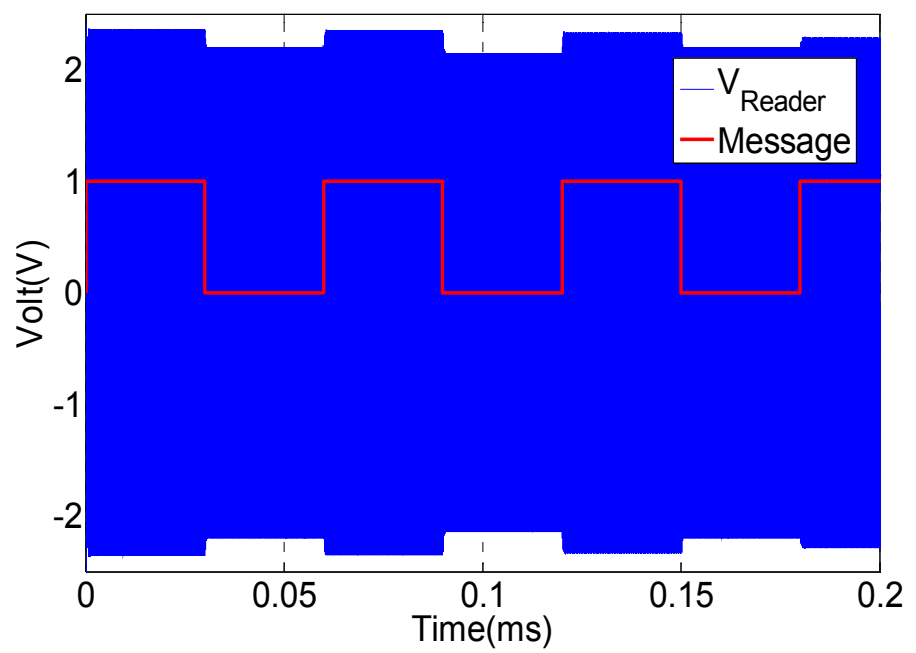

Figure 6.17: Standard SCMR with modulation at the reader.

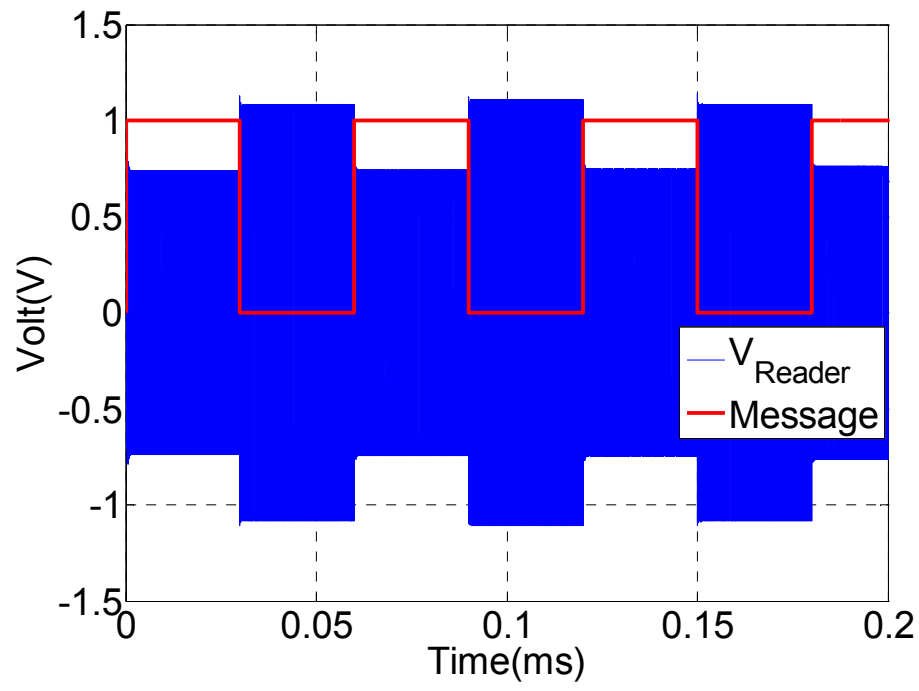

Figure 6.18: Standard SCMR with modulation at the tag. 


\subsubsection{Broadband SCMR}

The result of the broadband SCMR in the proposed design is as shown in Figure 6.19 and Figure 6.20 for the reader and tag respectively. The result shows that modulated backscattering from the tag to the reader occurs.

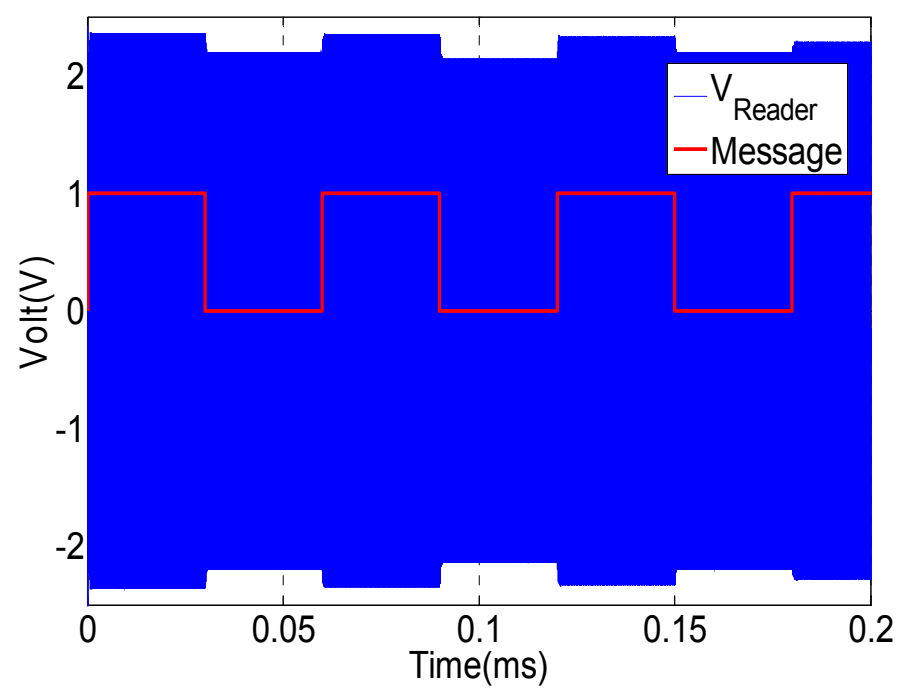

Figure 6.19: Broadband SCMR with modulation at the reader.

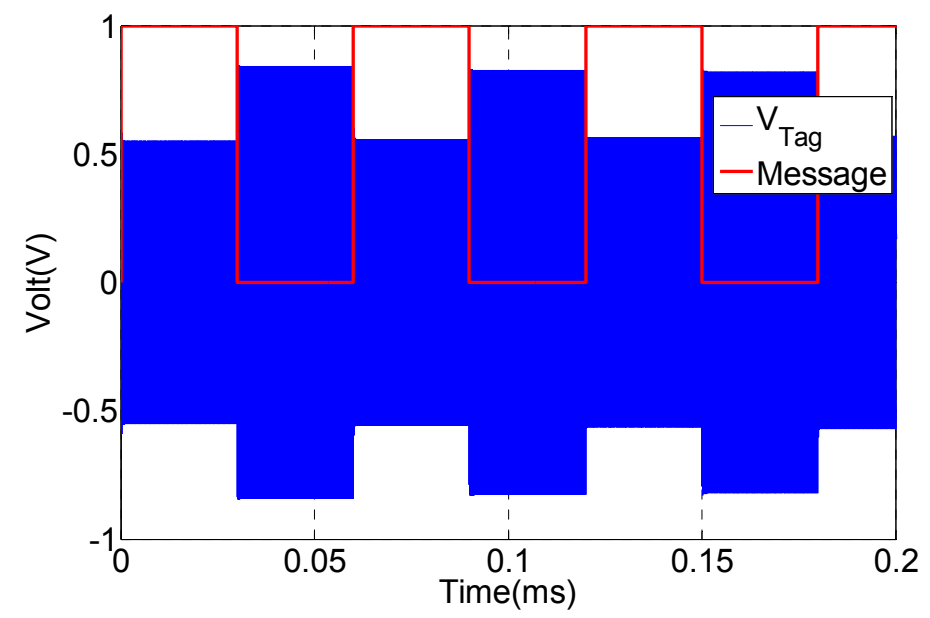

Figure 6.20: Broadband SCMR with modulation at the tag. 


\subsection{Summary}

The results of higher bit rate and bandwidth models show that, broadband SCMR has potential applications in RFID. However, standard SCMR results in over-modulation and modulated backscattering of the message to the reader does not occur. In addition, when the bit rate was reduced and bandwidth requirement reduced to $(15 \mathrm{kbps}) 30 \mathrm{kHz}$, in all three models there were no over-modulation and modulated backscattering of the message to the reader occurs. 


\section{CHAPTER 7}

\section{MISALIGNMENT INSENSITIVE SCMR SYSTEM}

Standard SCMR devices are highly sensitive to misalignment resulting in significant power losses when the orientation of any of the device are changed, the work of several author have highlighted some of the sensitivity issues, however none has shown a process or model that is insensitive in several directions. The three common types of misalignments are axial, lateral and angular misalignments.

A main drawback of standard SCMR systems is that they are highly sensitive to the alignment between transmitter and receiver. An optimization technique for improving the efficiency of SCMR systems under lateral misalignment was presented in [18]. Specifically, 48.4\% efficiency was achieved by using an adaptive matching network. However, [18] did not provide any solution for angular misalignment. Analytical formulations for the power transfer efficiencies of loosely coupled inductive links under lateral and angular coil misalignment were presented in [19]. However, no solutions that address the effects of misalignment were discussed in [19]. The effects of changing the coil separation distance as well as the lateral and angular alignment, between transmitter and receiver in resonant coupling system were also examined. In addition, the ability to transfer power and data were demonstrated via resonant coupling system was reported in [20]. In addition, the effects of misalignment on the efficiency of resonant and inductively coupled RFID systems were examined using analytical formulations and FEM simulation in[21]. 
SCMR's radial and angular misalignment sensitivity were examined by [22] and [23], respectively. Only [23], attempted to correct SCMR's angular misalignment sensitivity by using tuning circuits, which were not able to maintain high efficiency above $60^{\circ}$ of misalignment. In addition, tuning circuits add to the complexity of SCMR RX systems and they cannot compensate for large angular and radial misalignments, as they cannot recover the lost flux density between TX and RX. However, tuning circuits can be useful for compensating the effects of variable axial distance between TX and RX [24], Even though these papers has examined the effects of both lateral and angular misalignment of SCMR systems. However, no solutions have been proposed to address the sensitivity of SCMR systems to lateral, angular and the test for the isotropy of WPT misalignment. Despite the fact that, some of these papers has examined the effects of both lateral and angular misalignment of SCMR systems. However, no solutions have been proposed to address the sensitivity of SCMR systems to lateral, angular and test for isotropy of WPT misalignment.

\subsection{Coil Misalignment Cases}

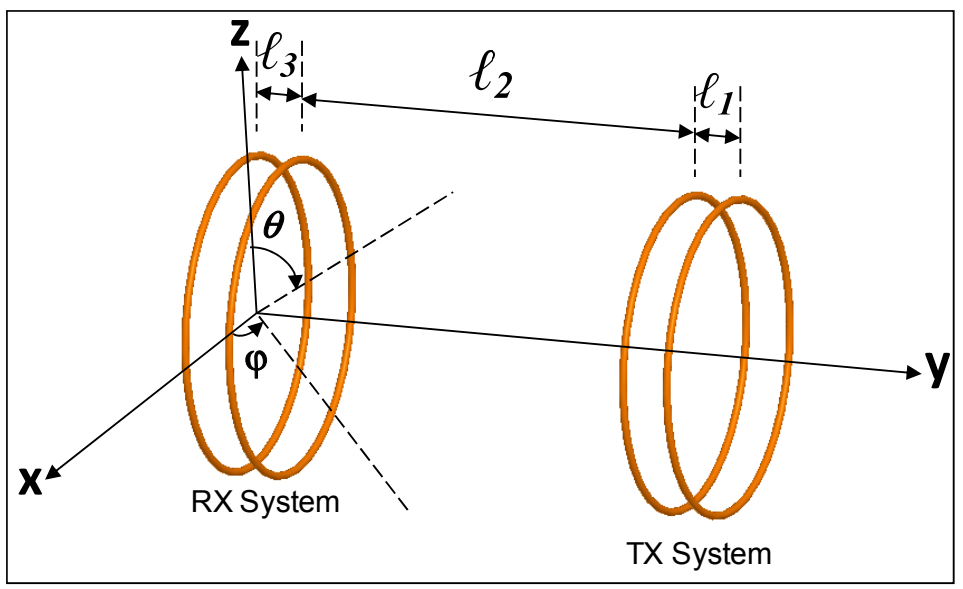

Figure 7.1: SCMR system with angular elevation, $\theta$ and angular azimuth misalignment angles, $\varphi$. 
Coil orientation and alignment are critical in the design of efficient magnetic resonance coupled systems. A conventional SCMR system exhibits high sensitivity to lateral, test for the isotropy of WPT and angular misalignments cases. In Figure 7.1, a simple and typical SCMR system is shown with the angular elevation and angular azimuth misalignments cases. In what follows, we explain the different types of misalignment cases that will be considered here

\subsubsection{Angular elevation misalignment}

In this case, the RX devices (RX resonator and load element) is rotated in the YZ(elevation) plane from $\theta=0^{\circ}$ to $90^{\circ}$ from the center of the two devices on its axis, while the TX devices (TX resonator and source element) are fixed, as shown in Figure 7.1. where $\ell_{1}$ is the distance between source element, and TX element, $\ell_{2}$ is the distance between the TX and RX elements, and $\ell_{3}$ is the distance between the RX element and the load element.

\subsubsection{Angular azimuth misalignment}

In this case, the RX system rotates around its axis in the XY (azimuth) plane from $\varphi=0^{\circ}$ to $360^{\circ}$, while the TX system is fixed shown in Figure 7.1.

\subsubsection{Lateral misalignment}

In this case, the RX system is misaligned by moving it parallel to the TX system and keeping the distance between $\mathrm{RX}$ and TX $\ell_{2}$ constant, while the lateral misalignment offset distance, $D$ increases as shown in Figure 7.2. 


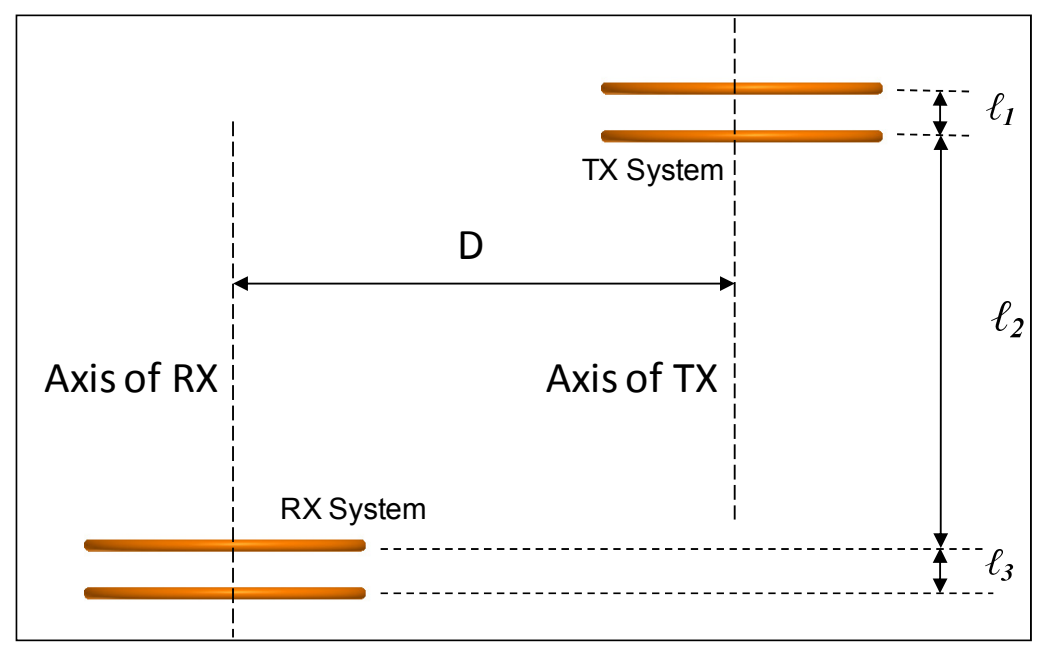

Figure 7.2: SCMR power transfer system with lateral misalignment.

\subsubsection{Test setup for isotropy of WPT}

In this case, the RX device moves around the fixed TX elements and keeping the distance between RX and TX constant. Therefore, the RX elements move by following the trace of a circle in the elevation and azimuth plane, as shown in Figure. 7.3. This test setup is used to examine the wireless powering isotropy of the different SCMR system.

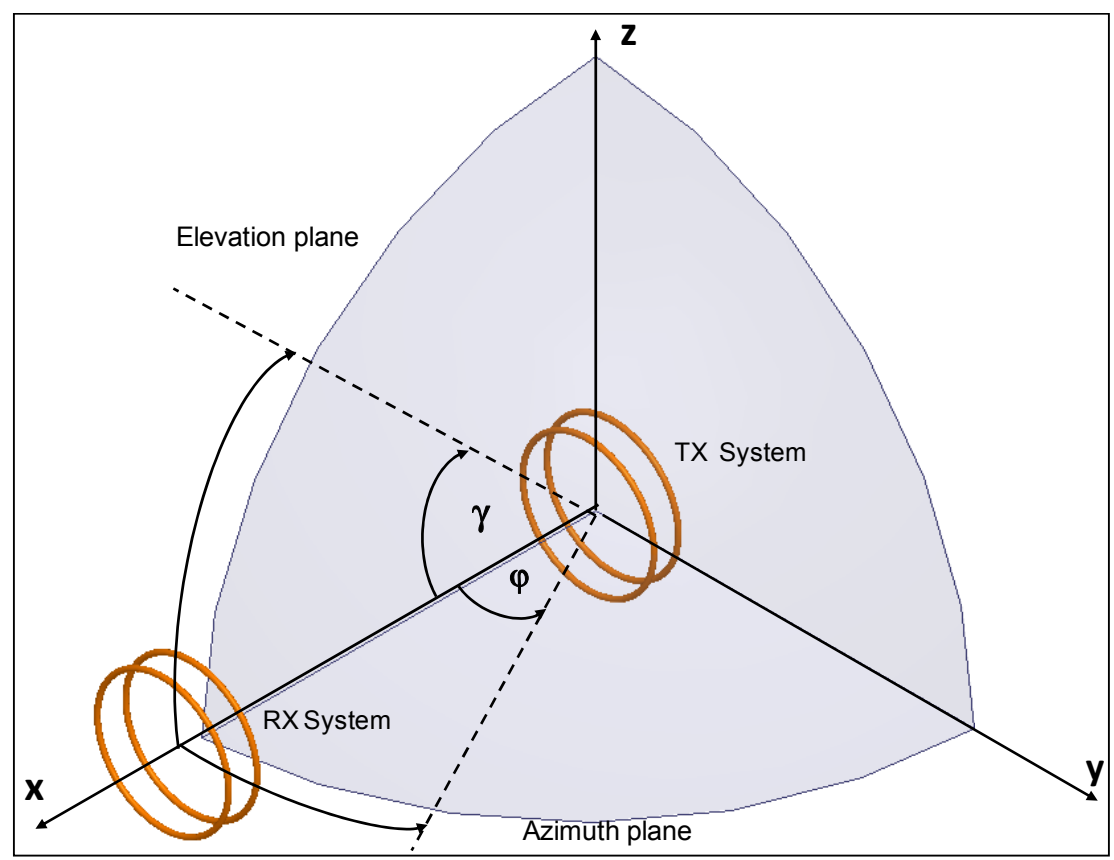

Figure 7.3: SCMR power transfer system with test for isotropy of misalignment angle. 


\subsection{Misalignment analysis}

In this section, the performance of standard SCMR systems will be compared to the performance of novel SCMR system for various misalignment conditions. Standard SCMR systems consist of four elements (see Figure 7.4) and require that TX and RX systems be aligned in order to achieve high efficiency. In fact, such standard SCMR systems suffer significant decrease of their wireless powering efficiency when they are misaligned. The high sensitivity of standard SCMR to misalignment is a major disadvantage for many applications, such as, implantable or wearable devices, biosensors, and other mobile devices, where alignment between TX and RX not only cannot be guaranteed but also it changes due to movement or mobility. Hence, there is a need for new misalignment insensitive and high efficient SCMR designs that can solve this practical problem.

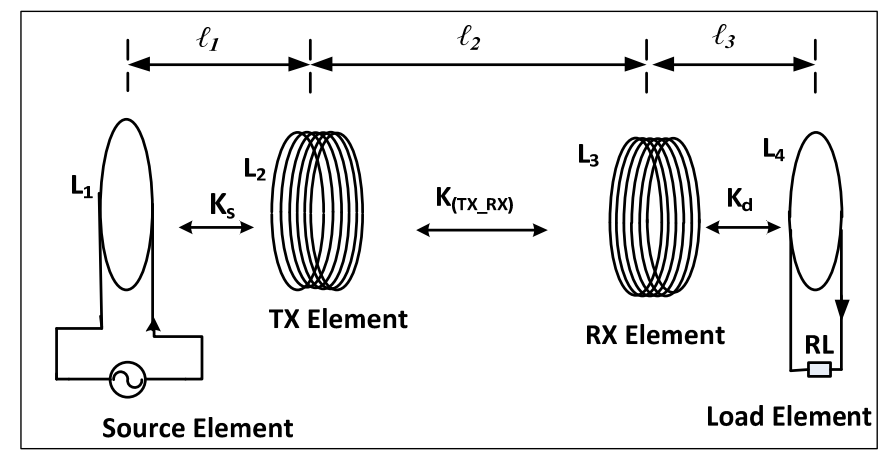

Figure 7.4: Schematic of an SCMR power transfer system in air.

Figure 7.5(a) shows a standard SCMR system, our proposed novel misalignment insensitive SCMR systems are shown in Figure 7.5(b) to Figure 7.5(f). All the simulations in this paper used Ansoft HFSS and Nexxim. The performances of the SCMR systems are shown in Figure 7.5(a) - Figure 7.5(f) are examined for the different misalignment conditions in Section II. 


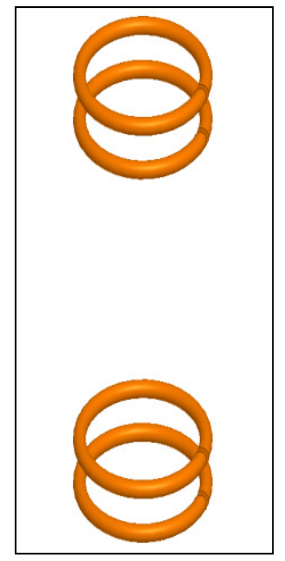

(a)

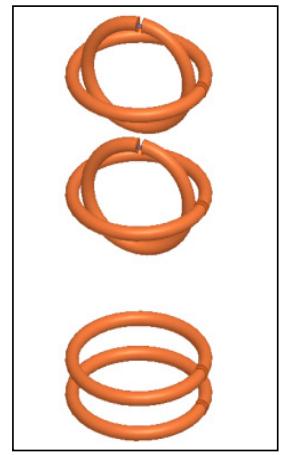

(c)

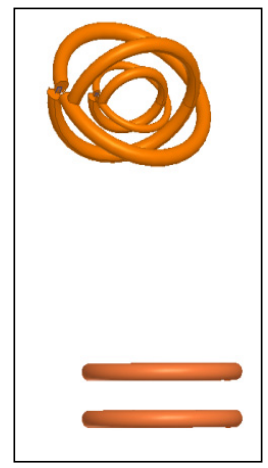

(e)

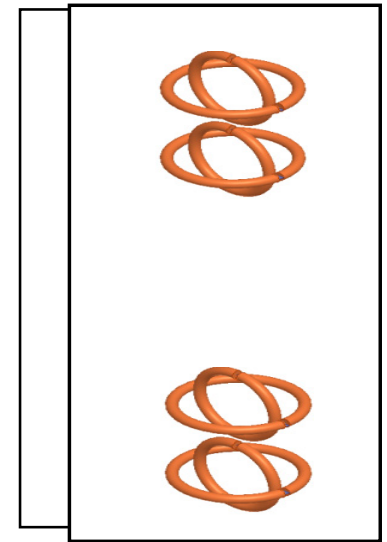

(b)

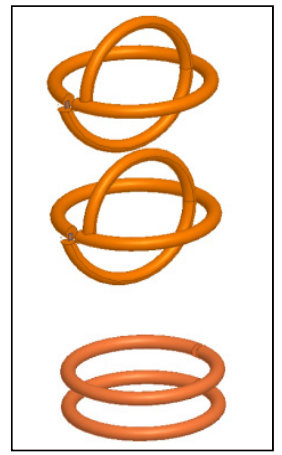

(d)

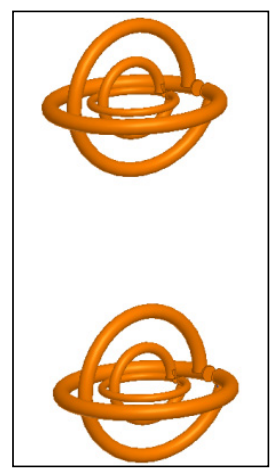

(f)

Figure 7.5. Different SCMR systems. (a) standard SCMR with parallel loops elements, (b) SCMR with orthogonal loop disconnected elements, (c) SCMR with two orthogonal (disconnected) loops and two parallel loop elements, (d) SCMR with two orthogonal (connected) loops and two parallel loop elements, (e) SCMR with two orthogonal embedded (connected) loops and two parallel loop elements (f) misalignment Insensitive SCMR device (2- loop structure). 


\subsection{Standard SCMR}

First, the performance of standard SCMR, which is shown in Figure 7.5(a), is examined under different misalignment conditions. The standard SCMR system was designed for optimal performance following the equations presented in [102]. Figure 7.6 compares the simulation and measurement results of the SCMR system. Specifically, Figure 7.6(a), 7.6(b) and 7.6(c) shows the variation of the SCMR's efficiency for elevation, angular and lateral misalignment conditions respectively.

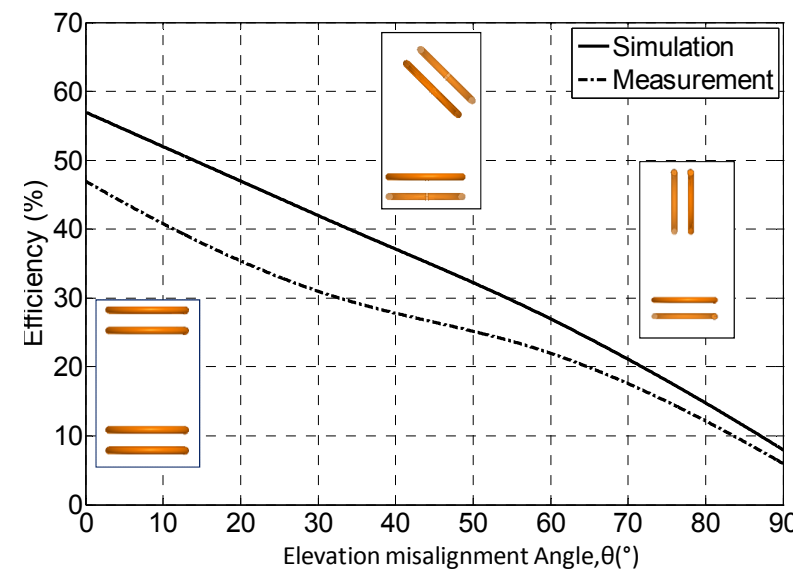

(a)

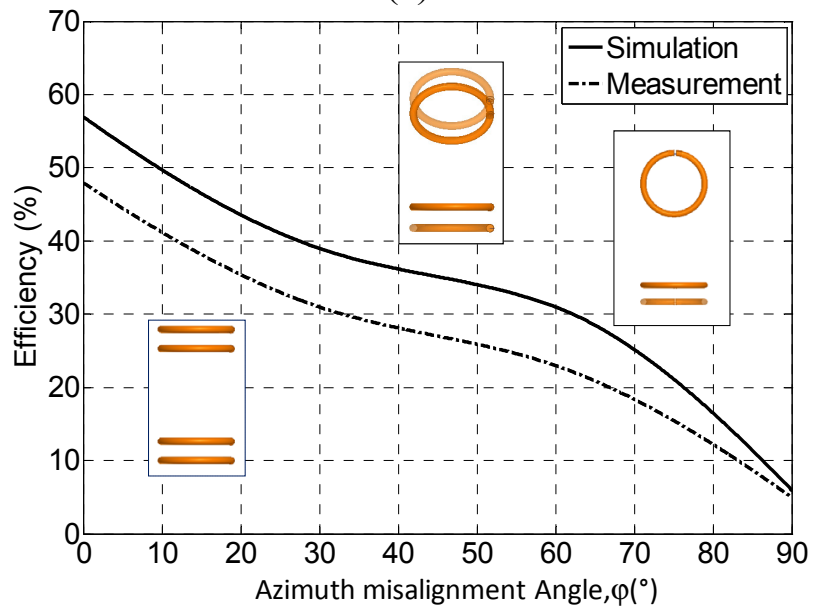

(b) 


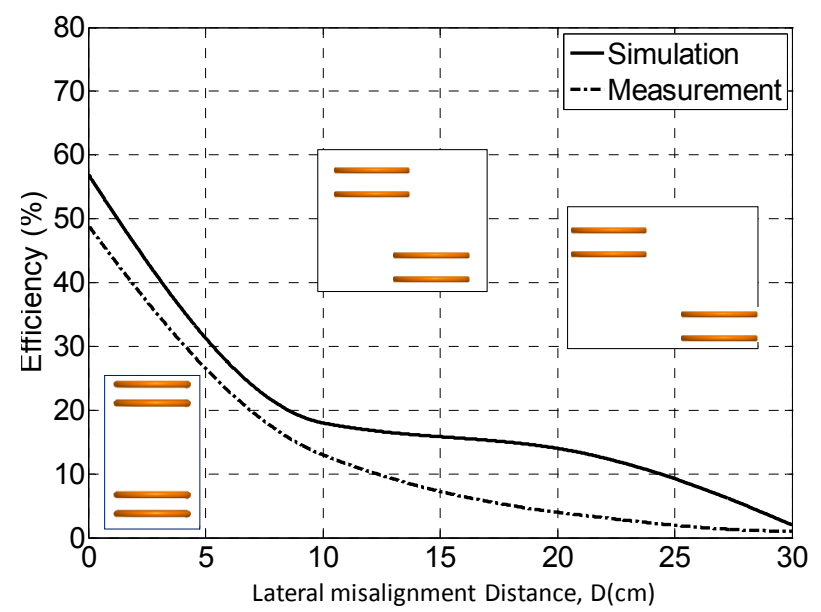

(c)

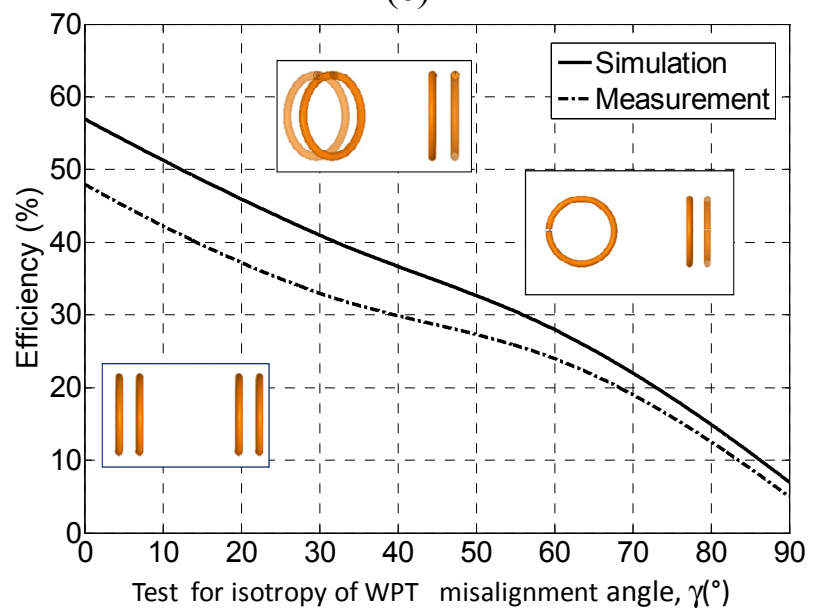

(d)

Figure 7.6: Standard SCMR with the coils different misalignment. (a) Angular elevation, (b) angular azimuth, (c) lateral and (d) test for isotropy of WPT.

The specification of the models in the simulations and measurements are the radius of the loop, $r_{l}=50 \mathrm{~mm}$, the cross sectional wire radius, $r_{c}=2.2 \mathrm{~mm}$, the distance between the TX and RX devices, $D=200 \mathrm{~mm}$, and the capacitor is $68 \mathrm{pF}$. Figure 7.6(a) - Figure 7.6(c) show that as the elevation, azimuth and lateral misalignment increases the efficiency rapidly decreases. These results verify the high sensitivity of conventional SCMR systems to misalignment. Also, the isotropy of the SCMR system was examined using the test setup of Figure 3 and the result is shown in Fig 6 (d), the result also illustrates that the efficiency of the standard SCMR system does not exhibit isotropy. 


\subsection{SCMR with four disconnected orthogonal loop pairs}

In Figure 7.5(b), the standard SCMR system is modified to include four pairs of orthogonal loops (disconnected) to achieve misalignment insensitivity, for the different misalignment angles and cases (see Section II). However, from the simulation result (see Figure 7.7) the misalignment insensitivity was still poor and the power transfer efficiency was worse than standard SCMR, because of the increases in the distance between the adjacent orthogonal loops(TX to source elements and RX to load elements) compared to standard SCMR, resulting in lower coupling coefficient. Measurement analyses were not carried because the simulations indicated that the system would not be efficient.

\subsection{SCMR with two orthogonal and two parallel loops}

In Figure 7.5(c) and Figure 7.5(d), another SCMR system is proposed comprising of is further modified to include pair of orthogonal loops (inter-connected or disconnected) and a pair of parallel loops as seen in Figure 7.5(c) and Figure 7.5(d) respectively, The orthogonal loops are the moving receiver and the parallel loops are the

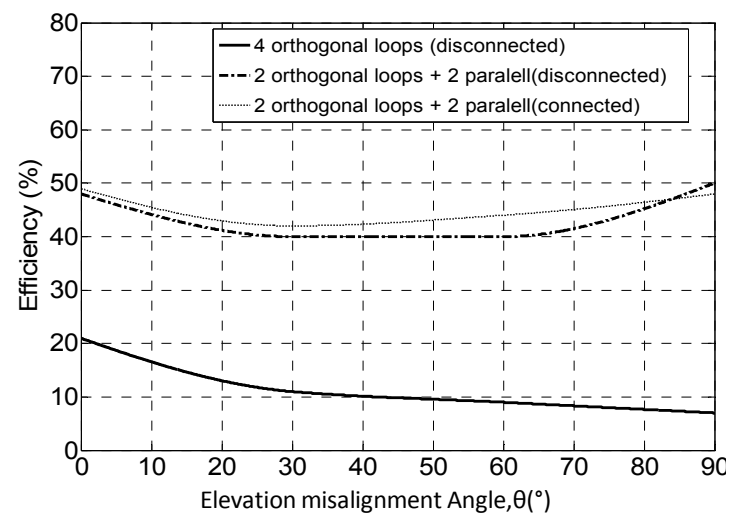

(a) 


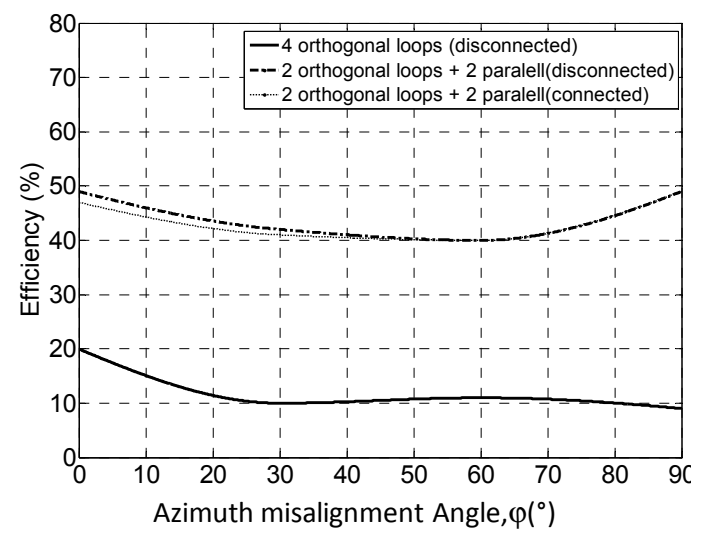

(b)

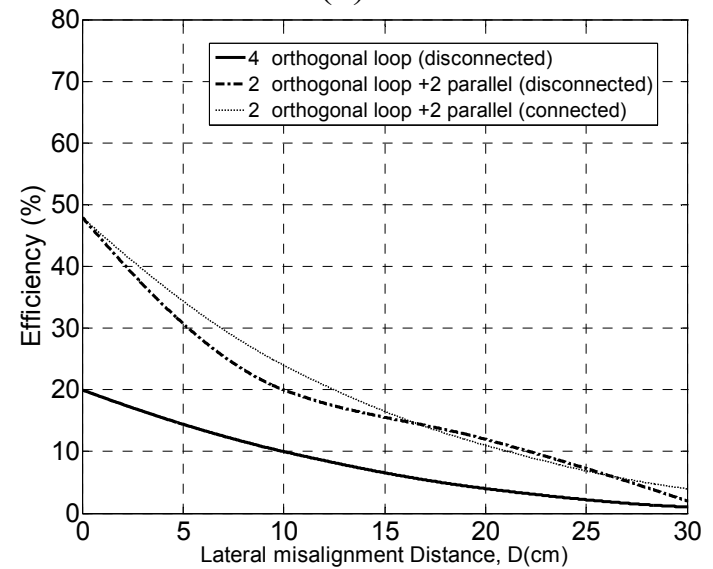

(c)

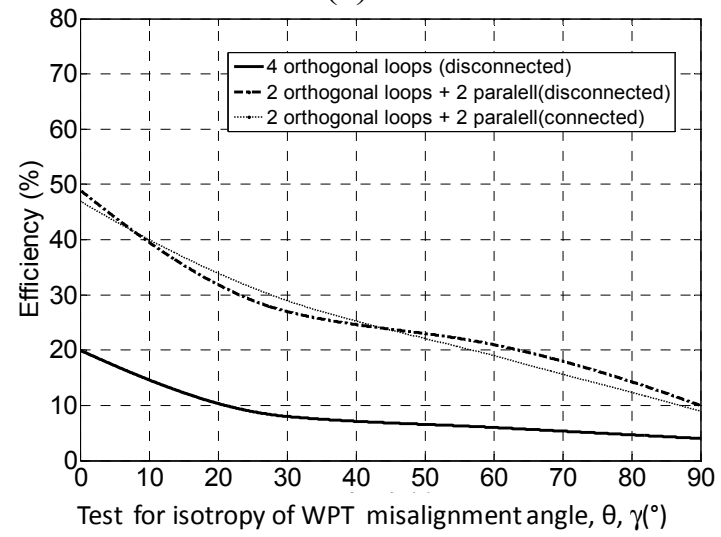

(d)

Figure 7.7: The modified SCMR with misalignment. (a) angular elevation, (b) angular azimuth (c) lateral and (d) test for isotropy of WPT.

fixed transmitter. For the two SCMR systems of Figure 7.5(c) and Figure 7.5(d) for different misalignment conditions (see Figure 7.7), it can be seen that the system exhibit less misalignment sensitivity when compared to standard SCMR. 


\subsection{SCMR with embedded orthogonal loops}

In an effort to further improve the performance of the SCMR system of Figure 7.5(d) we embedded the load element inside the RX resonator (see Figure 7.5(e)), and examined the effects of different misalignment conditions. The simulations and measurement result are shown in Figure 7.8, which illustrates that the system of Figure 7.5(e) has significantly improved misalignment insensitivity compared to standard SCMR. This can be attributed to the high coupling coefficient from the pair of parallel loops (similar to standard SCMR) between the TX resonator and source, and the embedded RX devices which also has increase coupling coefficient and orientation insensitive structure and also because they are embedded. However, the isotropy of this SCMR system was also studied and the result is shown in Fig 7.8 (d), the result shows that the efficiency of the standard SCMR system does not exhibit isotropic behavior. The specification of the models in the simulations and measurements are outermost loop radius, $r_{l}=50 \mathrm{~mm}$, inner loop radius, $r_{2}=45 \mathrm{~mm}$, cross sectional radius, $r_{c}=2.2 \mathrm{~mm}$, distance between TX and RX devices, $D=200 \mathrm{~mm}$, and the capacitors are $56 \mathrm{pF}$ and $68 \mathrm{pF}$ in the orthogonal and the parallel elements.

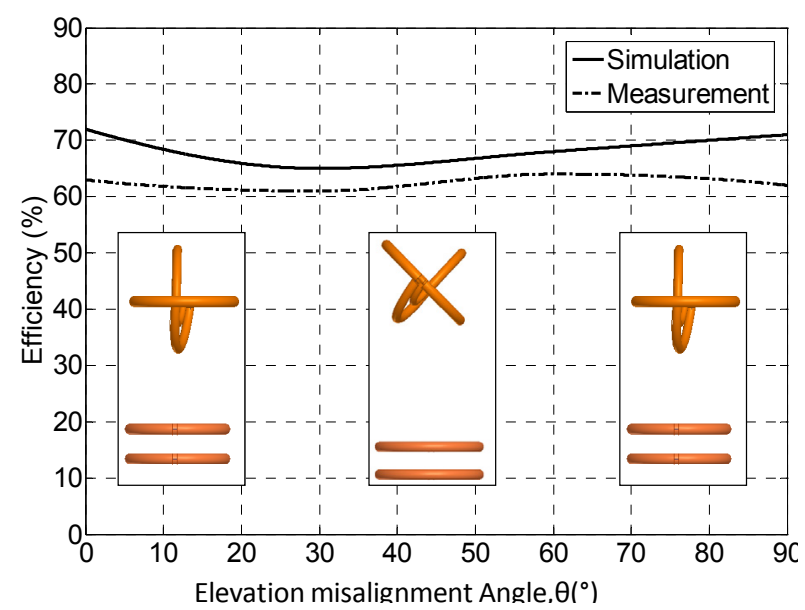

(a) 


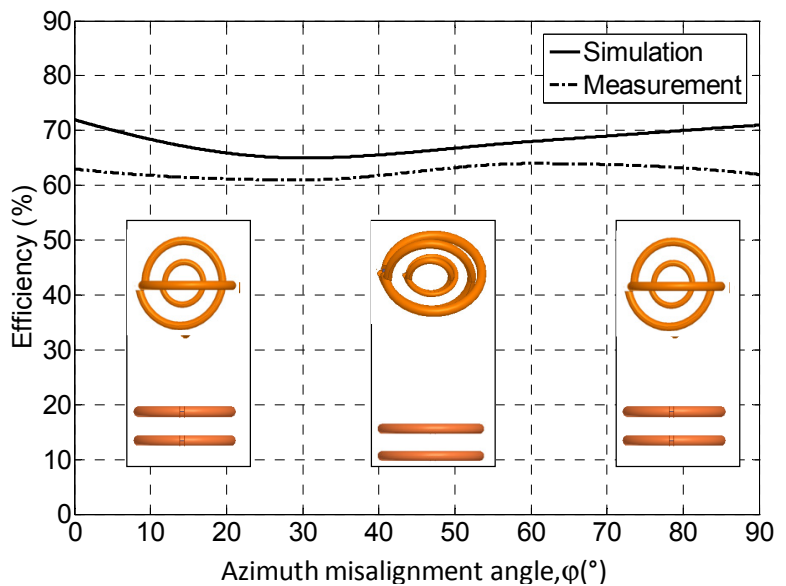

(b)

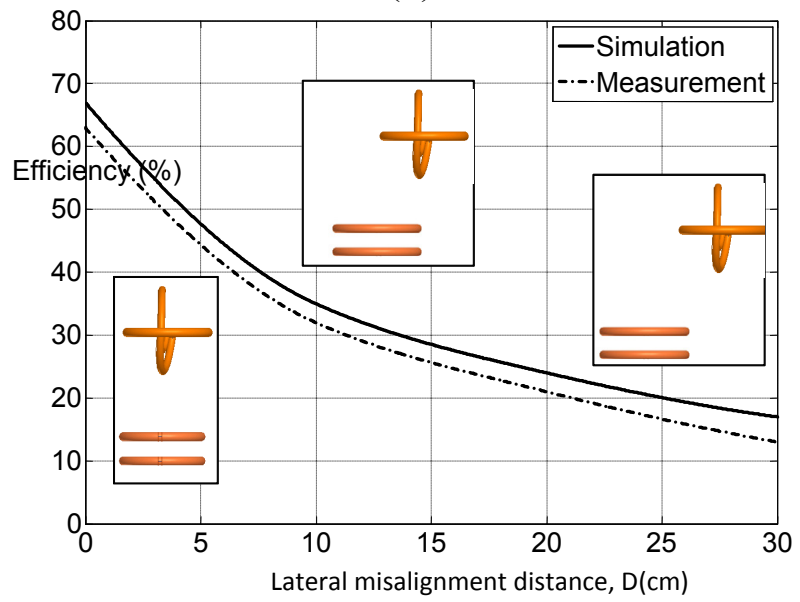

(c)

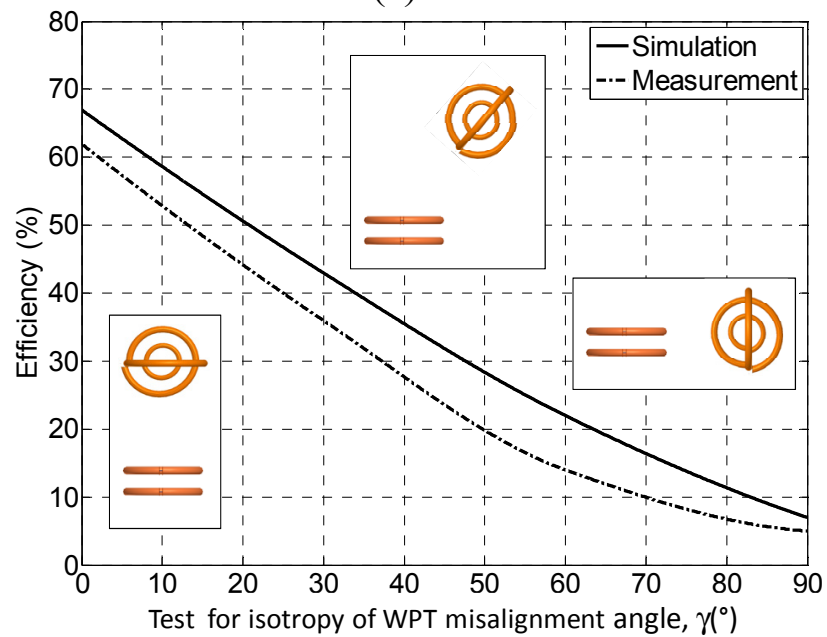

(d)

Figure 7.8: SCMR with two connected orthogonal (embedded) loops and two parallel loops misalignment cases. (a) angular elevation (b) angular azimuth, (c) lateral misalignment, and (d) test for isotropy of WPT. 


\subsection{Misalignment Insensitive SCMR device (2- loop structure)}

A novel SCMR system is proposed here and it is shown in Figure 7.5(f). In this SCMR system, the RX and TX resonator elements as well as the source and load elements are 3-D continuous loops. In addition, the source and load loops are embedded inside the TX and RX resonators respectively. This type of system has a spherical symmetry and thereby expected to have misalignment insensitive performance. Figure 7.9 illustrates the simulation and measurement results of the system for different misalignment conditions. The specification of the models in the simulations and measurements are: the radius of the outermost loop, $r_{1}=50 \mathrm{~mm}$, the radius of the inner loop, $r_{2}=45 \mathrm{~mm}$, the cross sectional wire radius, $r_{c}=2.2 \mathrm{~mm}$, the distance between the TX and RX devices, $D=200 \mathrm{~mm}$, the capacitors are $56 \mathrm{pF}$.

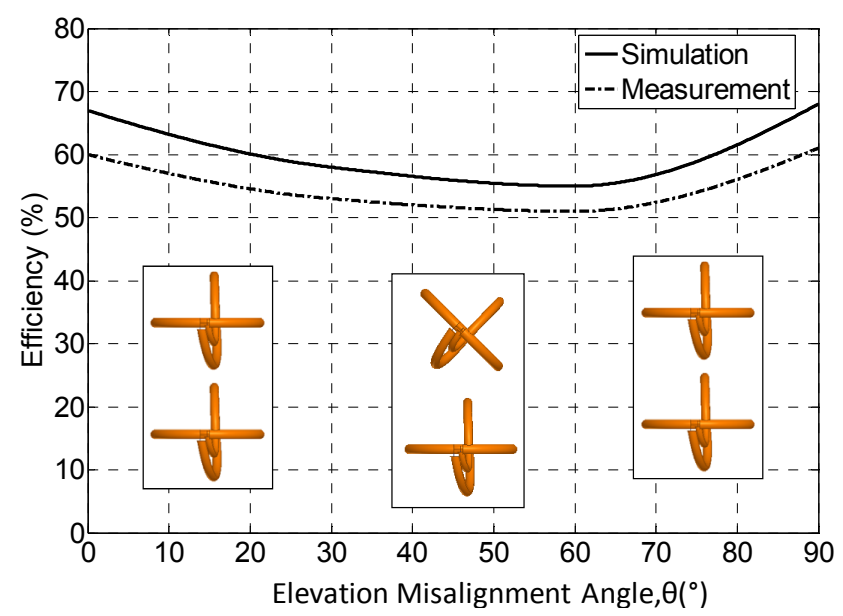

(a) 


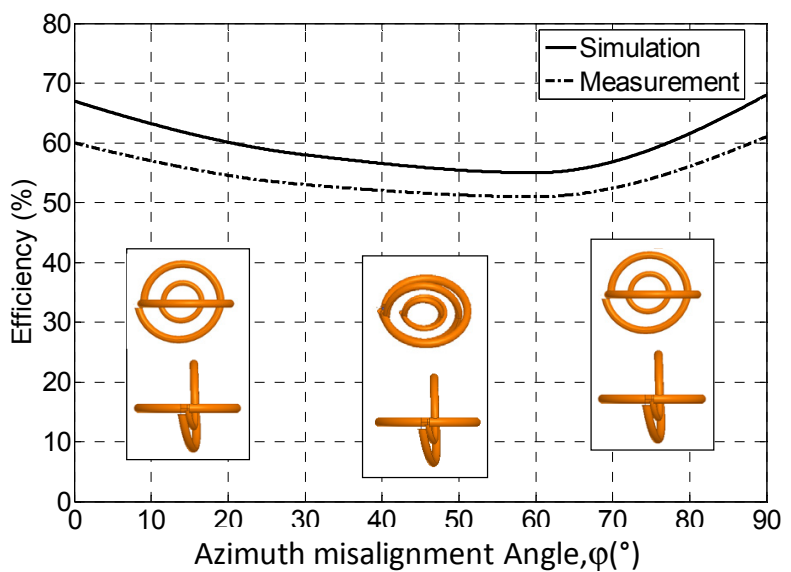

(b)

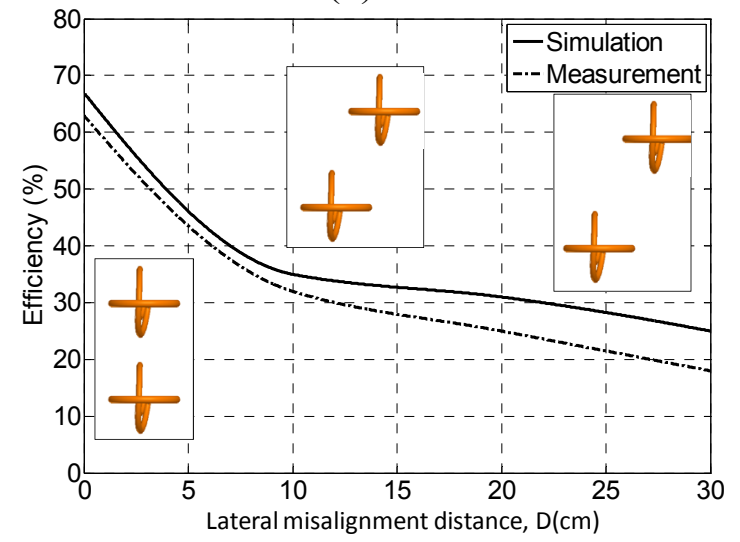

(c)

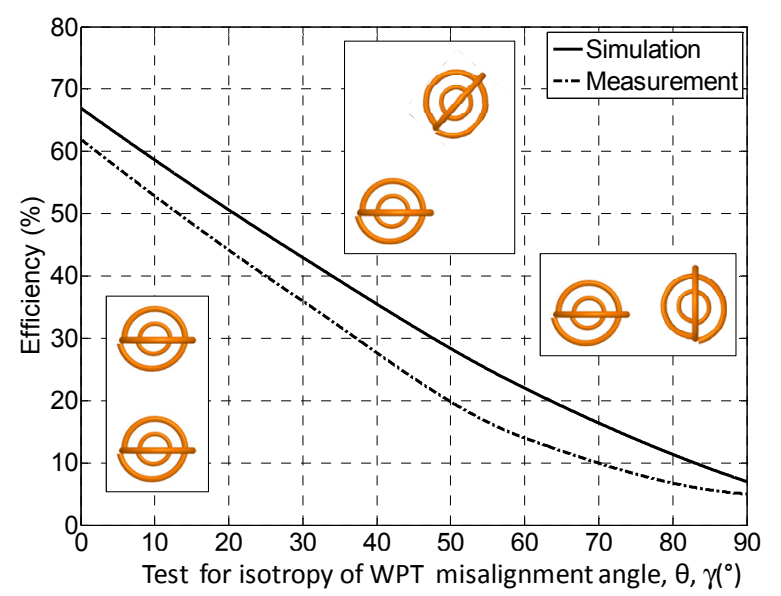

(d)

Figure 7.9: SCMR with four connected orthogonal loops (2-loop structure) misalignment cases. (a) angular elevation, (b) angular azimuth, (c) lateral, and (d) test for isotropy of WPT. 
Figure 7.9(a) and Figure 7.9(b) show that the efficiency of this system is insensitive to angular azimuth and elevation misalignment. In fact, the performance of this system is significantly better than standard SCMR. Fig 7.9(c) shows that the efficiency of the proposed system does not exhibit isotropic performance, which is desirable. In order to mitigate this and develop and SCMR system that is isotropic, further modification of the design is required. The measurement setup for Figure 7.9 is shown in Figure 7.10.

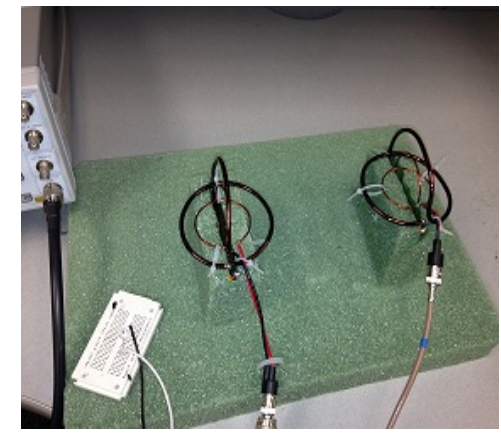

(a)

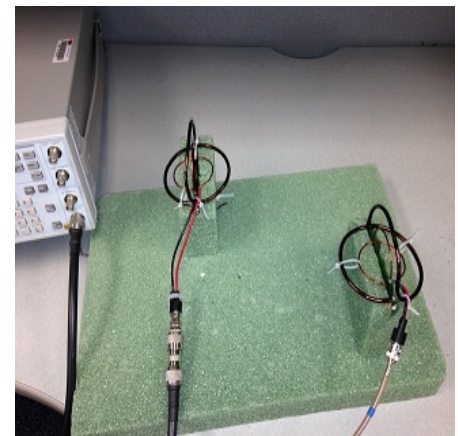

(b)

Figure 7.10: The measurement setup of the misalignment insensitive SCMR (2- loop structure) (a) without misalignment (b) with lateral misalignment.

\subsection{Misalignment Insensitive SCMR (3- loop structure)}

In an effort to develop an SCMR system that is misalignment insensitive and isotropic, we designed the system as shown in Figure 7.12(b). The new system is similar to the design of Figure 7.5(f), also shown in Figure 7.11 (a) for direct comparison with the system of Figure 7.11(b). The difference of the system in Figure 7.11(b) is that each element (TX resonator, RX resonator, source and load) consist of a 3D loop that comprises of three connected orthogonal loops. Figure 7.12 shows that this system will be misalignment insensitive and efficient in these directions, and Figure 12 (d) show that the system is efficient with lateral misalignment. The specification of the models in the 
simulations and measurements are the radius of the outermost loop, $r_{1}=50 \mathrm{~mm}$, the radius of the inner loop, $r_{2}=45 \mathrm{~mm}$, the innermost radius of the loop, $r_{3}=40 \mathrm{~mm}$, the

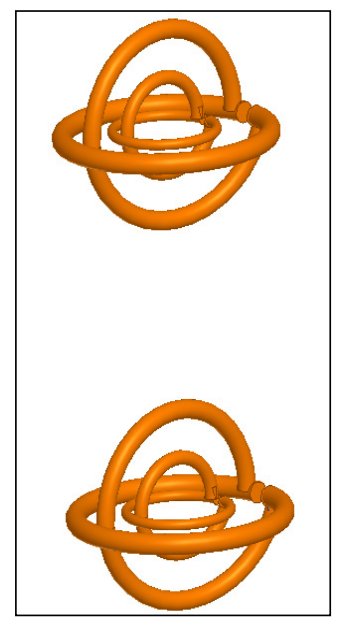

(a)

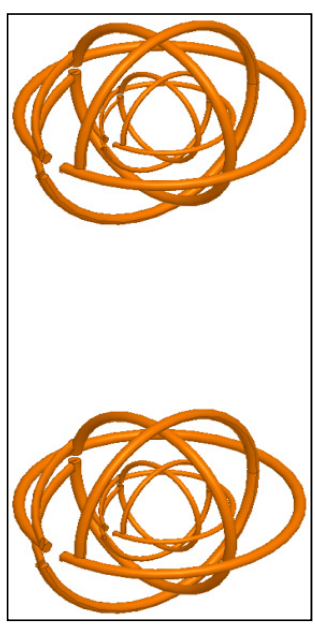

(b)

Figure 7.11: SCMR structures with misalignment insensitivity. (a) SCMR with two orthogonal loops each (connected) and embedded. (b) SCMR with three orthogonal loops each (connected) and embedded.

cross sectional wire radius, $r_{c}=2.2 \mathrm{~mm}$, the distance between the TX and RX devices, $D=200 \mathrm{~mm}$, resonance frequency, $f_{r}=41.5 \mathrm{MHz}$. The capacitor of the 3-loop SCMR system is $22 \mathrm{pF}$. The measurement setup for Figure 7.12 is shown in Figure 7.13. The challenges of implementing this system can be solved by embedding the elements in the body of the mobile device of interest. The system efficiency and range can be further improved by working with other materials and geometries.

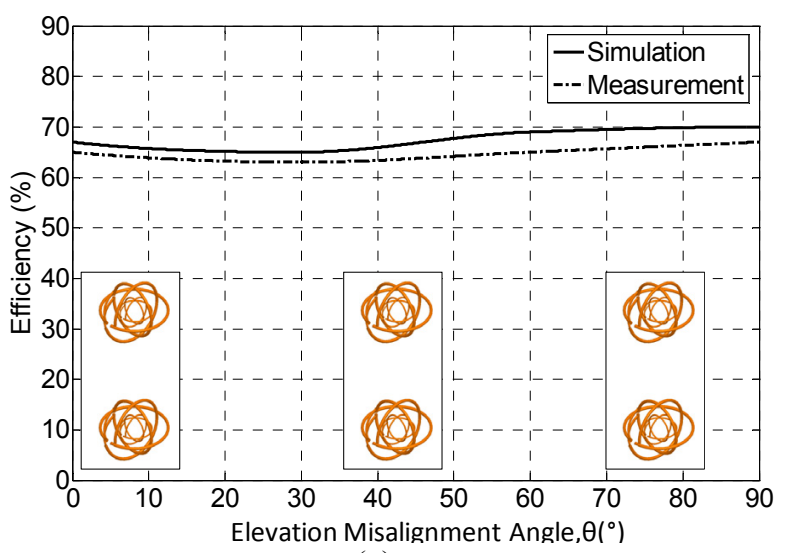

(a) 


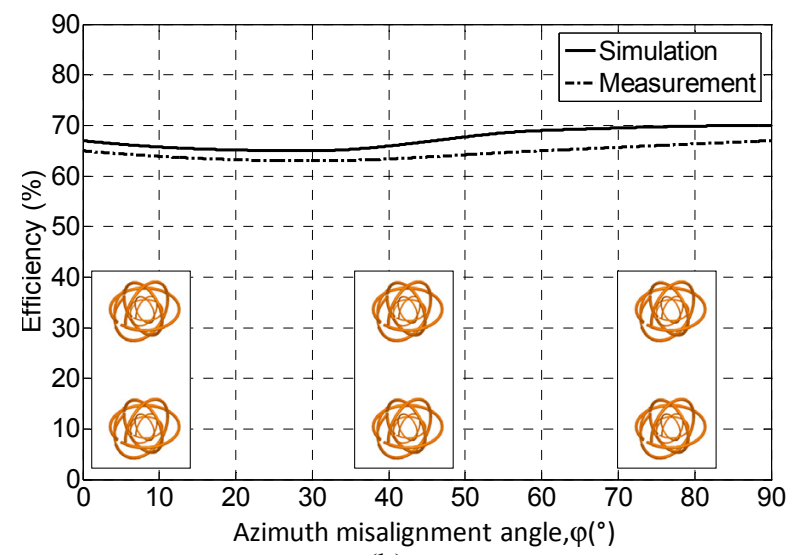

(b)

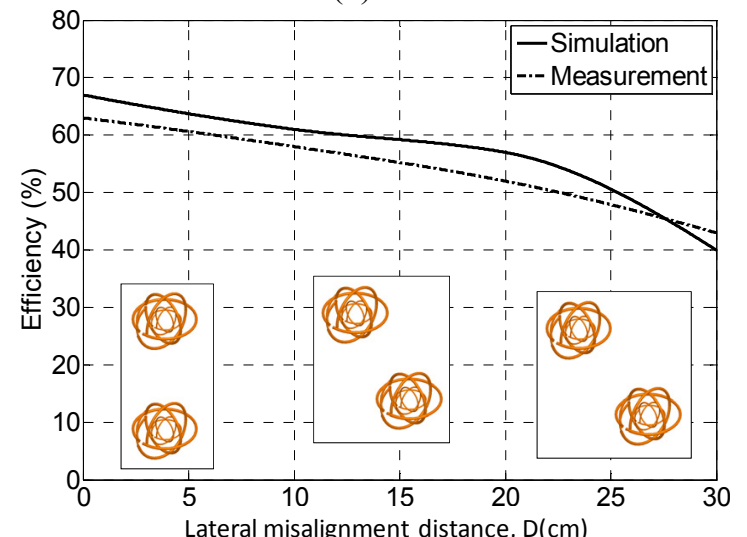

(c)

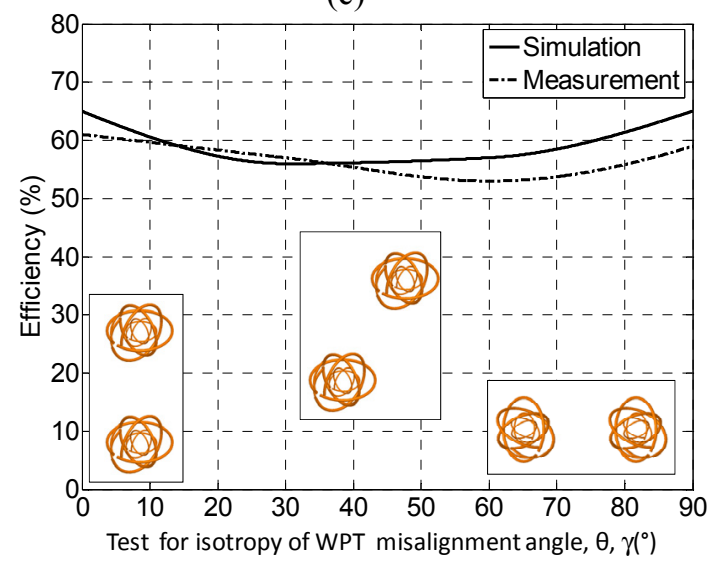

(d)

Figure 7.12: SCMR with orthogonal (connected) loops, the source and load are embedded in the TX and RX devices (3-loop structure) with misalignment cases. (a) angular elevation, (b) angular azimuth, (c) lateral and (d) test for isotropy. 


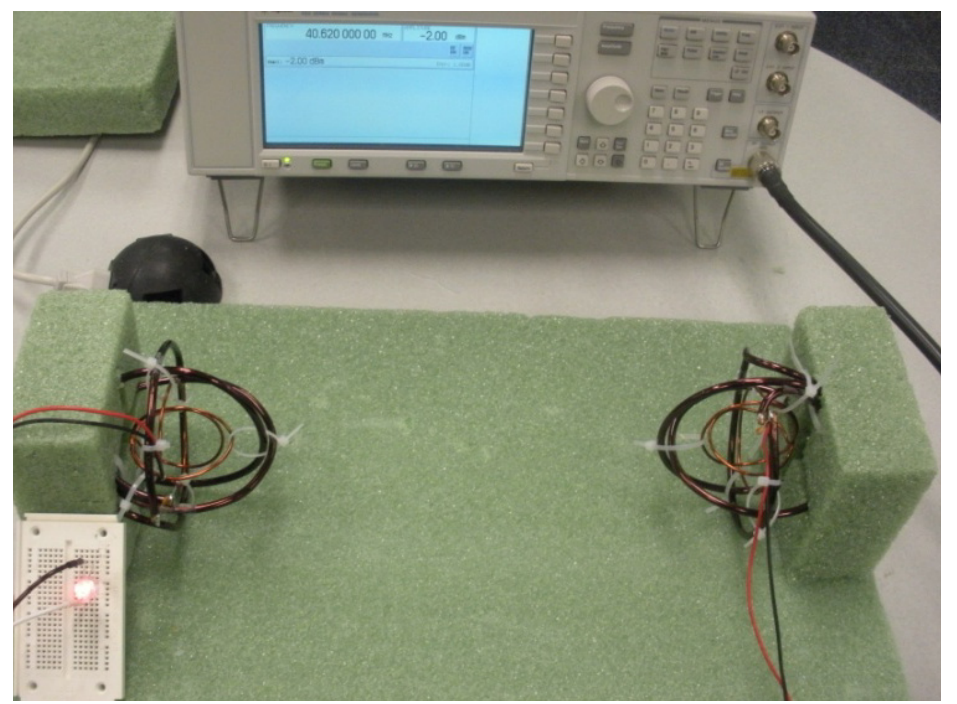

Figure 7.13: shows the measurement setup for the misalignment insensitive SCMR (3loop structure).

\subsection{Summary}

The work presented various optimized designs of SCMR elements, 2-loops and 3loops structures with misalignment-insensitive performance. Five setups were proposed, of connected or disconnected loops, which are either parallel, orthogonal, embedded or a combination. The optimal SCMR features power transfer efficiencies above $50 \%$ over the complete misalignment range of $0^{\circ}-90^{\circ}$ degrees in a performance dramatically better than typical SCMR elements utilized for wireless power transfer applications that suffer of efficiencies down to $10 \%$ in extreme misalignment topologies. 


\section{CHAPTER 8}

\section{CONCLUSIONS AND FUTURE WORK}

\subsection{Conclusions}

This dissertation presented a detailed analytical optimization of SCMR elements with loops, helices and spiral structures. In the loop and helices cases, our formulations proved that the global maximum Q-factor of a loop or helix is achieved when the ratio between the radius, $r$, and the cross-sectional radius, $r_{c}$, of the loop is approximately equal to 9.52 . This important finding can be used to design maximally efficient SCMR wireless powering systems that use loops or helices as transmitting and receiving elements. In spiral, the optimization numerically proved that the global maximum Qfactor of a spiral exist and the optimal design of SCMR systems that use spiral resonators can be achieved.

Using the optimal parameters derived, wireless power transfer to sensors embedded in plain and reinforced concrete structures for SHM applications via SCMR were investigated with loops, helices and spiral structures. The extended Debye model was also used to describe the electromagnetic properties of concrete. The result shows that maximum efficiencies that range from $27 \%$ at $12 \%$ humidity to $59.0 \%$ at $0.2 \%$ humidity inside concrete at a depth of $10 \mathrm{~cm}$. It also shows that SCMR can transfer power more efficiently than other methods and the loss are closely related to the humidity conditions of concrete and depth.

Furthermore, the use of the optimal SCMR device to power Wearable and Implantable Medical Devices (WIMD) were also investigated. The result showed that 
WIMD can based on SCMR can be used to efficiently power biomedical implants, the high efficiency enables the SCMR device to deliver a significant amount of power to biomedical implants without excessive transmitter compared to resonant coupling in terms of the Specific absorption rate (SAR) levels for the same power delivered. The result also shows that, the SCMR has great potentials in WMD, and has very low SAR, electric and magnetic field distribution close to the tissue, which is important for biomedical applications. It is a planner, hence can be embedded in clothing easily.

The Standard SCMR structures are highly sensitive to misalignment and significant power losses when the orientation of any of the devices is changed, this work also presented various optimized designs of SCMR elements, using 2-loops and 3-loops structures with misalignment-insensitive performance. The optimal SCMR exhibits high power transfer efficiencies above $50 \%$ over the complete misalignment range of $0^{\circ}-90^{\circ}$ degrees in several directions and perform dramatically better than typical SCMR elements utilized for wireless power transfer applications that suffer of efficiencies down to less than $10 \%$ in extreme misalignment topologies.

In conclusion, the wireless power transmission systems that we developed showed greater efficiencies than existing solutions, for the plain and reinforced concrete applications and in biomedical for Wearable and Implantable Medical Devices. 


\subsection{Future Work}

This research was centered on optimization of wireless power transfer to sensors embedded in concrete for SHM and WIMD in biomedical applications. However, there are some areas that requires further work.

First part of the work focused on analytical optimization of SCMR elements with loops, helices and spiral structures. More work can be done on miniaturization of the structures for non-homogenous interface (concrete, tissues, etc.), this can be done with a combination of different dielectrics and ferromagnetic materials with very high permeability, to achieve optimal miniaturized embedded structures with high efficiency. This research can be extended to other applications such as water and soil.

In addition, the SCMR is a type of inductive coupling method, and only operated at the reactive near field region, hence it has a limited range. The range can be improved by operating at a lower frequency (therefore increasing the wavelength) and designing the SCMR devices to have structures with special topologies and materials, which might include some types of metamaterials.

Furthermore, part of this work is to maximize the power transfer efficiency to embedded sensors and minimize the required battery charging time. The research can be extended to the optimization of embedded sensors that are completely passive and batteryless.

Finally, part of the SCMR system developed in this work is the multiband SCMR, with two or more frequencies of operation for simultaneous multiple frequencies efficient WPT. This can be improved developing optimal reconfigurable SCMR system to achieve more multiple frequencies WPT and also for directional WPT through beamforming. 


\section{REFERENCES}

[1] S. D. Glaser, H. Li, M. L. Wang, J. Ou, and J. Lynch, "Sensor Technology Innovation for the Advancement of Structural Health Monitoring: A Strategic Program of US-China Research for the Next Decade, " Smart Structures and Systems, vol. 3, no. 2, pp. 221-244, 2007.

[2] J. T. Bernhard, K. Hietpas, E. George, D. Kuchma, and H. Reis, "An Interdisciplinary Effort to Develop a Wireless Embedded Sensor System to Monitor and Assess Corrosion in the Tendons of Prestressed Concrete Girders, " IEEE Topical Conference on Wireless Communication Technology, pp.241-243, 2003.

[3] P.V Nikitin, K.V.S Rao, S. Lazar , "An Overview of Near Field UHF RFID," Proc. RFID IEEE Int. Conf., March 2007, pp. 167 - 174.

[4] K. Fotopoulou, B.W. Flynn, "Optimum antenna coil structure for inductive powering of passive rfid tags," 2007 IEEE International Conference on RFID, vol, no, pp.71,77, 26-28 March 2007.

[5] M. Budhia, G.A. Covic, J.T. Boys, "Design and Optimization of Circular Magnetic Structures for Lumped Inductive Power Transfer Systems," IEEE Transactions on Power Electronics, vol.26, no.11, pp.3096-3108, Nov. 2011.

[6] Ying $\mathrm{Wu}$; Luguang Yan; Shangang $\mathrm{Xu}$, "A new contactless power delivery system," ICEMS 2003. Sixth International Conference on Electrical Machines and Systems, 2003., vol.1, no., pp.253-256 vol.1, 9-11 Nov. 2003.

[7] D. E. Hughes, "Hughes' Research in Wireless Telegraphy", The Electrician, Volume 43, 1899, page 41.

[8] W. C. Brown, "Experiments in the transportation of energy by microwave beam", IEEE Int. Conv. Rec., vol. 12, pp.8-17 1964.

[9] G. D. Arndt and E. M. Kerwin, "Applications of low-earth-orbit power transmission," Space Power, vol. 6, no. 2, pp. 137-155, 1986. 
[10] A. Kurs, A. Karalis, R. Moffatt, J. D. Joannopoulos, P. Fisher, and M. Soljacic, "Wireless Power Transfer via Strong Coupled Magnetic Resonances," Science, Vol. 317, No.5834, pp. 83-86, Jul. 2007.

[11] A. Karalis, J.D. Joannopoulos, and M. Soljacic, "Efficient wireless non-radiative mid-range energy transfer," Elsevier, Annals of Physics, vol. 323, pp. 34-48, January 2008.

[12] A. Kurs, A. Karalis, R. Moffatt and M. Soljacic Marin, "simultaneous midrange power transfer to multiple devices", Applied Physics Letter, vol. 96, 044102, 2010 .

[13] B. L. Cannon, J. F. Hoburg, D.D. Stancil, S.C. Goldstein, "Magnetic Resonant Coupling As a Potential Means for Wireless Power Transfer to Multiple Small Receivers," IEEE Transactions on Power Electronics, vol. 24, no.7, pp.18191825 , July 2009.

[14] C. Rathge; D. Kuschner,; , "High efficient inductive energy and data transmission system with special coil geometry," Power Electronics and Applications, 2009. EPE '09. 13th European Conference on, vol., no., pp.1-8, 8-10 Sept. 2009.

[15] Koh Kim Ean, Beh Teck Chuan, T. Imura, Y. Hori, "Novel band-pass filter model for multi-receiver wireless power transfer via magnetic resonance coupling and power division," 2012 IEEE 13th Annual Wireless and Microwave Technology Conference (WAMICON), vol., no., pp.1-6, 15-17 April 2012.

[16] Lee Jaechun and Nam Sangwook, "Fundamental Aspects of Near-Field Coupling Small Antennas for Wireless Power Transfer," IEEE Transactions on Antennas and Propagation, vol.58, no.11, pp.3442-3449, Nov. 2010.

[17] Lee Seung-Hwan and R.D. Lorenz, "A design methodology for multi-kW, large air-gap, MHz frequency, wireless power transfer systems," 2011 IEEE Energy Conversion Congress and Exposition (ECCE),vol., no., pp.3503-3510, 17-22 Sept. 2011. 
[18] S. G. Lee, H. Hoang, Y. H. Choi, F. Bien, "Efficiency improvement for magnetic resonance based wireless power transfer with axial-misalignment," Electronics Letters, vol.48, no.6, pp.339-340, March 152012.

[19] K. Fotopoulou, B. W. Flynn, "Wireless Power Transfer in Loosely Coupled Links: Coil Misalignment Model," Magnetics, IEEE Transactions on Magnetics, vol.47, no.2, pp.416-430, Feb. 2011.

[20] M. A. Adeeb, A. B. Islam, M. R. Haider, F. S. Tulip, M. N. Ericson, and S. K. Islam, "An Inductive Link-Based Wireless Power Transfer System for Biomedical Applications," Active and Passive Electronic Components, vol. 2012, Article ID 879294, 11 pages, 2012.

[21] Wang Junhua, S. L Ho, W. N Fu and Sun Mingui, "Analytical Design Study of a Novel Witricity Charger With Lateral and Angular Misalignments for Efficient Wireless Energy Transmission," IEEE Transactions on Magnetics, vol.47, no.10, pp.2616-2619, Oct. 2011.

[22] Fei Zhang, S. A Hackwoth, Xiaoyu Liu, Li Chengliu, Sun Mingui, "Wireless power delivery for wearable sensors and implants in Body Sensor Networks," 2010 Annual International Conference of the IEEE Engineering in Medicine and Biology Society (EMBC), vol., no., pp.692-695, Aug. 31 2010-Sept. 42010.

[23] A. P Sample, D. A. Meyer, J. R. Smith, "Analysis, Experimental Results, and Range Adaptation of Magnetically Coupled Resonators for Wireless Power Transfer," IEEE Transactions on Industrial Electronics, vol.58, no.2, pp.544-554, Feb. 2011.

[24] Chen Qiang, K. Ozawa, Yuan Qiaowei, K. Sawaya, "Antenna Characterization for Wireless Power-Transmission System Using Near-Field Coupling," IEEE Antennas and Propagation Magazine, , vol.54, no.4, pp.108,116, Aug. 2012.

[25] C. R. Farrar and K. Worden, "An Introduction to Structural Health Monitoring," Philosophical Transactions of the Royal Society, vol. 365, pp. 303-315, 2007. 
[26] D. Chakraborty, N. Kovvali, J.J. Zhang, A. Papandreou-Suppappola, A. Chattopadhyay, "Adaptive learning for damage classification in structural health monitoring," 2009 Conference Record of the Forty-Third Asilomar Conference on Signals, Systems and Computers, vol., no., pp.1678-1682, 1-4 Nov. 2009.

[27] M. Reyer, S. Hurlebaus, J. Mander, O.E. Ozbulut, "Design of a wireless sensor network for Structural Health Monitoring of bridges," Sensing Technology (ICST), 2011 Fifth International Conference on, vol., no., pp.515-520, Nov. 28 2011-Dec. 12011.

[28] P. Klinkhachorn, A. S. Mercer, U. B. Halabe, and H. G. Rao, "An Autonomous Unmanned Ground vehicle for Non-Destructive Testing of Fiber-Reinforced Polymer Bridge Decks," IEEE Instrumentation \& Measurement Magazine, pp. 28-33, Jun. 2007.

[29] H. Song and V. Sarawathy, "Corrosion Monitoring of Reinforced Concrete Structures- A Review," International Journal of Electrochemical Science, no. 2, pp. 1-28, 2007.

[30] F. Bastianini, F. Matta, A. Rizzo, N. Galati, and A. Nanni, "Overview of Recent Bridge Monitoring Applications using Distributed Brillouin Fiber Optic Sensors", The E-Journal \& Exhibition of Nondestructive Testing, pp. 269-276, 2007.

[31] Structural Health Monitoring Activities at Los Alamos National Laboratory: http://www.cosmos-eq.org/events/Dam_WS/sections/2001_01_3_3sohn.pdf.

[32] P. A 9-Step Process for Developing a Structural Health Monitoring System: http://www.structuremonitoring.com/Graves-Rens-Rutz_SHMprocess 20110502f.pdf

[33] H. Sohn, J. Czarnecki and C. Farrar, "Structural Health Monitoring Using Statistical Process Control", Journal of Strucntural Engineering, pp. 1356-1363, 2000 . 
[34] H. Sohn and C. R. Farrar, "Statistical Process Control and Projection Techniques for Damage Detection," European COST F3 Conference on System Identification and Structural Health Monitoring, pp.105-114, 2000.

[35] G. Naldi and P. Venini, "Postprocessing Singular Solutions by the Wavelet Transform," Structural Damage Assessment Using Advanced Signal Processing Procedures, Proceedings of DAMAS ‘97, pp. 109-120, 1997.

[36] C. Biemans, W. J. Staszewski, C. Boller, and G. R. Tomlinson, "Crack Detection in Metallic Structures Using Piezoceramic Sensors," Damage Assessment of Structures, Proceedings of the International Conference on Damage Assessment of Structures, pp. 112-121, 1999.

[37] H. Sohn, C. R. Farrar, R. M. Hemez, D. D. Shunk, D. W. Stinemates, B. R. Nadler, and J. J. Czarnecki, "A Review of Structural Health Monitoring Literaure: 1996-2001," Los Alamos National Laboratory Report, 2004.

[38] C. H. Loh and C. C. Huang, "Damage Identification of Multi-Story Steel Frames Using Neural Networks," Structural Health Monitoring, pp. 390-399, 2000 .

[39] M. Krawczuk, W. Ostachowicz, and G. Kawiecki, "Detection of Delamination in Cantilevered Beams Using Soft Computing Methods," European COST F3 Conference on System Identification and Structural Health Monitoring, Madrid, Spain, pp. 243-252, 2000.

[40] K. Worden and A. J. Lane, "Damage Identification Using Support Vector Machines," Smart Materials and Structures, vol. 10, pp. 540-547, 2001.

[41] L. L. Lapin, "Probability and Statistics for Modern Engineering," PWS-Kent Publishing, 2nd Edition, 1990.

[42] Y. Q. Ni, K. Y. Wong, and Y. Xia, "Health Checks through Landmark Bridges to Sky-high Structures," Advances in Structural Engineering, vol. 14, no. 1, pp. 103-119, 2011. 
[43] A. Gastineau, T. Johnson, and A. Schultz, "Bridge Health Monitoring and Inspections-A Survey of Methods," Report MN/RC 2009-29, 2009.

[44] S. Kim, "Wireless Sensor Networks for Structural Health Monitoring," Research project, 2005.

[45] F. Akyildiz, W. Su, Y. Sankarasubramaniam, and E. Cayirci, "Wireless Sensor Networks: A Survey," Computer Networks, vol. 38, pp. 393-422, 2002.

[46] V. Snayder, B. Chen, K. Lorincz, T. Jones, and M. Welsh, "Sensor Networks for Medical Care," Technical report, Harvard University, 2005.

[47] J. Anderson, R. Szewczyk, A. Mianwaring, D. Culler, and J. Anderson, "Analysis of Wireless Sensor Networks for Habitat Monitoring," Wireless Sensor Networks, 2004.

[48] D. J. Cook and S. K. Das, "How Smart are Our Environments? An Updated Look at the State of the Art," Pervasive and Mobile Computing, vol. 3, no. 2, pp. 53-73, 2007.

[49] J. Heidemann, W. Ye, J. Wills, A. Syed, and Y. Li, "Research Challenges and Applications for Underwater Sensor Networking," Proceedings of IEEE Wireless Communications and Networking Conference, 2006.

[50] P. Soontornpipit, C. M. Furse, Y. C. Chung, and B. M. Lin, "Optimization of a Buried Microstrip Antenna for Simultaneous Communication and Sensing of Soil Moisture," IEEE Transactions on Antennas and Propagation, vol. 54, no. 3, pp.797-800, Mar. 2006.

[51] J. Kim and Y. Rahmat-Samii, "Implanted Antenna inside a Human Body: Simulations, Designs and Characterizations," IEEE Transactions on Microwave Theory and Techniques, vol. 52, no. 8, pp.1934-1943, Aug. 2004. 
[52] D. Wang and W. Liao, "Wireless Transmission for Health Monitoring of Large Structures," IEEE Transactions on Instrumentation and Measurement, vol. 55, no. 3, pp. 972-981, Jun. 2006.

[53] N. Xu, S. Rangwala, K. K. Chintalapudi, D. Ganesan, A. Broad, R. Govindan, and D. Estrin, "A Wireless Sensor Network for Structural Monitoring," Proceedings of the 2nd International Conference on Embedded Networked Sensor Systems, Nov. 2004.

[54] G. Jolly and M. Younis,"An Energy-efficient, Scalable and Collision Less MAC Layer Protocol for Wireless Sensor Networks," Wireless Communications and Mobile Computing, vol. 5, no. 3, pp. 285-304, 2005.

[55] B. A. Warneke, M. D. Scott, B. S. Zhou, and K. S. J. Pister, "An Autonomous 16 $\mathrm{mm}^{3}$ Solar-Powered Node for Distributed Wireless Sensor Networks," Proceedings of IEEE Sensors, vol. 2, pp. 1510-1515, 2002.

[56] M. Stordeur and I. Stark, "Low Power Thermoelectric Generator - Self-Sufficient Energy Supply for Micro Systems," 16th International Conference on Thermoelectrics, pp. 575 - 577, 1997.

[57] H. Glosch, M. Ashauer, U. Pfeiffer, and W. Lang, "A Thermoelectric Converter for Energy Supply," Sensors and Actuators, vol. 74, pp. 246-250, 1999.

[58] N. S. Shenck and J. A. Paradiso, "Energy Scavenging with Shoe-Mounted Piezoelectric, " IEEE Micro, vol. 21, pp. 30-41, 2001.

[59] A. C. Patel, M. P. Vaghela, H. Bajwa, and P. K. Patra, "Power Harvesting for Low Power Wireless Sensor Network," Loughborough Antenna and Propagation Conference, pp. 633-635, 2009.

[60] P. Scholz, C. Reinhold, J. Werner, and U. Hilleringmann, "Analysis of Energy Transmission for Inductive Coupled RFID Tags," IEEE International Conference on RFID, Mar. 2007. 
[61] A. Karalis, R. E. Hamam, J. D. Joannopoulos and M. Soljacic, "Wireless Energy Transfer, including Interference Enhancement," WIPO patent WO/2009/140506, November, 2009.

[62] T. Le, K. Mayaram, and T. Fiez, "Efficient Far-Field Radio Frequency Energy Harvesting for Passively Powered Sensor Networks," IEEE Journal of Solid State Circuits, vol. 43, no. 5, pp. 1287-1302, May 2008.

[63] P. Soontornpipit, C. M. Furse, and C. C. You, "Design of Implantable Microstrip Antenna for Communication with Medical Implants," IEEE Transactions on Microwave Theory and Techniques, vol. 52, no. 8, pp. 1944-1951, Aug. 2004.

[64] EmbedSence, Wireless Sensor, Microstrain: http://www.microstrain.com/embedSense.aspx.

[65] M. M. Andringa, D. P. Neikirk, N. P. Dickerson, and S. L. Wood, "Unpowered Wireless Corrosion Sensor for Steel Reinforced Concrete," IEEE Sensors, pp. 155-158. Nov. 2005.

[66] P. Merlino and A. Abramo, "An Integrated Sensing/Communication Architecture for Structural Health Monitoring," IEEE Sensors Journal, vol. 9, no. 11, pp. 13971404, Nov. 2009.

[67] D. Mascarenas, E. Flynn, and M. Todd, "Wireless Sensor Technologies for Monitoring Civil Structures," Sound and Vibration, 2008.

[68] K. M. Farinhold, G. Park, and C. R. Farrar, "RF Energy Transmission for a LowPower Wireless Impedance Sensor Node," IEEE Sensors Journal, vol. 9, no. 7, pp.793-800, Jul. 2009.

[69] K. M. Z. Shams and M. Ali, "Wireless Power Transmission to a Buried Sensor in Concrete, "IEEE Sensors Journal, vol. 7, no. 12, pp. 1573-1577, Dec. 2007. 
[70] K. M. Z. Shams, M. Ali, and A. M. Miah, " Characteristics of an Embedded Microstrip Patch Antenna for Wireless Infrastructure Health Monitoring," IEEE Antennas and Propagation Society International Symposium, Jul. 2006.

[71] X. Jin and M. Ali, "Reflection and Transmission Properties of Embedded Dipoles and PIFAs inside Concrete at $915 \mathrm{MHz}, "$ IEEE Antennas and Propagation Society International Symposium, Jun. 2009.

[72] P. Si, A. P. Hu, S. Malpas and D. Budgett, "A Frequency Control Method for Regulating Wireless Power to Implantable Devices," IEEE Transactions on Biomedical Circuits and Systems, Vol. 2, No. 1, pp. 22-28, March 2008.

[73] S. J. A. Majerus, P. C. Fletter, M. S. Damaser and S. L. Garverick, "Low-Power Wireless Micromanometer System for Acute and Chronic Bladder-Pressure Monitoring," IEEE Transactions on Biomedical Engineering, Vol. 58, No. 3, pp.763-766, March 2011.

[74] Y. K. Song, "Active Microelectonic Neurosensor Arrays for Implantable Brain Communication Interfaces," IEEE transactions on Neural Systems and Rehabilitation Engineering, Vol. 17, No. 4, pp.339-345, August 2009.

[75] M. W. Baker and R. Sarpeshkar, "Feedback Analysis and Design of RF Power Links for Low-power Bionic Systems," IEEE Transactions on Biomedical Circuits and Systems, Vol. 1, No. 1, pp. 28-38, March 2007.

[76] M. Ghovanloo and K. Najafi, "A Wireless Implantable Multichannel Microstimulating System-on-a-chip with Modular Architecture," IEEE Transactions on Neural System and Rehabilitation Engineering, Vol. 15, No. 3, pp. 449-457, September 2007.

[77] M, Kiani and M. Ghovanloo, "An RFID-Based Closed-Loop Wireless Power Transmission System for Biomedical Applications," IEEE Transactions on Circuits and Systems-II: EXPRESS BRIEFS, Vol. 57, No.4, pp. 260-265, April 2010. 
[78] A. Ramrakhyani, S. Mirabbasi and M. Chiao, "Design and Optimization of Resonance-Based Efficient Wireless Power Delivery Systems for Biomedical Implants," IEEE Transactions on Biomedical Circuits and Systems, Vol. 5, No. 1, pp. 48-63, February 2011.

[79] G. Wang, W. Liu, M. Sivaprakasam, M. Zhou, J.D. Weiland and M. S. Humayun, "A Dual Band Wireless Power and Data Telemetry for Retinal Prosthesis," in Proceedings of IEEE EMBS Conference, pp. 28-38, August 2006.

[80] M. Catrysse, B. Hermans and R. Puers, "An Inductive Power System with Intefrated Bidirectional Data Transmision," in Proceedings of XVII Sensors, pp. 843-846, September 2003.

[81] R. Harrison, "Designing Efficient Inductive Power Links for Implantable Devices," in Proceedings of ISCAS, pp. 2080-2083, 2007.

[82] G. Yan, D. Ye, P. Zan, K. Wang and G. Ma, "Micro-robot for Endoscope based on Wireless Power Transfer," in Proceedings IEEE International Conference on Mechatronics Automation, pp. 3577-3581, August 2007.

[83] U. M. Jow and M. Ghovanloo, "Design and Optimization of Printed Spiral Coils for Efficient Transcutaneous Inductive Power Transmission," IEEE Transactions on Biomedical Circuits Systems, Vol. 1, No. 3, pp. 192-202, September 2007.

[84] A. N. Laskovski, M. R. Yuce and T. Dissanayake, "Stacked Spirals for Biosensor Telemetry," IEEE Sensors Journal, Vol. 11, No. 6, pp. 1484-1490, June 2011.

[85] S. Kim, R. R. Harrison and F. Solzbacher, "Influence of System Integration and Packaging on Its Inductive Power Link for and Integrated Wireless Neural Interface," IEEE Transactions on Biomedical Engineering, Vol. 56, No. 12, pp. 2927-2935, December 2009.

[86] O. A. Mohammed and L. F. Garcia, "A Finite Element/ Superposition Technique for the Design of Electromagnetically Coupled Coils," IEEE Transactions on Magnetics, Vol. MAG-25, No. 6, pp. 3575 - 3577, September 1989. 
[87] F. Zhang, X. Liu, S. A. Hackworth, R. J. Sclabassi and M. Sun, "In Vitro and In Vivo Studies on Wireless Powering of Medical Sensors and Implantable Devices,"IEEE/NIH Life Science Systems and Applications Workshop, pp.84-87, 2009.

[88] S. Senjuti, K. Fricke, A. Dounavis, R. Sobot, "Misalignment analysis of resonance-based wireless power transfer to biomedical implants," 2012 25th IEEE Canadian Conference on Electrical \& Computer Engineering (CCECE), vol., no., pp.1-5, April 29 2012-May 22012.

[89] A. Khripkov, Wonbin Hong, K. Pavlov, "Integrated Resonant Structure for Simultaneous Wireless Power Transfer and Data Telemetry," IEEE Antennas and Wireless Propagation Letters, vol.11, no., pp.1659-1662, 2012.

[90] A. S. Y. Poon, S. O’Driscoll and T. H. Meng, " Optimal Frequency for Wireless Power Transmission into Dispersive Tissue," IEEE Transactions on Antennas and Propagation, Vol. 55, No. 5, pp. 479-483, May 2008.

[91] M. R. Tofighi, "Characterization of Biomedical Antennas for Microwave Heating, Radiometry, and Implant Communication Applications," IEEE Wireless and Microwave Technology Conference, 2011.

[92] K. Finkenzeller, RFID Handbook: Fundamentals and Applications in Contactless Smart Cards and Identification, 2nd ed. New York:Wiley, 2003, pp. 65-112.

[93] G. Vandevoorde, and R. Puers, "Wireless energy transfer for standalone systems: a comparison between low and high energy applicability," Elsevier, Sensors and Actuators A: Physical, vol. 92, Issues 1-3, pp. 305-311, August 2001.

[94] C.A. Balanis, Antenna Theory: Analysis and Design, Wiley, New Jersey, 2005, ch. 5 .

[95] S. J. Mazlouman, A. Mahanfar, and B. Kaminska, "Mid-range wireless energy transfer using inductive resonance for wireless sensors", Proceedings of the IEEE 
international conference on Computer design. IEEE Press, Piscataway, NJ, USA, pp. 517-522, 2009.

[96] Olutola Jonah and S. V. Georgakopoulos, "Efficient wireless powering of sensors embedded in concrete via magnetic resonance," IEEE International Symposium on Antennas and Propagation (APS), vol., no., pp.1425-1428, 3-8 July 2011.

[97] R. Lundin, "A Handbook formula for the inductance of a single-layer circular coil," Proc. IEEE, vol. 73, no. 9, pp. 1428-1429, Sep. 1985.

[98] D. Fang, Handbook of Electrical Calculations. Shandong Science and Technology Press, 1994.

[99] John L. Volakis, Antenna Engineering Handbook, Fourth Edition, McGraw-Hill Professional, New Jersey, 2007, ch. 5.

[100] A. K. RamRakhyani, S. Mirabbasi,.; Chiao Mu, "Design and Optimization of Resonance-Based Efficient Wireless Power Delivery Systems for Biomedical Implants," IEEE Transactions on Biomedical Circuits and Systems, vol.5, no.1, pp.48-63, Feb. 2011.

[101] A. Karalis, Andre Kurs, Robert Moffat, D. Joannopoulos, P.H. Fisher and M. Soljacic, "Wireless energy transfer," US Patent 20110193419A1, August. 2011.

[102] Olutola Jonah, Stavros V. Georgakopoulos, "Optimal helices for wireless power transfer via magnetic resonance," 2012 IEEE 13th Annual Wireless and Microwave Technology Conference (WAMICON), , vol., no., pp.1-4, 15-17 April 2012.

[103] G. Grandi, M.K Kazimierczuk, A. Massarini, U. Reggiani, "Stray capacitances of single-layer solenoid air-core inductors,", IEEE Transactions on Industry Applications, vol.35, no.5, pp.1162-1168, Sep/Oct 1999. 
[104] S. S. Mohan, M. M. Hershenson, S. P. Boyd, and T.H. Lee, "Simple Accurate Expressions for Planar Spiral Inductances", IEEE Journal of Solid-state Circuits, Vol. 34, No. 10 (1999).

[105] G. Smith (1971, December 5); "The Proximity Effect In Systems Of Parallel Conductors And Electrically Small Multi-turn Loop Antennas" [Online]. Available: http://www.dtic.mil/cgi-bin/GetTRDoc?AD=AD0 736984.

[106] Uei-Ming Jow; M.Ghovanloo, "Design and Optimization of Printed Spiral Coils for Efficient Transcutaneous Inductive Power Transmission," , IEEE Transactions on Biomedical Circuits and Systems, vol.1, no.3, pp.193-202, Sept. 2007.

[107] Ming Wang "Embedded Strain Sensor with Power Scavenging From Bridge Vibration". Master Thesis, Dept. Civil Eng., Univ. of Maryland, College Park, MD, 2004.

[108] B. Carkhhuff and R. Cain, "Corrosion Sensors for Concrete Bridges," IEEE Instrumentation and Measurement Magazine, pp. 19-24, Jun. 2003.

[109] E G. Park, T. Rosing, M. D. Todd, C. R. Farrar, W. Hodgkiss, "Energy Harvesting for Structural Health Monitoring Sensor Network ", Journal of Infrastructure Systems, Vol. 14, pp. 64-80, 2008.

[110] D. Watters, P. Jayaweera, A. Bahr, and D. Huestis, "Design and Performance of Wireless Sensors for Structural Health Monitoring" Available: http://www2.dot.ca.gov/newtech/maintenance/docs/qnde.pdf.

[111] Xiaohua Jin; M. Ali, "Reflection and transmission properties of embedded dipoles and PIFAs inside Concrete at $915 \mathrm{MHz}, "$ Antennas and Propagation Society International Symposium, 2009. IEEE, pp.1-4, June 2009.

[112] E. G. Straser, and A. S. Kiremidjian, "A Modular, Wireless Damage Monitoring System for Structures", Report No. 128, John A. Blume Earthquake Engineering Center, Department of Civil and Environmental Engineering, Stanford University, Stanford, CA, 1998. 
[113] L. Sandrolini, U. Reggiani, and A. Ogunsola, "Modeling the electrical properties of concrete for shielding effectiveness prediction", Journal of Physics. D: Applied Physics. vol. 40, pp. 5366-5372, 2007.

[114] S.V. Georgakopoulos and Shan Jiang, "Wireless powering of sensors embedded in concrete," 2010 IEEE 11th Annual Wireless and Microwave Technology Conference, pp.1-5, 12-13, April 2010.

[115] D. Pena, R. Feick, H. Hristov, and W. Grote, "Measurement and Modeling of Propagation Losses in Brick and Concrete Walls for the 900-MHz Band," IEEE Transaction on Antennas and Propagation, vol. 51, no. 1, pp. 31-39, Jan. 2003.

[116] E. Richalot, M. Bonilla, M. Wong, V. Fouad-Hanna, H. Baudrand, and J. Wiart, "Electromagnetic Propagation into Reinforced-Concrete Walls, "IEEE Transactions on Microwave Theory and Techniques, vol. 48, no. 3, pp. 357-366, Mar. 2000.

[117] D . Poljak, Human Exposure to Electromagnetic Fields, WIT Press, LLC, ISBN 1-85312-997-6, 2004.

[118] Vitaliy Zhurbenko, Advanced Microwave Circuits and Systems, ch 19, InTech publisher, 2010.

[119] D.C Ng, Xiaoting Wang, G.K.Felic, S. Bai,; C. S. Boyd, M. Halpern, E. Skafidas, "Specific absorption rate distribution on a human head model from inductive power coils," EMC Europe 2011 York, vol., no., pp.79-83, 26-30 Sept. 2011.

[120] S. Georgakopoulos, and E. Tentzeris, "Highly Efficient Wireless Power Transfer Through Embedded Geometric Configurations" USPTO Provisional patent number 61/658,596, 2012.

[121] E. Sardini, M. Serpelloni, Instrumented "wearable belt for wireless health monitoring", Procedia Engineering, Volume 5, 2010, Pages 580-583, ISSN 1877 7058, 10.1016/j.proeng.2010.09.176 
[122] Chee Kian Lim, Zhiqiang Luo, I-Ming Chen, Song Huat Yeo, "Wearable wireless sensing system for capturing human arm motion", Sensors and Actuators A: Physical, Volume 166, Issue 1, March 2011, Pages 125-132, ISSN 0924-4247, 10.1016/j.sna.2010.10.015.

[123] P. S. Pandian, K. Mohanavelu, K. P. Safeer, T. M. Kotresh, D. T. Shakunthala, Parvati Gopal, V. C. Padaki, "Smart Vest: Wearable multi-parameter remote physiological monitoring system", Medical Engineering \& Physics, Volume 30, Issue 4, May 2008, Pages 466-477.

[124] Paul F. Baude, David A. Ender, Tommie W. Kelley, "Organic Semiconductor RFID Transponders", IEEE IEDM Conf., 2003, vol. 3, pp. 191-194.

[125] Amin Shameli, Aminghasem Safarian, Franco De Flaviis, Ahmadreza Rofougaran, "An RFID System with Fully Integrated Transponder", IEEE Radio Frequency Integrated Circuits Symposium, 2007, pp. 285-288.

[126] His-Tseng Chou, Chia Tung, Tso-Ming Hung, "Design of a Near-Field Focused Reflectarray Antenna for RFID Reader Applications", IEEE Antennas and Propagation Society International Symposium, 2010.

[127] Xiuping Li and Zijiang Yang, "Dual-Printed-Dipoles Reader Antenna for UHF Near-Field RFID Applications", IEEE Antennas and Wireless Propagation Letters, 2011, vol. 10, pp. 239-242.

[128] Zi Long Ma, Li Jun Jiang and Jingtian Xi, "A Single Layer Compact HF-UHF Dual Band RFID Tag Antenna", IEEE Antennas and Wireless Propagation Letters, 2011, vol. 11, pp. 1257-1260. 
VITA

\section{OLUTOLA JONAH}

1974

Born, Ilesha, Nigeria

2000 B. Sc., Electrical and Electronic Enginnerring Obafemi Awolowo University

Ile-Ife, Nigeria

2008

M. Sc., Electrical and Electronic Enginnerring

Obafemi Awolowo University

Ile-Ife, Nigeria

2013

Ph. D. Candidate, Electrical Engineering

Florida International University

Miami, USA

\section{PUBLICATIONS AND PRESENTATIONS}

JOURNALS

[1] Olutola Jonah and Stavros V. Georgakopoulos, "Wireless Power Transfer in Concrete via Strongly Coupled Magnetic Resonance", IEEE Transactions on Antennas and Propagation, Vol. 61, no. 3, pp. 1378-1384, March 2013.

[2] Olutola Jonah, Stavros V. Georgakopoulos and Manos M.Tentzeris,"Optimal Design Parameters for Wireless Power Transfer by Resonance Magnetic," IEEE Antennas and Wireless Propagation Letters, vol.11, no., pp.1390-1393, 2012.

[3] O. Jonah, A Merwaday, S. V. Georgakopoulos and Manos M. Tentzeris, "Spiral Resonators for Optimally Efficient Strongly Coupled Magnetic Resonant Systems", IEEE microwave and communication letters (to be Submitted).

[4] O. Jonah, S. V. Georgakopoulos and Manos M. Tentzeris, " Conformal Strongly Coupled Wireless Powering in Biomedical Devices, IEEE microwave and communication letters (to be Submitted).

[5] O. Jonah, S. V. Georgakopoulos and Manos M. Tentzeris, "Efficient Misalignment Insensitive Wireless Power Transfer via Magnetic Resonance", IEEE Transactions on Antennas and Propagation (to be Submitted). 
[6] O. Jonah and S. V. Georgakopoulos, "Optimal Parameters of Coupled System for Wireless Power Transfer by Resonance Magnetic", IEEE microwave and communication letters (to be Submitted).

\section{CONFERENCES}

[1] Olutola Jonah and S. V. Georgakopoulos, "Efficient wireless powering of sensors embedded in concrete via magnetic resonance," IEEE International Symposium on Antennas and Propagation (APS), 2011, vol., no., pp.1425-1428, 3-8 July 2011.

[2] Olutola Jonah and S. V. Georgakopoulos, "Wireless power transmission to sensors embedded in concrete via Magnetic Resonance," 2011 IEEE 12th Annual Wireless and Microwave Technology Conference (WAMICON), vol., no., pp.1-6, 18-19 April 2011.

[3] Stavros V. Georgakopoulos and Olutola Jonah, "Optimized wireless power transfer to RFID sensors via magnetic resonance," IEEE International Symposium on Antennas and Propagation (APS), 2011, vol., no., pp.1421-1424, 3-8 July 2011.

[4] Olutola Jonah and S. V. Georgakopoulos, "Wireless Power transfer to RFID Sensors via Magnetic resonance ", IEEE International Conference on RFID, Orlando, FL, Apr.12, 2011.

[5] Olutola Jonah and S. V. Georgakopoulos, "Wireless powering of biomedical device via magnetic resonance," 2012 IEEE 13th Annual Wireless and Microwave Technology Conference (WAMICON), vol., no., pp.1-6, 15-17 April 2012.

[6] Olutola Jonah and Stavros V. Georgakopoulos, "Optimal helices for wireless power transfer via magnetic resonance," 2012 IEEE 13th Annual Wireless and Microwave Technology Conference (WAMICON), vol., no., pp.1-4, 15-17 April 2012.

[7] Olutola Jonah and Stavros V. Georgakopoulos, "Wireless power transmission to sensors in reinforced concrete via Magnetic Resonance," 2012 IEEE Antennas and Propagation Society International Symposium (APS), vol., no., pp.1-2, 8-14 July 2012.

[8] Olutola Jonah and Stavros V. Georgakopoulos, "Specific Absorption Rate (SAR) distribution in human tissue with magnetic resonance," 2012 IEEE Antennas and Propagation Society International Symposium (APS), vol., no., pp.1-2, 8-14 July 2012.

[9] Olutola Jonah and Stavros V. Georgakopoulos" Wireless powering of device embedded in concrete via Magnetic Resonance," 2012 IEEE Antennas and Propagation Society International Symposium (APS), vol., no., pp.1-2, 8-14 July 2012. 